\title{
Multifaceted Applications of Microbial Pigments: Current Knowledge, Challenges and Future Directions for Public Health Implications
}

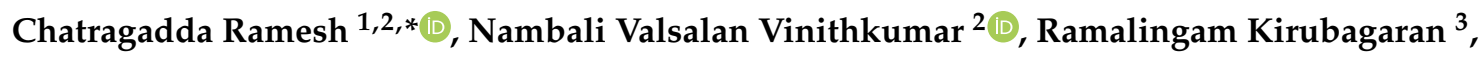 \\ Chidambaram Kulandaisamy Venil ${ }^{4}$ and Laurent Dufossé ${ }^{5, *(\mathbb{D})}$ \\ 1 National Centre for Coastal Research (NCCR), NCCR Field Office, Ministry of Earth Sciences (MoES), \\ Mandapam Camp 623519, India \\ 2 Atal Centre for Ocean Science and Technology for Islands, ESSO-NIOT, Dollygunj, Port Blair, \\ Andaman and Nicobar Islands 744103, India \\ 3 Marine Biotechnology Group, ESSO-National Institute of Ocean Technology (NIOT), \\ Ministry of Earth Sciences (Govt. of India), Chennai 600100, India \\ 4 Anna University, Department of Biotechnology, Coimbatore 641046, India \\ 5 Laboratoire de Chimie des Substances Naturelles et des Sciences des Aliments-LCSNSA EA 2212, \\ Université de La Réunion, ESIROI Agroalimentaire, 97744 Saint-Denis, France \\ * Correspondence: chrameshpu@gmail.com (C.R.); laurent.dufosse@univ-reunion.fr (L.D.); \\ Tel.: +91-(0)3192-225083/95 (C.R.); +33-262217544 (L.D.)
}

Received: 27 May 2019; Accepted: 27 June 2019; Published: 28 June 2019

\begin{abstract}
Microbial oddities such as versatile pigments are gaining more attention in current research due to their widely perceived applications as natural food colorants, textiles, antimicrobial activities, and cytotoxic activities. This indicates that the future generation will depend on microbial pigments over synthetic colorants for sustainable livelihood. Although several reviews have detailed the comprehensive applications of microbial pigments extensively, knowledge on several aspects of pigmented microbes is apparently missing and not properly reviewed anywhere. Thus, this review has been made to provide overall knowledge on biodiversity, distribution, pathogenicity, and ecological and industrial applications of microbial pigments as well as their challenges and future directions for food, industrial, and biomedical applications. Meticulously, this compendious review treatise on the pigments from bacteria, fungi, yeasts, and microalgae includes reports from the 1970s to 2018. A total of 261 pigment compounds produced by about 500 different microbial species are included, and their bioactive nature is described.
\end{abstract}

Keywords: microbial pigments; pigment compounds; food colorants; bioactive pigment molecules; pigment applications

\section{Introduction}

Microbial communities have an enormous potentiality to produce diverse and mesmerizing aesthetic traits, such as knack emission of bioluminescence and fluorescence, formation of magnetosomes, production of bioactive metabolites, and different pigments for scientific succulence. Colloquially, directly or indirectly, microbial communities play an important integrated role in the biosphere by regulating biogeochemical and ecological processes [1]. Regardless of their role in the environment, they offer several benefits to humanity; one such benefit is pigment production by several microbes, of which deserved importance is being highlighted in recent times, and there are still more untapped sources to explore many unknown pigmented compounds [2]. The importance of microbial pigments has been emphasized in different applications, such as cosmetics, food, pharmaceuticals, and 
textiles, and these compounds are also well-known to exhibit cytotoxic, antioxidant, antimicrobial, antimalarial, anticancer, antitumor, and antifouling activities [3-7].

Pigments are molecules that absorb a specific wavelength of light and reflect the rest of the pulchritude visible spectrum (380-750 nm). Pigment production is one of the charismatic traits of microbes. Apparently, microbial pigments are not merely colors, but they possess a mixture of diverse chemical components with multifaceted potential biological activities [8]. In the last two decades, studies on pigmented microorganisms from terrestrial and marine ecosystems have tremendously expanded, resulting in the use of pigments in cancer-related research.

Microbial pigmented molecules such as bacteriochlorophylls, carotenoids, flavins, indigoids, melanins, pheomelanin, monascins, phenazines, phenazostatin $\mathrm{D}$, prodigiosin, quinone precursors, violacein, glaukothalin, pycocyanin, xanthomonadin, phenazine, canthaxanthin, astaxanthin, $\beta$-carotene, etc. are produced as biproducts by several microorganisms [3,9-12]. Many of these compounds and their derivatives are reported to show wide range of cell specific biological activities which are expressed in effective/inhibitory/lethal/subleathal concentrations such as effective dose (ED), growth inhibitory concentration (GIC), minimum inhibitory concentration (MIC), Half maximal effective concentration $\left(\mathrm{EC}_{50}\right)$, half maximal growth inhibition $\left(\mathrm{GI}_{50}\right)$, half maximal inhibitory concentration $\left(\mathrm{IC}_{50}\right)$, half maximal lethal concentration $\left(\mathrm{LC}_{50}\right)$, half maximal lethal dose $\left(\mathrm{LD}_{50}\right)$, and tumour growth inhibition of $50 \%$ (TGI50) [13].

There are several synthetic colorants that are being developed as immunosuppressive and anticancer drugs [14]. Historical notes on several synthetic dyes and pigments are of industrial applications (textile, cosmetics, and food), and their disadvantages have recently been well-detailed [15, 16]. Since a number of synthetic pigments and their biproducts are found to display toxic, teratogenic, and carcinogenic properties, the exploration of natural pigments from microbes has emerged in recent years due to their biodegradability than synthetic counterparts [17]. Several natural pigment compounds originated from different sources are being used as food colorants [18]. Irrespective of plant, animal, and synthetic pigments, microbial pigments are much mellower and highly preferred due to their higher productivity and optimizable culture conditions. Pigment productions by Monascus, Rhodotorula, marine actinomycetes, marine Pseudoalteromonas, and marine cyanobacterial species have been widely studied. In this context, this review appends a plethora of industrially important pigmented molecules produced by different microorganisms such as bacteria, fungi, cyanobacteria, and yeast.

\section{Microbial Pigments and Chemical Structures}

Microbial pigments are usually seen in two forms as pigments diffused out into the media and pigments retained within the cells. To produce any kind of pigmented compound with potential biological properties from any organism for industrial applications, the following requirements need to be fulfilled: Organisms should be amenable to culture, have a fast growth rate, be optimizable, have a high productivity in limited space and in short time, be available and able to produce throughout the year, be nontoxigenic, be nonpathogenic, be able to grow in a wide range of nutrients such as carbon and nitrogen sources, and be tolerant to a broad spectrum of physical (light and temperature) and chemical ( $\mathrm{pH}$ and osmolarity) parameters used in production. Mostly, these requirements are met with microbes; hence, many research studies have been prioritized and focused on microbes (particularly marine bacteria are highly preferred due to their higher productivity and optimizable culture conditions) as potential sources over plants, animals, and synthetic compounds. Further inclination towards optimization and improvement of mass production of pigments are encouraged with genetic engineering as well.

There are four main sources of pigments for various applications as mentioned above: (1) Plant-derived pigments, (2) animal-derived pigments, (3) microbial pigments, and (4) synthetic pigments. Here, merely microbial pigments are reviewed due to their insatiable demand over the rest of the sources and for better understanding on the same aspect. Some of the carotenoid pigments, 
such as acetylenic carotenoids appear to be restricted to certain environments, e.g., some marine bacteria Planococcus maritimus and Rubritalea squalenifaciens biosynthesize acyclic $\mathrm{C}_{30}$-type carotenoic acids [19]. In nature, $\mathrm{C}_{50}$ Carotenoids such as sarcinaxanthin and decaprenoxanthin are exclusively biosynthesized by Halobacteria, Halococcus, Actinomycetales, Flavobacterium dehydrogenans, Arthrobacter sp., Micrococcus luteus, Dietzia sp., Corynebacterium poinsettiae, C. glutamicum, and a strain of Pseudomonas, and these carotenoids appear to be not produced by plants [20,21]. Similarly, aryl carotenoids such as isorenieratene, 3-hydroxy-isorenieratene and 3,3'-di-hydroxy-isorenieratene are found in very few microorganisms, such as Brevibacterium linens, Streptomyces mediolani, and Mycobacterium aurum [21]. Identifying metabolic pathways and genes responsible for such rare phenomena are of great importance for genetic engineering studies to develop rare carotenoids with therapeutic application.

The chemical structures of some of the major pigment molecules are given below: carotenoids: Acyl glyco-carotenoic acid (diapolycopenedioic acid) (1), adonixanthin (2), alloxanthin (3), anhydrorhodovibrin (4), antheraxanthin (5), astaxanthin (6), aleuriaxanthin (7), aphanicin (8), aphanizophyll (9), auroxanthin (10), $\beta$-carotene (11), bacterioruberin (12), caloxanthin (13), canthaxanthin (14), chlorobactene (15), chloroxanthin (16), crocoxanthin (17), cryptoxanthin (18), deinoxanthin (19), decaprenoxanthin (20), demethylspheroidene (21), demethylspheroidenone (22), diadinoxanthin (23), diatoxanthin (24), diapolycopene (25), dinoxanthin (26), echinenone (27), ergoxanthin (28), escholtzxanthin (29), eutreptiellanone (30), flavacin (31), flexirubin (32), flexixanthin (33), fucoxanthin (34), gyroxanthin diester (35), heteroxanthin (36), isorenieratene (37), lutein (38), loroxanthin (39), lycopene (40), monadoxanthin (41), mutachrome (42), mutatoxanthin (43), mycosporine (44), myxobactin (45), myxobactone (46), keto-myxocoxanthin (47), myxoxanthophyll (48), Nanocystis exedens pigments (49), nostoxanthin (50), neoxanthin (51), neurosporene (52), okenone (53), oscilloxanthin (54), phoenicoxanthin (55), phytoene (56), prasinoxanthin (57), pyrroxanthin (58), rhodopin (59), rhodovibrin (60), salinixanthin (61), saproxanthin (62), sarcinaxanthin (63), siphonein (64), siphonaxanthin (65), spheroidene (66), spheroidenone (67), spirilloxanthin (68), staphyloxanthin (69), torulene (70), torularhodin (71), vaucherixanthin (72), violaxanthin (73), vioxanthin (74), xanthomonadines (75), xanthophyll (76), and zeaxanthin (77); phycobiliproteins: cyanophycin (78), phycocyanin (79), phycocyanobilin (80), phycoerythrin (81), phycoerythrobilin (82), and phycourobilin (83); flavins: ankaflavin (84), monascoflavin (85), riboflavin (86), roseoflavin (87), and toxoflavin (88); melanins: melanin precursors such as catechol (89), 1,8-dihydroxynaphthalene (DHN) (90), Dopa (91), eumelanin (92), L-glutaminyl-4-hydroxybenzene (GHB) (93), homogentisic acid (HGA) (94), lincomycin (95), phaeomelanin (96), phenazostatin D (97), and all trans-retinal (98); heterocyclic pigments: cycloprodigiosin (99), indigotine (indigo) (100), indigoidine (101), prodigiosin (102), undecylprodigiosin (103), and violacein (104); phenazine compounds: actinomycin D (105), chlororaphine (106), Dihydrophencomycin (107), griseolutein (108), iodinin (109), myxin (cuprimycin) (110), oxychlororaphine (111), phenazine-1-carboxylic acid (112), phenozostatin D (113), pyocyanin (114), pyorubin (aeruginosin $A+B)(\mathbf{1 1 5})$, and pyoverdin (116); quinones: arpink red (117), averythrin (118), austrocortinin (119), bostrycoidin (120), catenarin (121), cercosporin (122), chlorobiumquinone (123), 7-chloroemodin (124), chrysophanol (125), citreorosein (126), cynodontin (127), dermocybin (128), dermoglaucin (129), dermorubin (130), draconin (131), elsinochromes (132), emodin (133), erythroglaucin (134), fallacinal (135), flaviolin (136), flavomannin (137), fusarubin (138), helminthosporin (139), javanicin (140), juglone (141), Karuquinone A (142), menaquinone-7 (143), naphthoquinone (144), nectriachrysone (145), pachybasin (146), parietinic acid (147), phomaligin A (148), phomarin (149), physcion (150), piloquinone (151), questin (152), rubellin D (153), rubrocristin (154), skyrin (155), spinulosin (156), teloschistin (157), tritisporin (158), and Xylindein (159); monascus pigments: ankaflavin (84), lovastatin (160), monascins (161), monascoflavin (85), monascorubramine (162), monascorubrin (163), rubropunctamine (164), and rubropunctatine (165); other compounds: acetylazulene (166), actinorhodin (167), akashin (168), albidin (169), alterperylenol (170), amitenone (171), ammosamide A (172), ammosamide B (173), atranorin (174), atromentin (175), aulosirazole (176), aurantricholide B (177), aurasperone A (178), azaphilone (179), azulene (180), boviquinone 3 (181), 
bromoalterochromides (182), calycin (183), candidin (184), chloronatronochrome (185), chrysogenin (186), citrinin (187), cochliodinol (188), cordycepin (189), cordycepoid A (190), diastaphenazine (191), dihydroalterperylenol (192), dihydroxyazulene (193), dolastatin (194), epicocconone (195), floccosin (196), fluorescein (197), fonsecin (198), glauconic acid (199), glaukothalin (200), gomphidic acid (201), granadaene (202), grevilline A (203), gyrophoric acid (204), haematopodin (205), hyaluromycin (206), hypericin (207), iridosporin (208), lactaroviolin (209), laetiporic acid A (210), lilacinone (211), luteosporin (212), magnesidin (213), marineosin A (214), marinone (215), melanocrocin (216), mitorubrin (217), mycenaaurin A (218), N-carboxamidostaurosporine (219), natronochrome (220), nicotine (221), nostocine A (222), panosialin (223), peridinin (224), pestalone (225), Phlegmacin A (226), phenoxazine (227), porphyrin (228), pulvinic acid (229), purpuride (230), pyrandione (231), pyrrocidine A (232), rhizocarpic acid (233), roseophilin (234), rubrolone (235), rubrosporin (236), rubrosulphin (237), rumbrin (238), sanguinone A (239), scytonemin (240), siroheme (241), sorbicillin (242), stearoyldeterrol (243), sterigmatocystin (244), streptochlorin (245), tambjamine (246), tetrabromopyrrole (247), thermorubin (248), tryptanthrin (249), variegatorubin (250), viomellein (251), viopurpurin (252), vulpinic acid (253), xanthomegnin (254), bacterial luciferin (255), dinoflagellate luciferin (256), blue fluorescent protein-lumazine (BFP) (257) and yellow fluorescent protein (YFP) chromophore (258); and chlorophylls: bacteriochlorophylls (259), chlorophylls (260) and divinyl-chlorophylls (261) occurring in different microorganisms are illustrated (Figure 1; Table S1). Here, we also categorize the microbial pigments into two categories: (1) Fluorescent pigments (phycoerythrins, fluorescein, epicocconone, BFP, and YFP) and (2) nonfluorescent pigments (rest of the pigmented compounds as detailed above). 
<smiles></smiles>

Diapolycopenedioic acid (1)

$$
\mathrm{C}_{49} \mathrm{H}_{70} \mathrm{O}_{9}
$$

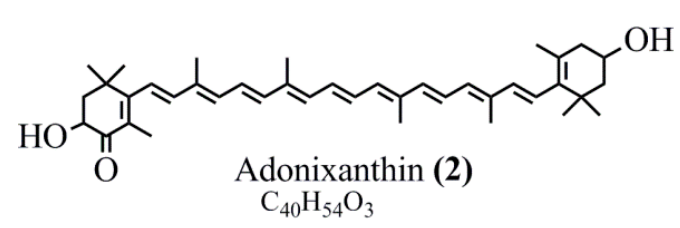<smiles>CC(C)=CC=CC(C)=CC=CC(C)=CC=CC=C(C)C=CC1=C(C)CC(O)C1(C)C</smiles>

$\mathrm{OH}_{3} \mathrm{C}$ Anhydrorhodovibrin (4) $\mathrm{C}_{41} \mathrm{H}_{58} \mathrm{O}$

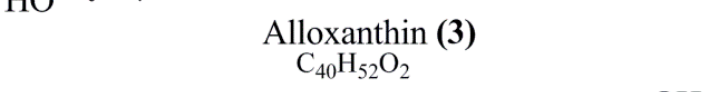

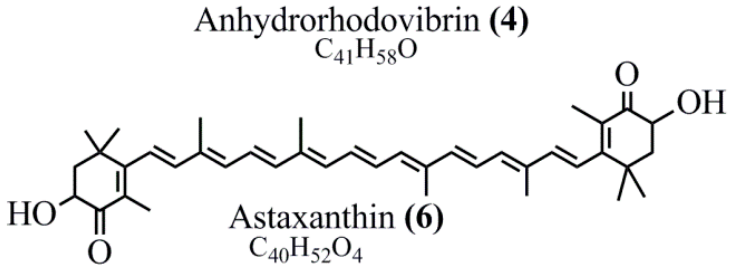

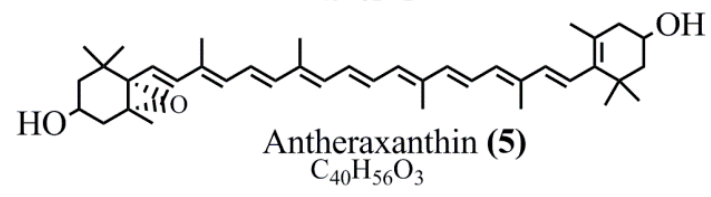
$\underset{\mathrm{C}_{40} \mathrm{H}_{56} \mathrm{O}_{3}}{\text { Antheraxanthin (5) }}$<smiles>C=C(C)C(O)CC/C=C(C)/C=C/C(C)=C/C=C/C(C)=C/C=C/C=C(C)/C=C/C=C(C)/C=C/C1=C(C)CCCC1(C)C</smiles>
$\underset{\mathrm{C}_{40} \mathrm{H}_{56} \mathrm{O}}{\text { Aleuriaxanthin (7) }}$<smiles>CC1=C(/C=C/C=C(C)/C=C/C(C)=C/C=C/C(C)=C\C=C\C(C)=C\C=C\C(C)=C\C=C\C(O)(O)C2OC(C)C(O)C(O)C2O)C(C)(C)CC(O)C1O</smiles>

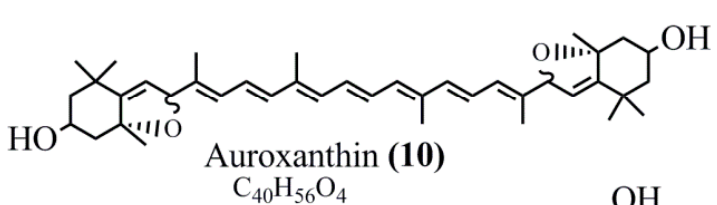

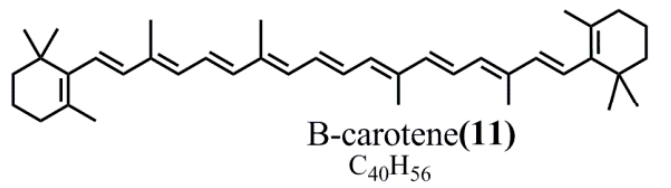

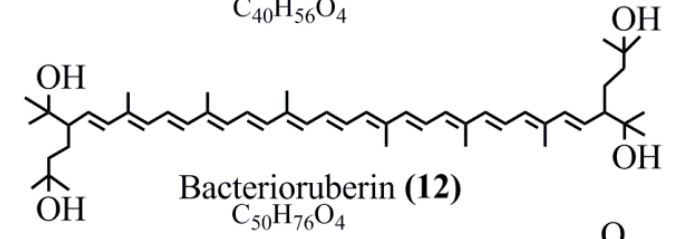<smiles>CC1=C(C)C(C)(C)C(O)CC1O</smiles>

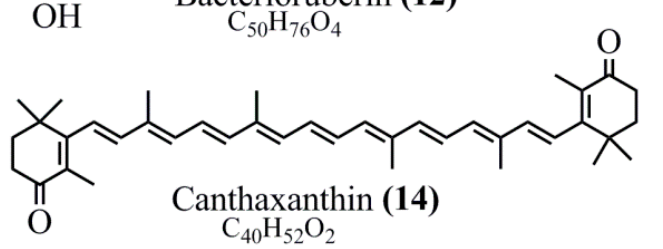

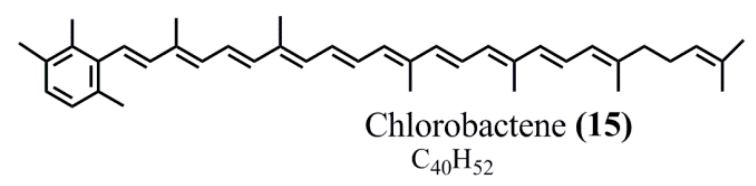

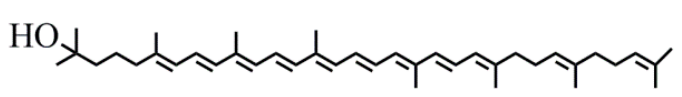

Chloroxanthin (16)

$$
\mathrm{C}_{40} \mathrm{H}_{60} \mathrm{O}
$$

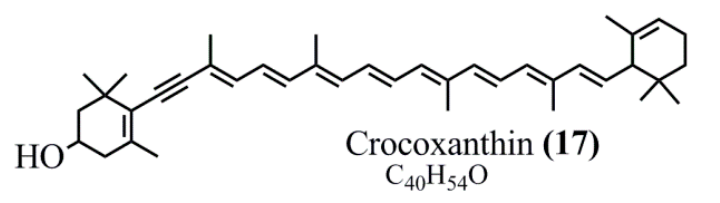

Figure 1. Cont. 


$$
\text { (18) }
$$

(2)

Demethylspheroidene (21)

Demethylspheroidenone (22)

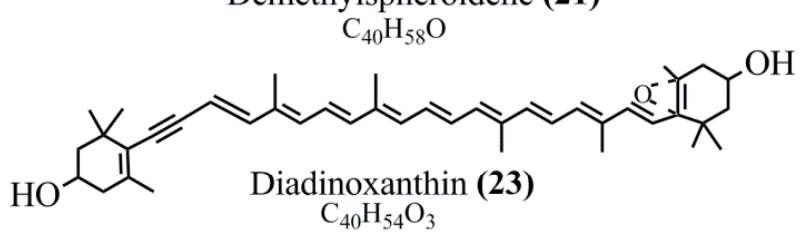

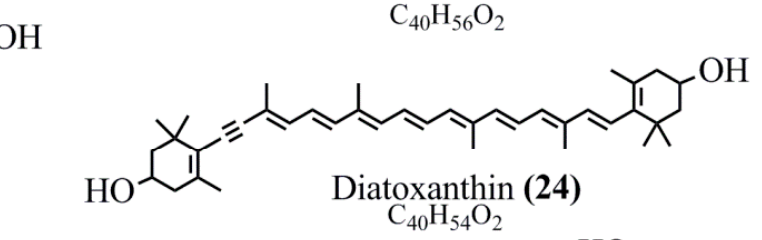<smiles>CC(C)=CC=CC=C(C)C=CC=C(C)C=CC=CCC=C(C)C=CC=C(C)C=CC=C(C)C</smiles>

Diapolycopene (25)

$$
\mathrm{C}_{30} \mathrm{H}_{40}
$$

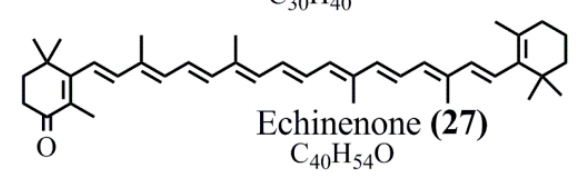

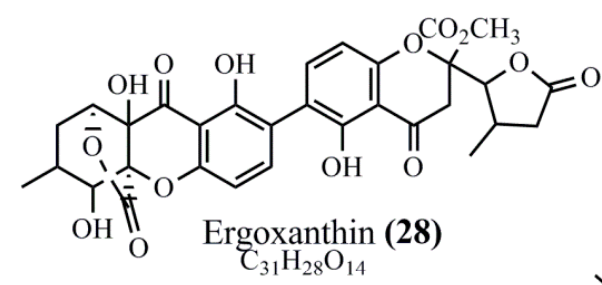

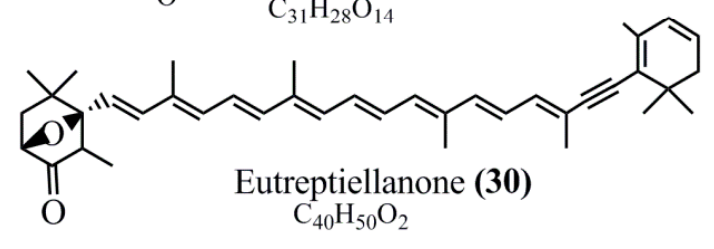

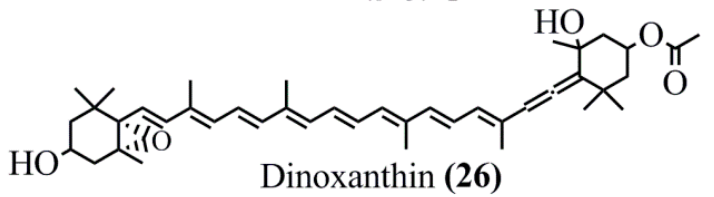
$\mathrm{C}_{42} \mathrm{H}_{58} \mathrm{O}_{5}{ }^{\circ}$

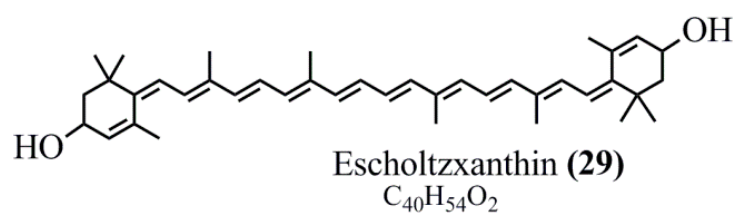

Escholtzxanthin (29)

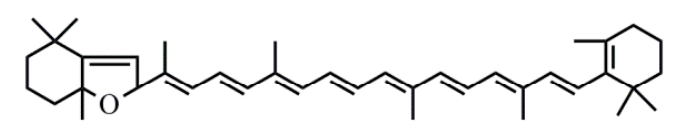

Flavacin (31)

$\mathrm{C}_{40} \mathrm{H}_{56} \mathrm{O}$

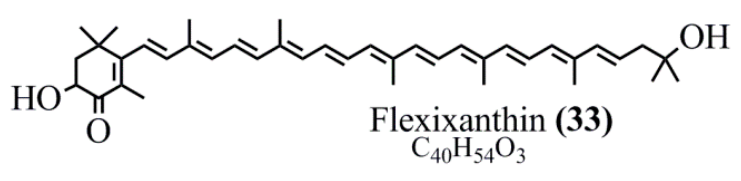

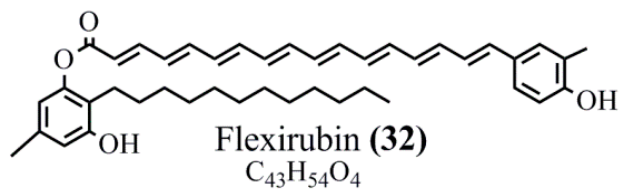
$\mathrm{C}_{40} \mathrm{H}_{54} \mathrm{O}_{3}$<smiles>[H]</smiles><smiles>C=C/C(C)=C/C=C/C=C(C)/C=C/C=C(C)/C(C)=C/C[C@@]1(O)C(C)(C)CC(O)C[C@]1(C)O</smiles>

Figure 1. Cont. 


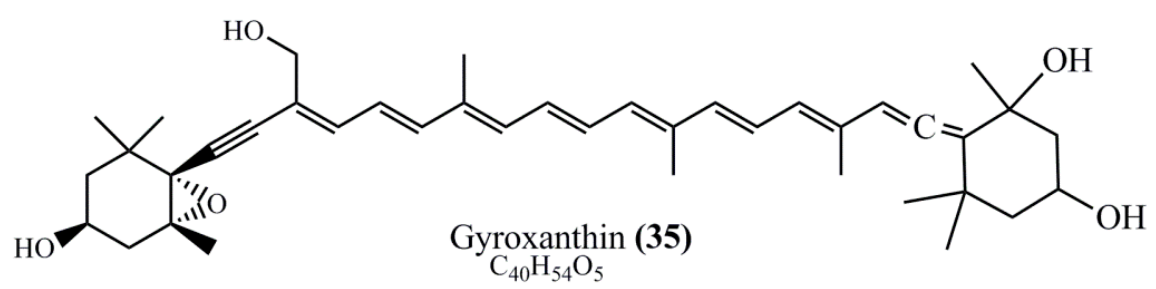

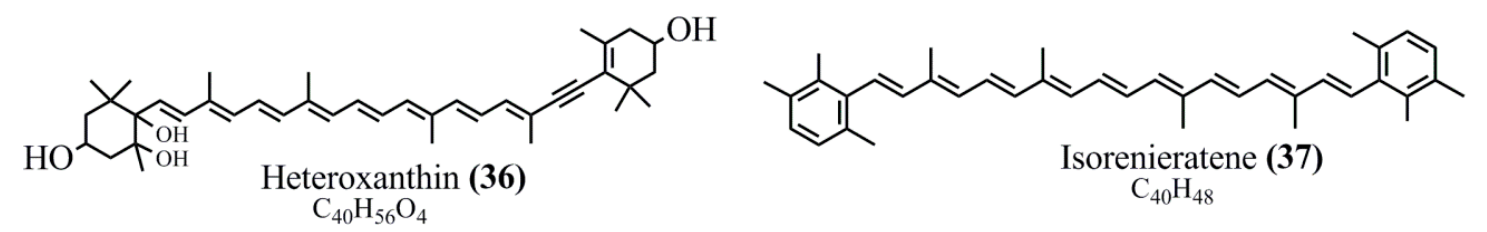

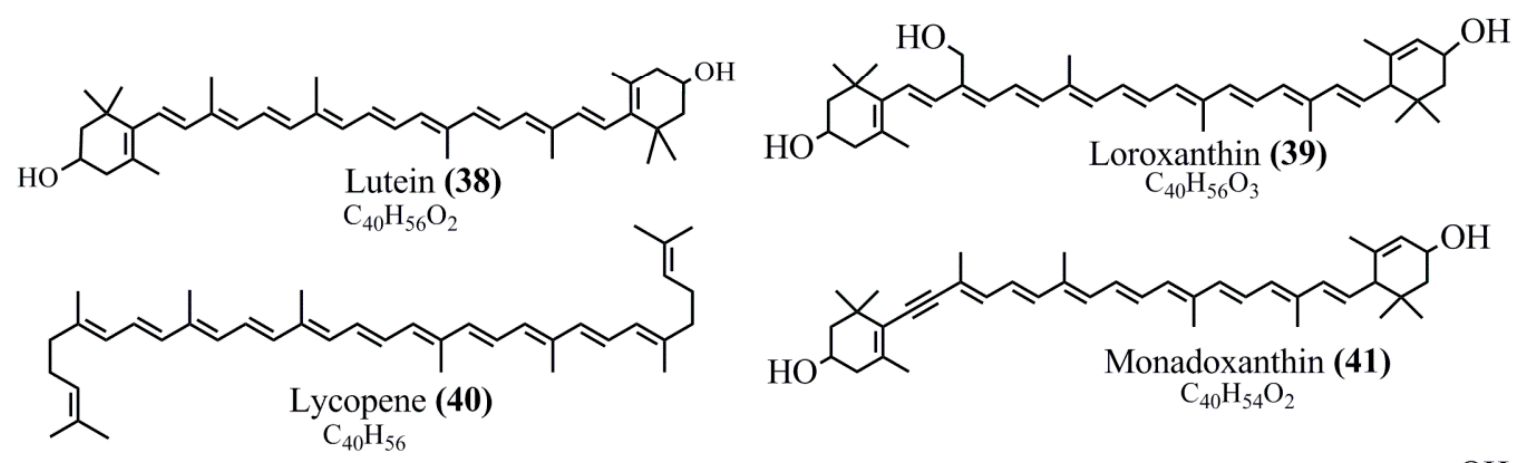

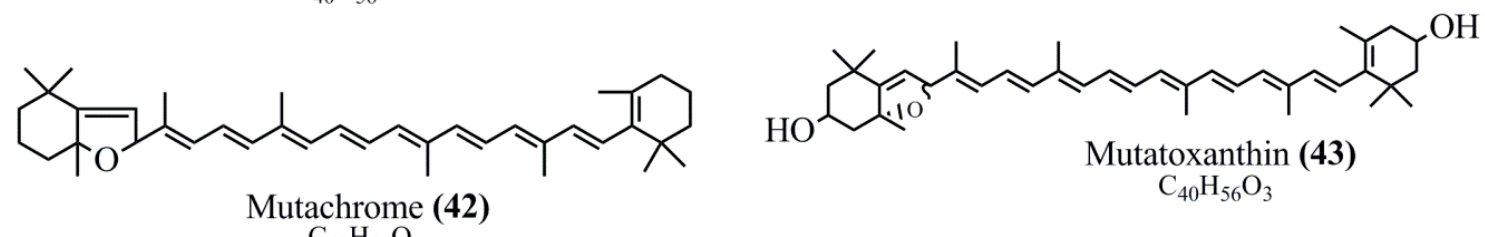

$$
\mathrm{C}_{40} \mathrm{H}_{56} \mathrm{O}
$$

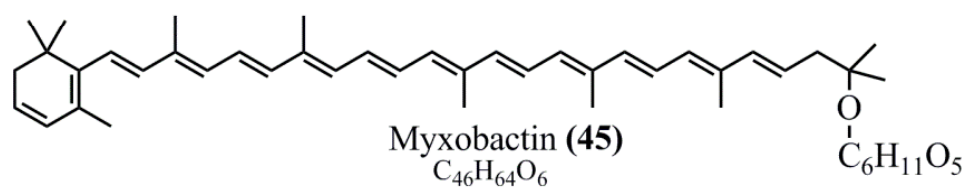<smiles>COC1=C(NC(CO)CO)CC(O)(CO)CC1=O</smiles><smiles>CCCCCOC(C)(C)C/C=C/C(C)=C/C=C/C(C)=C/C=C/C(C)=C/C=C/C=C(C)/C=C/C=C(C)/C=C/C=C1C(C)=C(C)CCC1(C)C</smiles>

Mycosporine (44) $\mathrm{C}_{11} \mathrm{H}_{19} \mathrm{NO}_{6}$

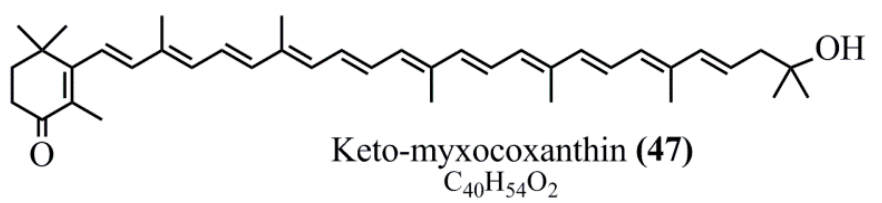

Figure 1. Cont. 


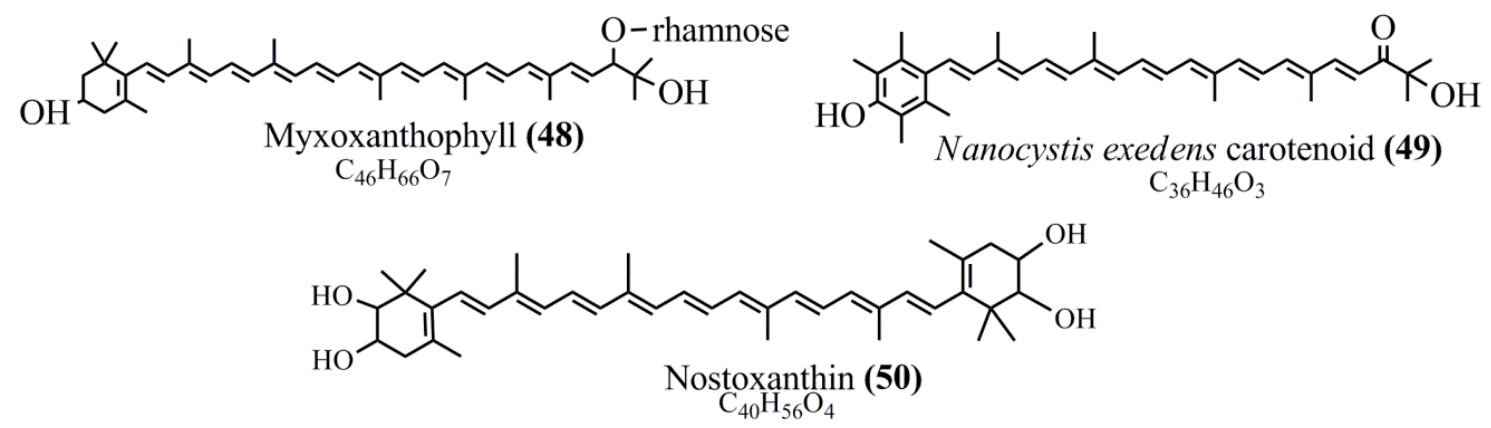

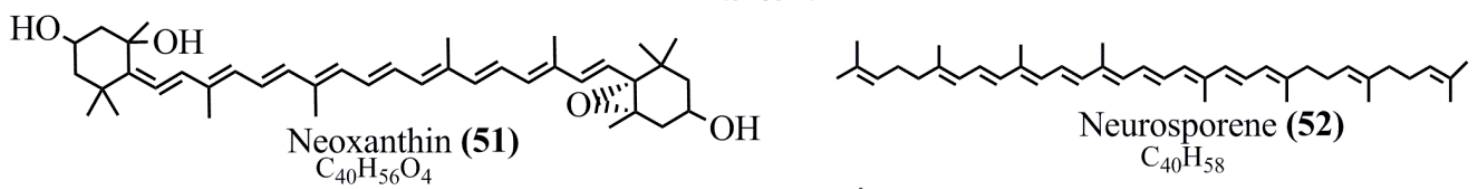

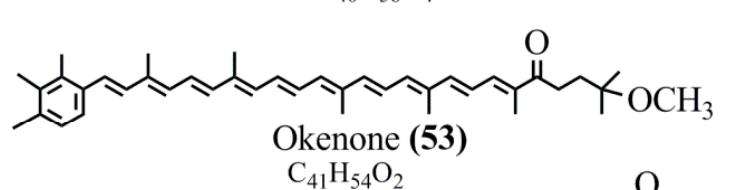

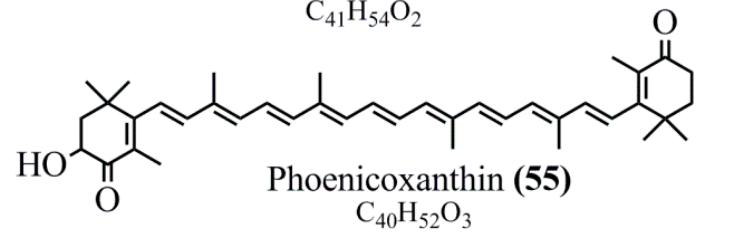

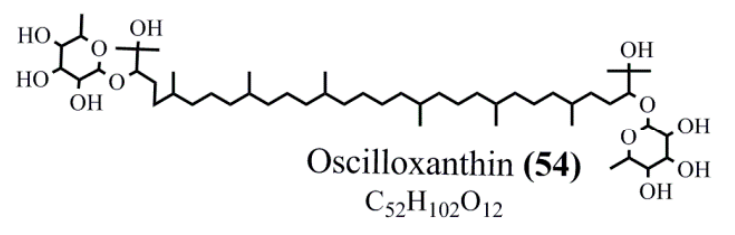

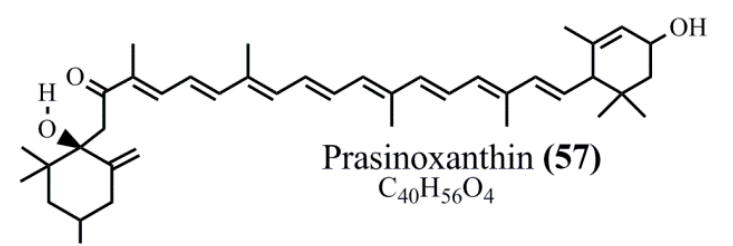<smiles>CC(C)=CCC/C(C)=C/CC/C(C)=C/CC/C(C)=C/C=C/C=C(\C)CC/C=C(\C)CC/C=C(\C)CCC=C(C)C</smiles><smiles>CC(=O)OC1CC(C)=C(C#CC(C)=CC=CC=CC=CC(C)=CC=C2C=C(C=CC3=CC(=O)CC(C)(C)C3)C(=O)O2)C(=O)O1</smiles>

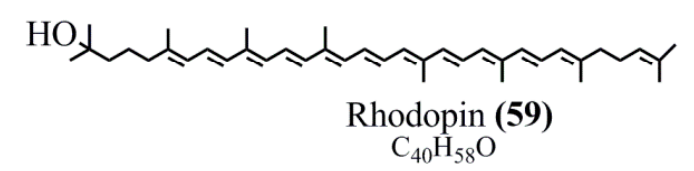

$\mathrm{OCH}_{3}$

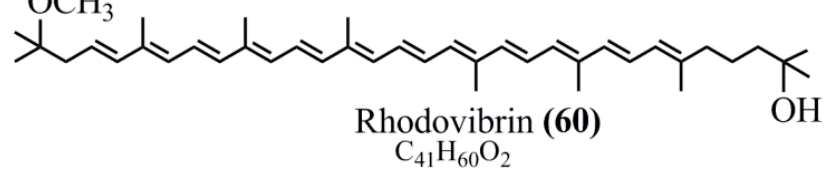<smiles>CC1=C(/C=C/C(C)=C/C=C/C(C)=C/C=C/C=C(C)/C=C/C=C(C)/C=C/C=C(C)/C=C/[C@@H](O)C(C)(C)OC2OC(COC(=O)CCCCCCCCCCCCCCC(C)C)C(O)C(O)C2O)C(C)(C)CCC1=O</smiles>

Figure 1. Cont. 


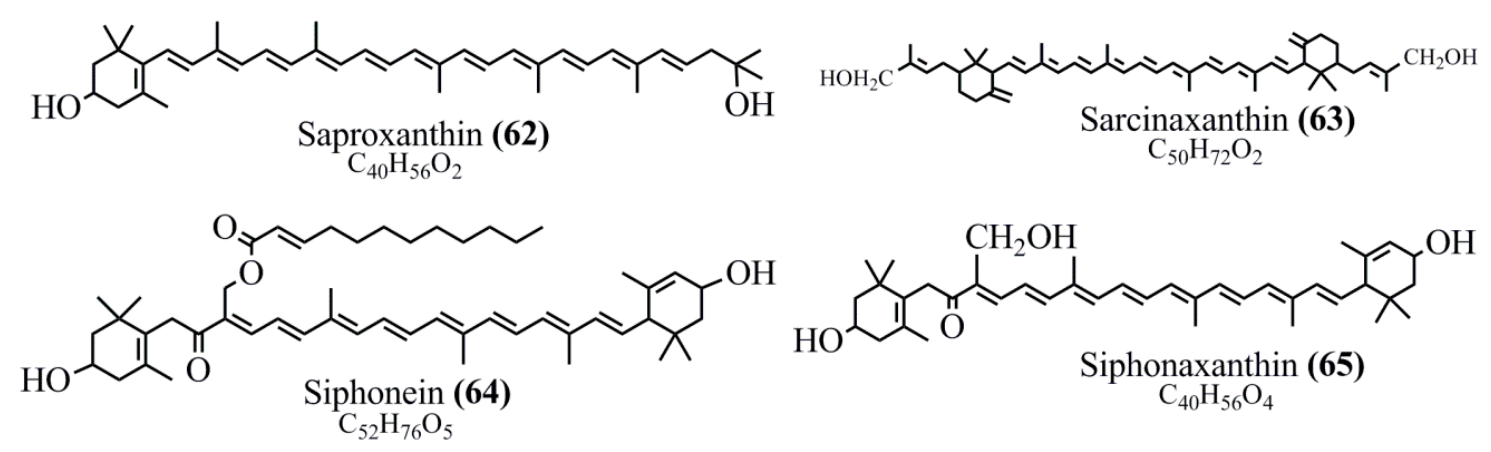

$\mathrm{OH}_{3} \mathrm{C}$

Spheroidene (66) $\mathrm{C}_{41} \mathrm{H}_{60} \mathrm{O}$

 $\underset{\mathrm{C}_{42} \mathrm{H}_{60} \mathrm{O}_{2}}{\text { Spirilloxanthin (68) }}$

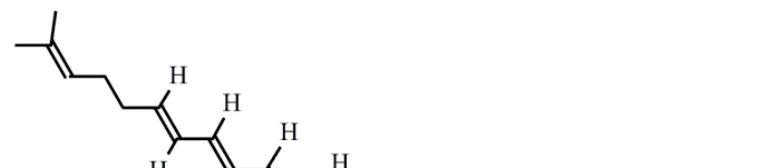

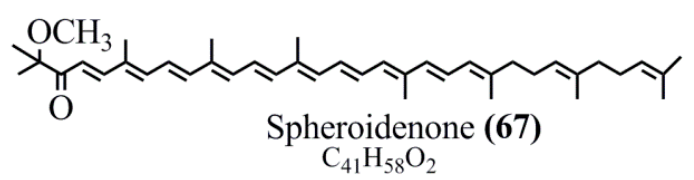

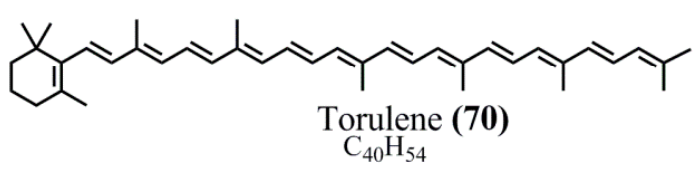

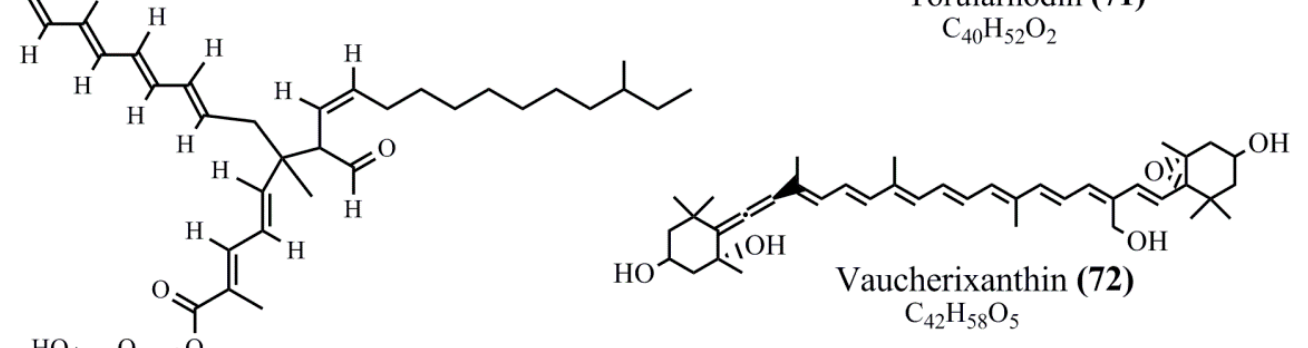<smiles>O[C@H]1O[C@H](O)[C@H](O)[C@@H](O)[C@@H]1O</smiles>

Staphyloxanthin (69)

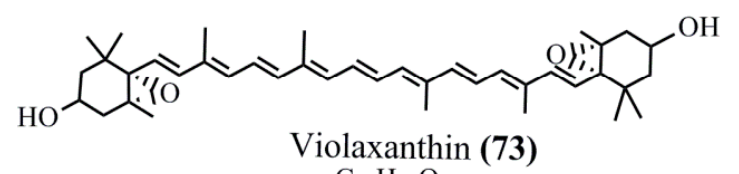
$\mathrm{C}_{51} \mathrm{H}_{78} \mathrm{O}_{8}$ $\mathrm{C}_{40} \mathrm{H}_{56} \mathrm{O}_{4}$<smiles>[Y]c1c(OC)cc2cc3c(c(O)c2c1O)C(=O)O[C@H](C)C3</smiles><smiles>[Y4]C=CC=CC=CC=CC=CC=CC=CC=CC(=O)O</smiles>

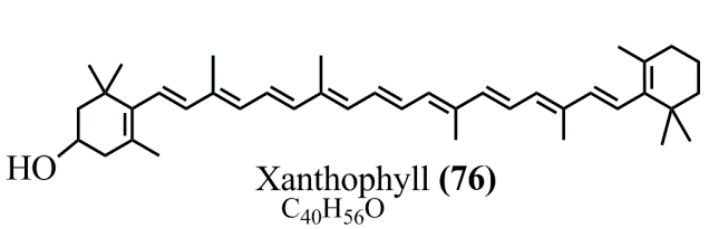

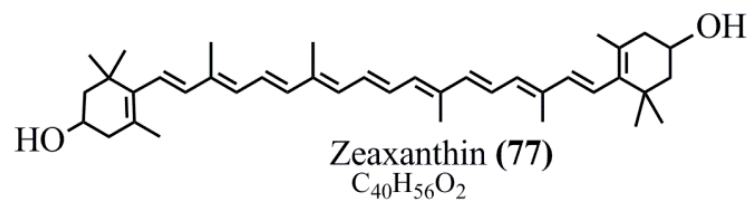

Figure 1. Cont. 
<smiles>N=C(N)NCCCC(NC(=O)CC(N)C(=O)O)C(=O)O</smiles><smiles>C/C=C1\C(=Cc2[nH]c(/C=C3\N=C(/C=C4/NC(=O)C(C)C4CC)C(C)=C3CCC(=O)O)c(CCC(=O)O)c2C)NC(=O)C1CC</smiles>

Phycocyanin (79) $\mathrm{C}_{10} \mathrm{H}_{19} \mathrm{~N}_{5} \mathrm{O}_{5}$<smiles>CCC1=C(C)/C(=C\C2=N/C(=C\c3[nH]c(/C=C4/NC(=O)C(C)/C4=C(/C)SC[C@H](NC(N)=O)C(=O)O)c(C)c3CCC(=O)O)C(CCC(=O)O)=C2C)NC1=O</smiles><smiles>C=CC1=C(C)[C@H](CC2=N/C(=C\c3[nH]c(/C=C4/NC(=O)[C@@H](C)[C@H]4[C@H](C)[C@H](C)S)c(C)c3CCC(=O)O)C(CCC(=O)O)=C2C)NC1=O</smiles><smiles>C=CC1C(=O)NC(CC2=NC(=Cc3[nH]c(C=C4NC(=O)C(C)C4=CC)c(C)c3CCC(=O)O)C(CCC(=O)O)=C2C)C1C</smiles>

Phycoerythrobilin (82)

$$
\mathrm{C}_{33} \mathrm{H}_{40} \mathrm{~N}_{4} \mathrm{O}_{6}
$$

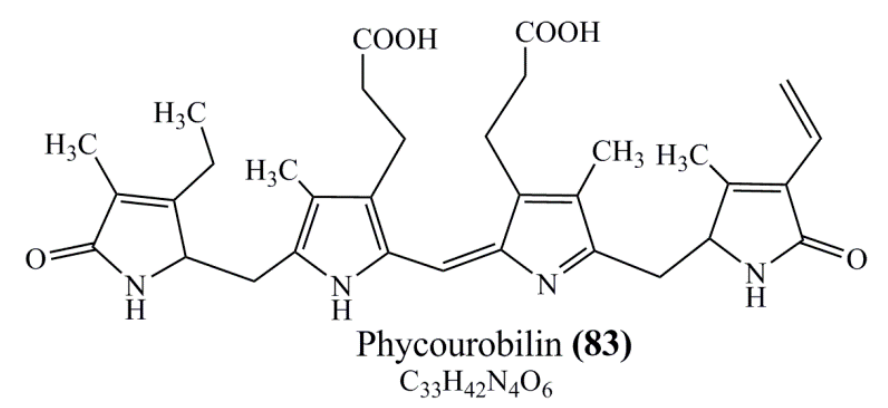

Figure 1. Cont. 


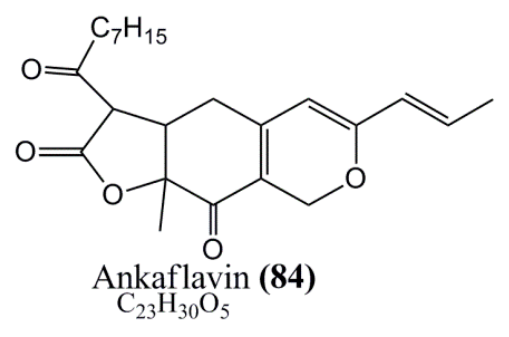<smiles>C/C=C\C1=CC2=C(CO1)C(=O)C1(C)OC(=O)C(C(=O)CCCCC)C1C2</smiles><smiles>Cc1cc2nc3c(=O)[nH]c(=O)nc-3n(CC(O)C(O)C(O)CO)c2cc1C</smiles>

Riboflavin (86)<smiles>Cc1cc2nc3c(=O)[nH]c(=O)nc-3n(CC(O)C(O)C(O)CO)c2cc1N(C)C</smiles>

Roseoflavin (87)

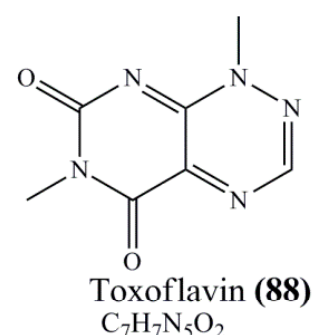
$\mathrm{C}_{17} \mathrm{H}_{20} \mathrm{~N}_{4} \mathrm{O}_{6}$ $\mathrm{C}_{18} \mathrm{H}_{23} \mathrm{~N}_{5} \mathrm{O}_{6}$<smiles>Oc1cccc2cccc(O)c12</smiles>

DHN (90)

$\mathrm{C}_{10} \mathrm{H}_{8} \mathrm{O}_{2}$<smiles>NC(CC1=CC(=O)C(=O)C=C1)C(=O)O</smiles>

DOPA (91)

$\mathrm{C}_{9} \mathrm{H}_{9} \mathrm{NO}_{4}$<smiles></smiles>

Eumelanin (92)<smiles>CCC[C@H]1C[C@@H](C(=O)N[C@H](C(C)O)[C@]2(C)OC(SC)C(O)C(O)C2O)N(C)C1</smiles>

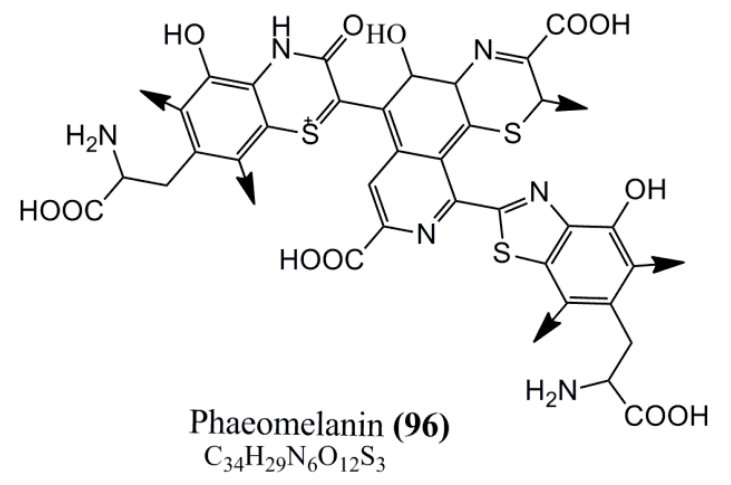<smiles>COC(=O)c1cccc2nc3c(C(C)C(C)c4cccc5nc6cccc(C(=O)OC)c6nc45)cccc3nc12</smiles><smiles>CC1=C(/C=C/C(C)=C/C=C/C(C)=C/CO)C(C)(C)CCC1</smiles>

All trans retinal (98) $\mathrm{C}_{20} \mathrm{H}_{30} \mathrm{O}$

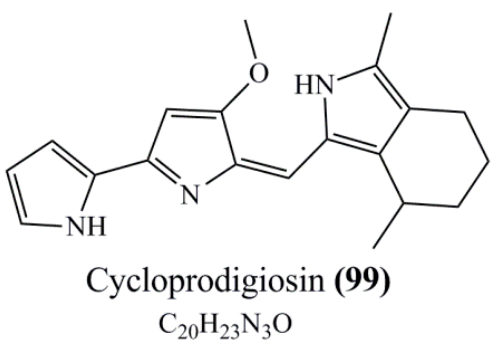

Figure 1. Cont. 


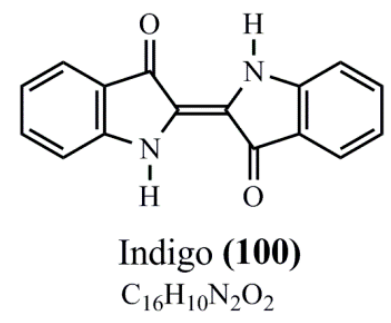<smiles>CCCCCCCCCCCc1ccc(/C=C2\N=C(c3ccc[nH]3)C=C2OC)[nH]1</smiles>

Undecylprodigiosin (103) $\mathrm{C}_{25} \mathrm{H}_{35} \mathrm{~N}_{3} \mathrm{O}$

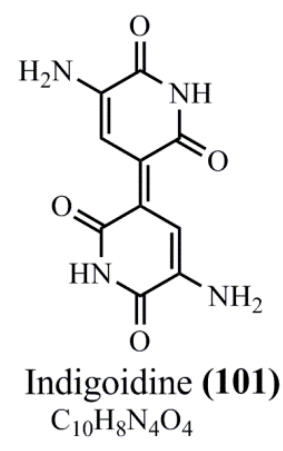

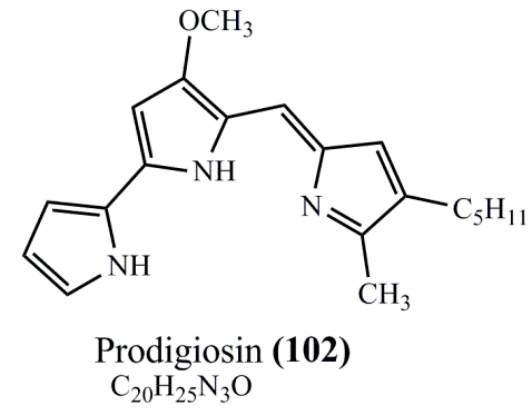<smiles>O=C1NC(c2c[nH]c3ccc(O)cc23)=C/C1=C1/C(=O)Nc2ccccc21</smiles>

Violacein (104) $\mathrm{C}_{20} \mathrm{H}_{13} \mathrm{~N}_{3} \mathrm{O}_{3}$<smiles>NC(=O)c1cccc2c1Nc1ccccc1N2</smiles>

Chlororaphine (106) $\mathrm{C}_{13} \mathrm{H}_{11} \mathrm{~N}_{3} \mathrm{O}$

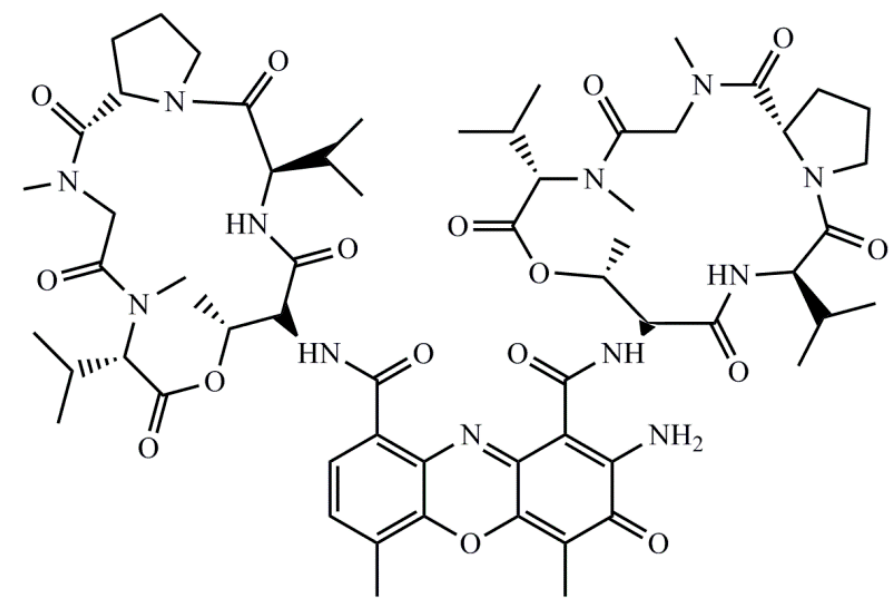

Actinomycin D (105)

$\mathrm{C}_{62} \mathrm{H}_{86} \mathrm{~N}_{12} \mathrm{O}_{16}$<smiles>COC(=O)c1cccc2c1Nc1cccc(C(=O)O)c1N2</smiles>

Dihydrophencomycin (107) $\mathrm{C}_{15} \mathrm{H}_{12} \mathrm{~N}_{2} \mathrm{O}_{4}$<smiles>COc1ccc(COC(O)CO)c2nc3cccc(C(=O)O)c3nc12</smiles>

Griseolutein (108) $\mathrm{C}_{17} \mathrm{H}_{16} \mathrm{~N}_{2} \mathrm{O}_{6}$<smiles>CN1c2cccc(O)c2N([O])C2C(O)=CC=CC21</smiles>

Iodinin (109) $\mathrm{C}_{12} \mathrm{H}_{8} \mathrm{~N}_{2} \mathrm{O}_{4}$

Figure 1. Cont. 
<smiles>COc1cccc2c1[n+]([O-])c1cccc(O)c1[n+]2[O-]</smiles>

Myxin (Cuprimycin) (110) $\mathrm{C}_{13} \mathrm{H}_{10} \mathrm{~N}_{2} \mathrm{O}_{4}$<smiles>COC(=O)c1cccc2nc3c(C(C)C(C)c4cccc5nc6c(C(=O)OC)cccc6nc45)cccc3nc12</smiles>

Phenozostatin D (113)<smiles>NC(=O)c1cccc2nc3ccccc3nc12</smiles>

Oxychlororaphine (111) $\mathrm{C}_{13} \mathrm{H}_{9} \mathrm{~N}_{3} \mathrm{O}$<smiles>NC(=O)c1cccc2nc3ccccc3nc12</smiles>

Phenazine-1-carboxylic acid (112) $\mathrm{C}_{13} \mathrm{H}_{9} \mathrm{~N}_{3} \mathrm{O}$ $\mathrm{C}_{32} \mathrm{H}_{26} \mathrm{~N}_{4} \mathrm{O}_{4}$<smiles>Cn1c2cccc(=O)c-2nc2ccccc21</smiles>

Pyocyanin (114) $\mathrm{C}_{13} \mathrm{H}_{10} \mathrm{~N}_{2} \mathrm{O}$<smiles></smiles>

Pyorubin (Aeruginosin A+B) (115)

$\mathrm{C}_{28} \mathrm{H}_{22} \mathrm{~N}_{6} \mathrm{O}_{7} \mathrm{~S}$

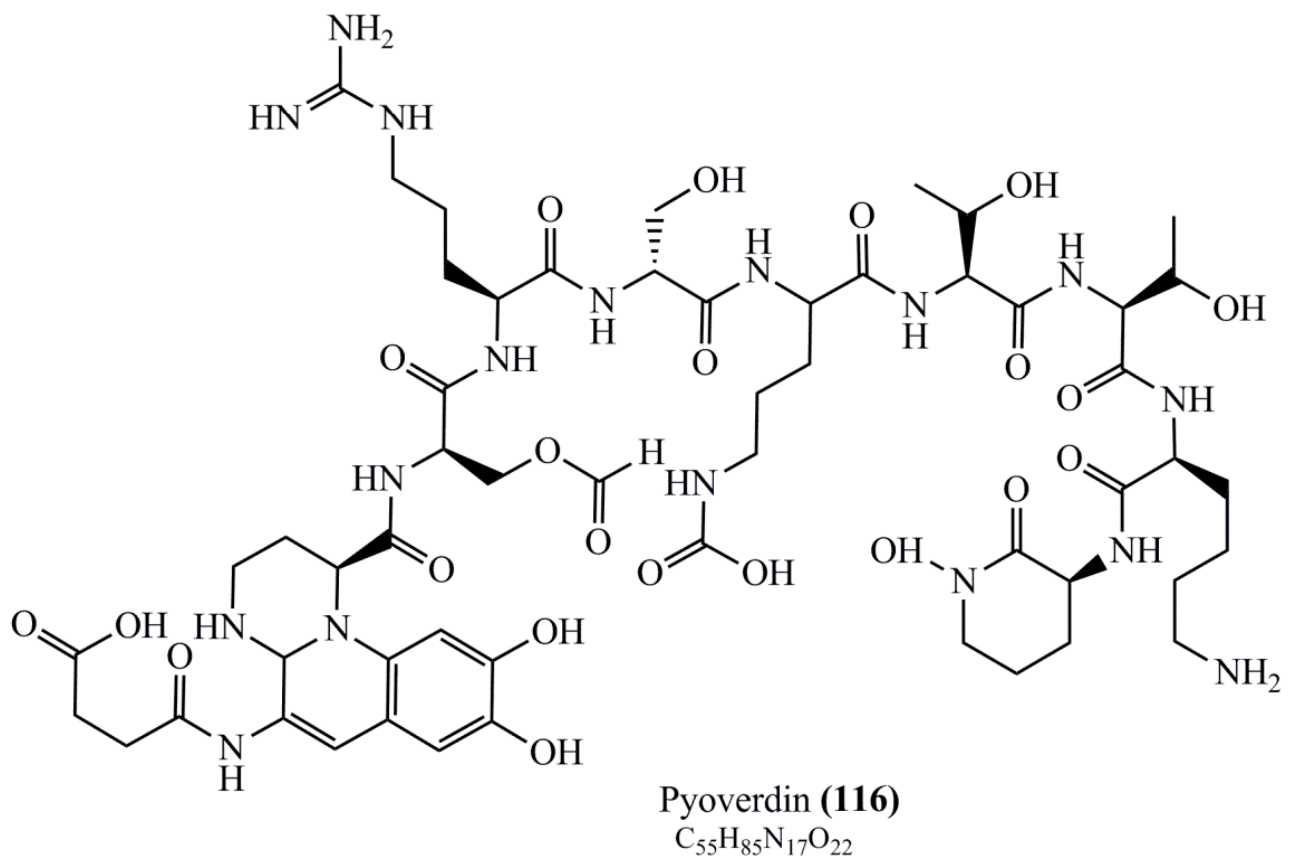

Figure 1. Cont. 
<smiles>C=C(C)/C=C/c1c(O)cc(CC)c2c1C(=O)c1cc(O)c(C(=O)O)cc1C2=O</smiles>

Arpink Red (117) $\mathrm{C}_{22} \mathrm{H}_{18} \mathrm{O}_{6}$<smiles>COc1cc(O)c2c(c1O)C(=O)c1cc(C)ccc1C2=O</smiles>

Austrocortinin (119)

$$
\mathrm{C}_{16} \mathrm{H}_{12} \mathrm{O}_{5}
$$<smiles>Cc1cc(O)c2c(c1O)C(=O)c1c(O)cc(O)cc1C2=O</smiles><smiles>CC1=C(C(=O)/C=C/CC(C)(C)C/C=C/C(C)C)C(=O)c2ccccc2C1=O</smiles><smiles>Cc1cc(O)c2c(c1)C(=O)c1cccc(O)c1C2=O</smiles>

Chrysophanol (125)

$$
\mathrm{C}_{15} \mathrm{H}_{10} \mathrm{O}_{4}
$$<smiles>CCC/C=C\Cc1c(O)cc2c(c1O)C(=O)c1c(O)cc(O)cc1C2=O</smiles>

Averythrin (118)

$\mathrm{C}_{20} \mathrm{H}_{18} \mathrm{O}_{6}$<smiles>COc1cc(O)c2c(c1O)C(=O)c1cc(C)ncc1C2=O</smiles>

Bostrycoidin (120)

$\mathrm{C}_{15} \mathrm{H}_{11} \mathrm{NO}_{5}$<smiles></smiles>

Cercosporin (122)

$$
\mathrm{C}_{29} \mathrm{H}_{26} \mathrm{O}_{10}
$$<smiles>Cc1cc2c(c(O)c1Cl)C(=O)c1c(O)cc(O)cc1C2=O</smiles><smiles>O=C1c2cc(CO)cc(O)c2C(=O)c2cc(O)cc(O)c21</smiles>

Figure 1. Cont. 
<smiles>Cc1cc(O)c2c(c1O)C(=O)c1c(O)ccc(O)c1C2=O</smiles>

Cynodontin (127) $\mathrm{C}_{15} \mathrm{H}_{10} \mathrm{O}_{6}$<smiles>COc1c(O)c(O)c2c(c1O)C(=O)c1cc(C)cc(O)c1C2=O</smiles>

Dermocybin (128) $\mathrm{C}_{16} \mathrm{H}_{12} \mathrm{O}_{7}$<smiles>Cc1cc(O)c2c(c1)C(=O)c1cc(C)c(O)c(O)c1C2=O</smiles>

Dermoglaucin (129) $\mathrm{C}_{16} \mathrm{H}_{12} \mathrm{O}_{6}$<smiles>COc1cc(O)cc2c1C(=O)c1c(O)c(C(=O)O)c(C)c(O)c1C2=O</smiles>

Dermorubin (130)<smiles>COC1=CC(=O)c2c(O)cc(OC)c(O)c2C1=O</smiles>

Draconin (131)

$\mathrm{C}_{12} \mathrm{H}_{10} \mathrm{O}_{6}$<smiles>Cc1cc(O)c2c(c1O)C(=O)c1cc(CO)cc(O)c1C2=O</smiles>

Erythroglaucin (134) $\mathrm{C}_{16} \mathrm{H}_{12} \mathrm{O}_{6}$<smiles>C[C@@]1(O)CC(=O)c2c(cc3cc(O)c(-c4c(O)cc5cc6c(c(O)c5c4O)C(=O)C[C@](C)(O)C6)c(O)c3c2O)C1</smiles>

$\underset{\mathrm{C}_{30} \mathrm{H}_{26} \mathrm{O}_{10}}{\text { Flavomannin }}$ (137)<smiles></smiles>

Elsinochrome A (132) $\mathrm{C}_{30} \mathrm{H}_{24} \mathrm{O}_{10}$
Fallacinal (135)

$\mathrm{C}_{16} \mathrm{H}_{10} \mathrm{O}_{6}$<smiles>Cc1cc(O)c2c(c1)C(=O)c1cc(O)cc(O)c1C2=O</smiles>

Emodin (133)

$\mathrm{C}_{15} \mathrm{H}_{10} \mathrm{O}_{5}$<smiles>O=C1C=C(O)c2c(O)cc(O)cc2C1=O</smiles>

$\underset{\mathrm{C}_{10} \mathrm{H}_{6} \mathrm{O}_{5}}{\text { Flaviolin (136) }}$<smiles>Cc1cc(O)c2c(c1)C(=O)c1c(O)ccc(O)c1C2=O</smiles>

Helminthosporin (139) $\mathrm{C}_{15} \mathrm{H}_{10} \mathrm{O}_{5}$<smiles>COC1=CC(=O)c2c(O)c(C)c(CC(C)=O)c(O)c2C1=O</smiles>

Javanicin (140)

$\mathrm{C}_{15} \mathrm{H}_{14} \mathrm{O}_{6}$<smiles>O=C1C=CC(=O)c2c(O)cccc21</smiles>

Juglone (141) $\mathrm{C}_{10} \mathrm{H}_{6} \mathrm{O}_{3}$<smiles>CC1=CC(=O)c2c(O)c3c(c(O)c2C1=O)CC(C)(O)OC3</smiles>

Fusarubin (138) $\mathrm{C}_{15} \mathrm{H}_{14} \mathrm{O}_{7}$<smiles>CC(=O)Cc1c(C)c(O)c2c(c1O)C(=O)CCC2=O</smiles>

Karuquinone A (142) $\mathrm{C}_{14} \mathrm{H}_{14} \mathrm{O}_{5}$<smiles>C=C(C)CCCC1=C(C)C(=O)c2ccccc2C1=O</smiles>

Menaquinone-7 (143) $\mathrm{C}_{46} \mathrm{H}_{64} \mathrm{O}_{2}$

Figure 1. Cont. 
<smiles>O=C1C=CC(=O)c2ccccc21</smiles>

Naphthoquinone (144) $\mathrm{C}_{10} \mathrm{H}_{6} \mathrm{O}_{2}$<smiles>COc1cc(O)c2c(c1)C(=O)c1cc(C(=O)O)cc(O)c1C2=O</smiles>

Parietinic acid (147)

$\mathrm{C}_{16} \mathrm{H}_{10} \mathrm{O}_{7}$<smiles>Cc1cc(O)c2c(c1)C(=O)c1cc(CO)cc(O)c1C2=O</smiles>

Physcion (150)

$\mathrm{C}_{16} \mathrm{H}_{12} \mathrm{O}_{5}$<smiles>C/C(O)=C/c1cc2c(c(O)c1=CO)C(=O)C=C(CO)C=2</smiles>

Nectriachrysone (145) $\mathrm{C}_{15} \mathrm{H}_{14} \mathrm{O}_{5}$<smiles>CCC(C)C(=O)C1=C(NCCO)C(C)(O)C(=O)C(C)=C1OC</smiles>

Phomaligin A (148) $\mathrm{C}_{16} \mathrm{H}_{25} \mathrm{NO}_{5}$<smiles>Cc1ccc2c(c1O)C(=O)c1ccccc1C2=O</smiles>

Pachybasin (146) $\mathrm{C}_{15} \mathrm{H}_{10} \mathrm{O}_{3}$<smiles>Cc1cc(O)c2c(c1)C(=O)c1cc(O)ccc1C2=O</smiles>

Phomarin (149)

$\mathrm{C}_{15} \mathrm{H}_{10} \mathrm{O}_{4}$<smiles>Cc1c(C(=O)CCC(C)C)cc2c(c1O)C(=O)C(=O)c1c(O)cccc1-2</smiles>

Piloquinone (151)<smiles>COc1cc(O)cc2c1C(=O)c1cc(C)cc(O)c1C2=O</smiles>

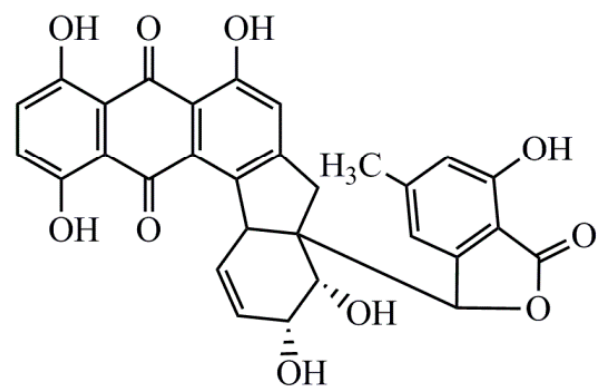<smiles>COc1cc(O)cc2c1C(=O)c1c(O)cc(C)c(O)c1C2=O</smiles>

$\underset{\mathrm{C}_{16} \mathrm{H}_{12} \mathrm{O}_{6}}{\text { Rubrocrin }_{6}}(\mathbf{1 5 4})$

Rubellin D (153)

$\mathrm{C}_{30} \mathrm{H}_{22} \mathrm{O}_{10}$<smiles></smiles><smiles>COC1=C(O)C(=O)C(O)=C(C)C1=O</smiles>

Spinulosin (156) $\mathrm{C}_{8} \mathrm{H}_{8} \mathrm{O}_{5}$

Figure 1. Cont. 
<smiles>O=C1c2cc(CO)cc(O)c2C(=O)c2c(O)cc(CO)cc21</smiles>

Teloschistin (157)

$\mathrm{C}_{16} \mathrm{H}_{12} \mathrm{O}_{6}$<smiles>O=C1c2cc(O)cc(O)c2C(=O)c2c(O)c(CO)cc(O)c21</smiles>

Tritisporin (158)

$\mathrm{C}_{15} \mathrm{H}_{10} \mathrm{O}_{7}$

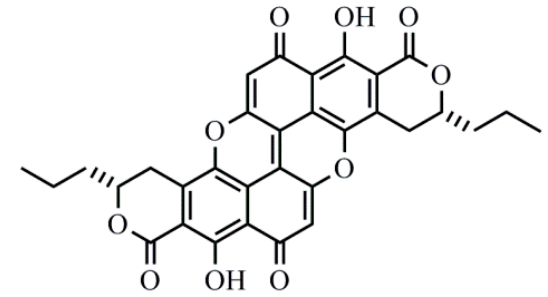

Xylindein (159)

$\mathrm{C}_{32} \mathrm{H}_{24} \mathrm{O}_{10}$<smiles>CCC(C)C(=O)OC1CC(C)C=C2C=CC(C)C(CCC3CC(O)CC(=O)O3)C21</smiles>

Lovastatin (160) $\mathrm{C}_{24} \mathrm{H}_{36} \mathrm{O}_{5}$<smiles>C/C=C/C1=CC2=CC3=C(C(=O)CCCCC)C(=O)OC3(C)C(=O)C2=CO1</smiles>

Monascorubrin (163) $\mathrm{C}_{23} \mathrm{H}_{26} \mathrm{O}_{5}$<smiles>CC(=O)c1cccc2cccc-2c1</smiles>

Acetylazulene (166) $\mathrm{C}_{12} \mathrm{H}_{10} \mathrm{O}$<smiles>CC1OC(N2/C(=C3\Nc4ccc(Cl)cc4C3=O)C(=O)c3cc(Cl)ccc32)C(O)C(O)C1N</smiles>

Akashin (168)

$\mathrm{C}_{22} \mathrm{H}_{19} \mathrm{Cl}_{2} \mathrm{~N}_{3} \mathrm{O}_{5}$

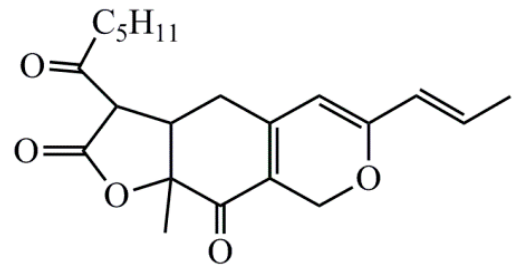

Monascin (161)

$\mathrm{C}_{21} \mathrm{H}_{26} \mathrm{O}_{5}$<smiles>C/C=C/C1=CC2=CC3=C(C(=O)CCCC)C(=O)OC3(C)C(=O)C2=CN1</smiles>

Rubropunctamine (164) $\mathrm{C}_{21} \mathrm{H}_{23} \mathrm{NO}_{4}$<smiles>C/C=C/C1=CC2=CC3=C(C(=O)CCC)C(=O)OC3(C)C(=O)C2=CN1C(CCC(=O)O)C(=O)O</smiles>

Monascorubramine (162) $\mathrm{C}_{28} \mathrm{H}_{33} \mathrm{NO}_{8}$

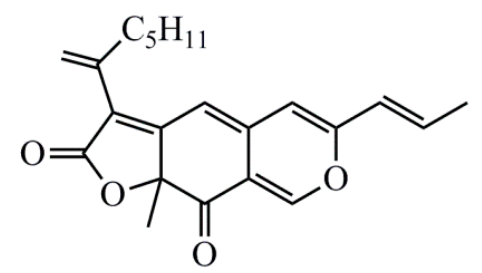

Rubropunctatine (165) $\mathrm{C}_{22} \mathrm{H}_{24} \mathrm{O}_{4}$<smiles>CC1OC(CC(=O)O)CC2=C1C(=O)c1c(O)c(-c3cc(O)c4c(c3O)C(=O)C3=C(CC(CC(=O)O)OC3C)C4=O)cc(O)c1C2=O</smiles>

Actinorhodin (167)

$\mathrm{C}_{32} \mathrm{H}_{26} \mathrm{O}_{14}$<smiles>Cc1occ2c1C(=O)C(=O)C(C(C)(C)C)=C2</smiles>

$\mathrm{HO}^{\prime}$<smiles>O=C1C[C@@H](O)[C@H]2c3c(ccc(O)c31)-c1ccc(O)c3c1[C@@]2(O)C=CC3=O</smiles>

Alterperylenol (170)

$\mathrm{C}_{20} \mathrm{H}_{14} \mathrm{O}_{6}$

Figure 1. Cont. 
<smiles>CC(C)=CCC/C(C)=C/CC/C(C)=C/CC/C(C)=C/CC1=C(O)C(=O)C(CC2=C(O)C(=O)C(C/C=C(\C)CC/C=C(\C)CC/C=C(\C)CCC=C(C)C)=C(O)C2=O)=C(O)C1=O</smiles>

$\underset{\mathrm{C}_{53} \mathrm{H}_{72} \mathrm{O}_{8}}{\text { Amitenone (171) }}$<smiles>CN1C(=S)c2cc(C(N)=O)nc3c(N)c(Cl)c(N)c1c23</smiles>

Ammosamide A (172)

$\mathrm{C}_{12} \mathrm{H}_{10} \mathrm{ClN}_{5} \mathrm{OS}$<smiles>CN1C(=O)c2cc(C(N)=O)nc3c(N)c(Cl)c(N)c1c23</smiles>

Ammosamide B (173)

$\mathrm{C}_{12} \mathrm{H}_{10} \mathrm{ClN}_{5} \mathrm{O}_{2}$<smiles>COC(=O)c1c(C)cc(OC(=O)c2c(C)cc(O)c(C=O)c2O)c(C)c1O</smiles><smiles>O=C1C(O)=C(c2ccc(O)cc2)C(=O)C(O)=C1c1ccc(O)cc1</smiles>

Atranorin (174)

$\mathrm{C}_{19} \mathrm{H}_{18} \mathrm{O}_{8}$<smiles>COc1nsc2c1C(=O)c1c(O)cccc1C2=O</smiles>

Aulosirazole (176) $\mathrm{C}_{12} \mathrm{H}_{7} \mathrm{NO}_{4} \mathrm{~S}$<smiles>C/C=C1/C=C2C=C(C)C(=O)[C@](C)(O)[C@]2(OC(=O)c2c(C)cc(O)cc2O)CO1</smiles>

Azaphilone (179)

$\mathrm{C}_{21} \mathrm{H}_{22} \mathrm{O}_{7}$<smiles>O=c1oc2cc3cc(O)c(O)cc3oc-2c1-c1ccc(O)cc1</smiles>

$\mathrm{C}_{17} \mathrm{H}_{10} \mathrm{O}_{6}$

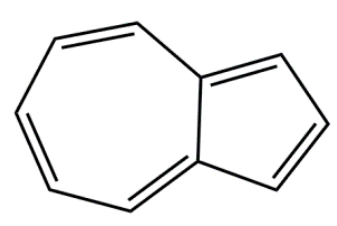

Azulene (180)

$\mathrm{C}_{10} \mathrm{H}_{8}$<smiles>COCc1cc(C)c2c(O)c3c(=O)cc(C)oc3c(-c3c(C)cc4cc5oc(C)cc(=O)c5c(O)c4c3OC)c2c1</smiles>

Aurasperone A (178) $\mathrm{C}_{32} \mathrm{H}_{26} \mathrm{O}_{10}$<smiles>CCCC1=C(O)C(=O)C=C(O)C1=O</smiles>

Boviquinone 3 (181) $\mathrm{C}_{21} \mathrm{H}_{28} \mathrm{O}_{4}$

Figure 1. Cont. 
<smiles>[R]C(NC(C)(C)C)C(=O)OC(C)C(NC(=O)C(NC(=O)/C=C/C=C/C=C/C=C/c1ccc(O)c(Br)c1)C(C)C(C)C)C(=O)NC(CC(N)=O)C(=O)NC(CC(N)=O)C(=O)NC([R])C(C)(C)C</smiles>

Bromo-alterochromide (182)

$\mathrm{C}_{35} \mathrm{H}_{44} \mathrm{BrN}_{7} \mathrm{O}_{10} \mathrm{R}$

Calycin (183)

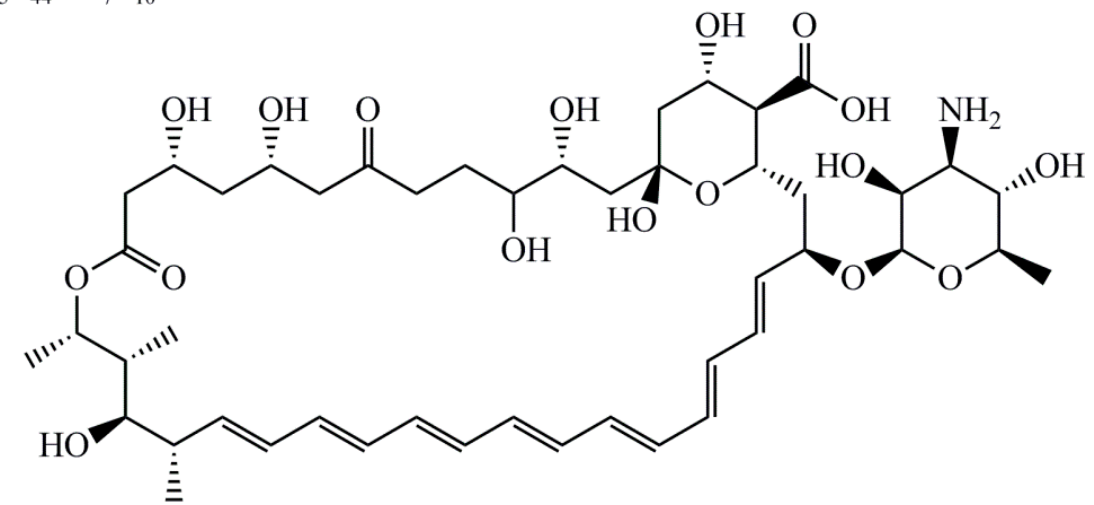

Candidin (184)

$\mathrm{C}_{47} \mathrm{H}_{71} \mathrm{NO}_{17}$<smiles>COC(=O)/C=C/C=C/C=C/C=C/C=C/C=C/C=C/c1ccc(Cl)c(O)c1C</smiles>

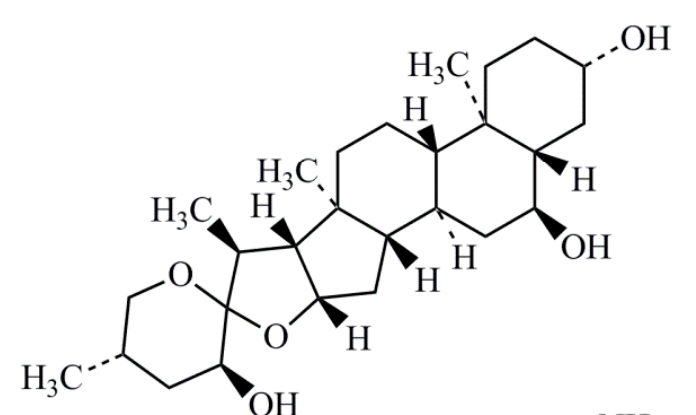<smiles>CC1=C2C(=COC(C)[C@@H]2C)C(O)=C(C(=O)O)C1=O</smiles>

$\underset{\mathrm{C}_{13} \mathrm{H}_{14} \mathrm{O}_{5}}{\mathrm{Citrinin}}(\mathbf{1 8 7})$

Chrysogenin (186)<smiles>CC(C)=CCc1ccc2[nH]cc(C3=C(O)C(=O)C(c4c[nH]c5ccc(CC=C(C)C)cc45)=C(O)C3=O)c2c1</smiles><smiles>Nc1ncnc2c1ncn2C1COC(CO)C1O</smiles><smiles>CCCC(C)(C)OC(=O)C(C)(C)C(=O)O</smiles>

Figure 1. Cont. 
<smiles></smiles>

Diastaphenazine (191) $\mathrm{C}_{26} \mathrm{H}_{16} \mathrm{~N}_{4} \mathrm{O}_{4}$

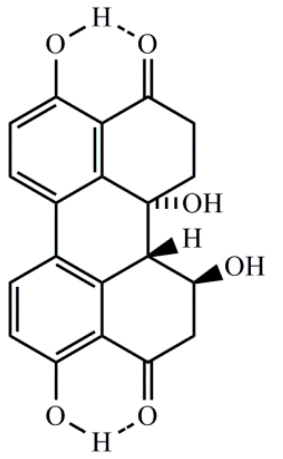

Dihydroalterperylenol (192)<smiles></smiles>

Dihydroxyazulene (193) $\mathrm{C}_{15} \mathrm{H}_{16} \mathrm{O}_{3}$<smiles>CCC(C)C(C(CC(=O)N1CCCC1C(OC)C(C)C(=O)NN(C(=O)O)c1nccs1)OC)N(C)C(=O)C(NC(=O)C(C(C)C)N(C)C)C(C)C</smiles><smiles>C/C=C/C=C/C=C/C(=O)CC(O)C1=C2C=C3C[C@H](CO)OC=C3C(=O)[C@@]2(C)OC1=O</smiles><smiles>COc1c2c(cc3cc(O)cc(C)c13)OC(C)(O)CC2=O</smiles>

Fonsecin (198)

$\mathrm{C}_{15} \mathrm{H}_{14} \mathrm{O}_{6}$<smiles></smiles>

Glaukothalin (201) $\mathrm{C}_{35} \mathrm{H}_{59} \mathrm{~N}_{4} \mathrm{O}_{4}$

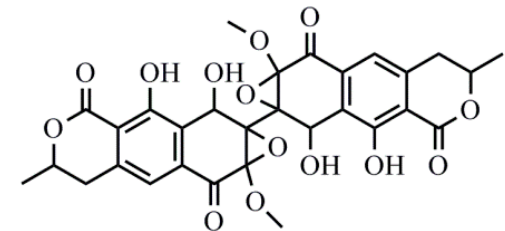

Floccosin (196) $\mathrm{C}_{30} \mathrm{H}_{26} \mathrm{O}_{14}$<smiles>O=C(O)c1ccccc1-c1c2ccc(=O)cc-2oc2cc(O)ccc12</smiles>

Fluorescein (197) $\mathrm{C}_{20} \mathrm{H}_{12} \mathrm{O}_{5}$

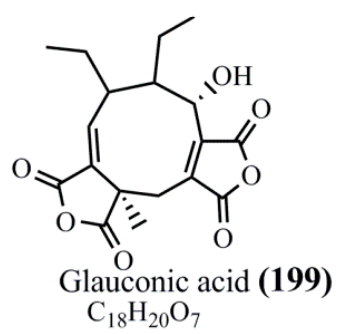

$\mathrm{C}_{18} \mathrm{H}_{20} \mathrm{O}_{7}$<smiles>O=C1O/C(=C(\C(=O)O)c2ccc(O)c(O)c2)C(O)=C1c1cc(O)c(O)c(O)c1</smiles>

Gomphidic acid (200) $\mathrm{C}_{18} \mathrm{H}_{12} \mathrm{O}_{9}$<smiles>C/C(=C\C=C\C(=O)NC(CCCN)C(=O)O)OC1OC(C)C(O)C(O)C1O</smiles>

Figure 1. Cont. 
<smiles>CC(C)(C)[C@H]1CCCCC1=O</smiles><smiles>O=C1C=C2c3c(c[nH]c3C1)C[C@H]2O</smiles>
$\underset{\mathrm{C}_{13} \mathrm{H}_{14} \mathrm{~N}_{2} \mathrm{O}_{2}}{\text { Haematopodin (205) }}$<smiles></smiles>

Hypericin (207) $\mathrm{C}_{30} \mathrm{H}_{16} \mathrm{O}_{8}$<smiles>C=C(C)c1ccc(C)c2ccc(C=O)c-2c1</smiles>

$\underset{\mathrm{C}_{15} \mathrm{H}_{14} \mathrm{O}}{\operatorname{Lachin}}$ (209)<smiles>COC1=CC2(OC(=O)c3c(NC4=CC(=O)C(N)=C(C(=O)O)C4=O)ccc(O)c32)C(=O)C(N)=C1N</smiles>

Lilacinone (211)

$\mathrm{C}_{22} \mathrm{H}_{15} \mathrm{~N}_{3} \mathrm{O}_{11}$<smiles>Cc1cc(OC(=O)c2c(C)cc(OC(=O)c3c(C)cc(O)cc3O)cc2O)cc(O)c1C(=O)O</smiles>

$\underset{\mathrm{C}_{24} \mathrm{H}_{20} \mathrm{O}_{10}}{\mathrm{G}}$<smiles>O=C1CCC(O)=C1NC(=O)c1cc2cc3c(c(O)c2c(=O)o1)O[C@]1(CC3)Cc2c(O)c3c(c(O)c2O1)C(=O)C(CO)=CC3=O</smiles><smiles>C[C@H]1Cc2c(O)c3c(c(O)c2C(=O)O1)C(=O)C(O)=C(C1=C(O)C(=O)c2c(O)c4c(c(O)c2C1=O)C(=O)O[C@H](C)C4)C3=O</smiles>

Iridosporin (208) $\mathrm{C}_{28} \mathrm{H}_{18} \mathrm{O}_{14}$<smiles>CC(=O)/C(C)=C/C=C\C=C/C=C/C=C/C=C/C=C/C=C/C=C/CC(O)CC(=O)O</smiles>
$\underset{\mathrm{C}_{27} \mathrm{H}_{32} \mathrm{O}_{4}}{\mathrm{~A}}$<smiles>C[C@H]1Cc2cc3c(c(O)c2C(=O)O1)C(=O)C(C1=C(O)C(=O)c2cc4c(c(O)c2C1=O)C(=O)O[C@H](C)C4)=C(O)C3=O</smiles>

Luteosporin (212) $\mathrm{C}_{28} \mathrm{H}_{18} \mathrm{O}_{12}$

Figure 1. Cont. 
<smiles>[M]OC1=C(C(=O)CCCCC)C(=O)N(C(C)=O)/C1=C/C</smiles>

Magnesidin (213) $\mathrm{C}_{28} \mathrm{H}_{36} \mathrm{MgN}_{2} \mathrm{O}_{8}$

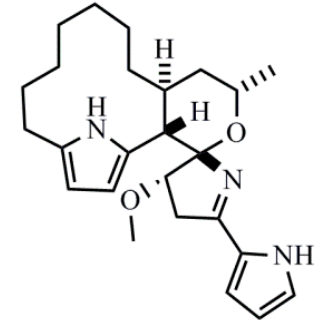
$\underset{\mathrm{C}_{25} \mathrm{H}_{35} \mathrm{~N}_{3} \mathrm{O}_{2}}{\operatorname{Marineosin}} \mathrm{A}(\mathbf{2 1 4})$<smiles>CC(C)=CCCCC1OC2=C(C(=O)c3c(O)cc(O)c(Br)c3C2=O)C2CC=C(C)CCC12</smiles>

Marinone (215)

$\mathrm{C}_{25} \mathrm{H}_{27} \mathrm{BrO}_{5}$<smiles>CC(=O)/C(C)=C/C=C/C=C/C=C/C=C/C=C/C=C/CC(=O)N[C@@H](Cc1ccccc1)C(C)=O</smiles>

Melanocrocin (216) $\mathrm{C}_{29} \mathrm{H}_{33} \mathrm{NO}_{4}$<smiles>CC(=O)N[C@@H](CCO[C@H](C)C/C=C/CC[C@@H](O)CC(C)=Cc1cc(O)c(CC[C@H](N)C(=O)O)c(=O)o1)C(C)=O</smiles>

Mycenaaurin A (218)

$\mathrm{C}_{36} \mathrm{H}_{48} \mathrm{~N}_{2} \mathrm{O}_{10}$<smiles>COC(=O)/C=C/C=C/C=C/C=C/C=C/C=C/C=C/c1cccc(O)c1C</smiles>

Natronochrome (220)

$\mathrm{C}_{23} \mathrm{H}_{24} \mathrm{O}_{3}$<smiles>CN1CCCC1c1cccnc1</smiles>

Nicotine (221)<smiles>C/C=C/C1=CC2=CC(=O)C(C)(OC(=O)c3c(C)cc(OCC)cc3O)C(=O)C2=CO1</smiles>

Mitorubrin (217)

$$
\mathrm{C}_{21} \mathrm{H}_{18} \mathrm{O}_{7}
$$<smiles>CO[C@H]1[C@H](N(C)C(N)=O)C[C@H]2O[C@]1(Cl)n1c3ccccc3c3c4c(c5c6ccccc6n2c5c31)CNC4=O</smiles>

$\mathrm{N}$-carboxamidostaurosporine (219) $\mathrm{C}_{29} \mathrm{H}_{27} \mathrm{~N}_{5} \mathrm{O}_{4}$<smiles></smiles>

Nostocine A (222) $\mathrm{C}_{5} \mathrm{H}_{5} \mathrm{~N}_{5} \mathrm{O}$<smiles>COc1c(Cl)c(C)c(Cl)c(O)c1C(=O)c1c(C=O)c(O)cc(O)c1CC=C(C)C</smiles>

Pestalone (225)

$\mathrm{C}_{21} \mathrm{H}_{20} \mathrm{Cl}_{2} \mathrm{O}_{6}$<smiles>CC(C)CCCCCCCCCCCCc1cc(OS(=O)(=O)O)cc(OS(=O)(=O)O)c1</smiles>

Panosialin (223)<smiles>COc1cc2cc3c(c(O)c2c(O)c1-c1c2c(c(O)c4c(OC)cc(O)cc14)C(=O)CC(C)(O)C2)C(=O)CC(C)(O)C3</smiles>

Phlegmacin A (226) $\mathrm{C}_{32} \mathrm{H}_{30} \mathrm{O}_{10}$

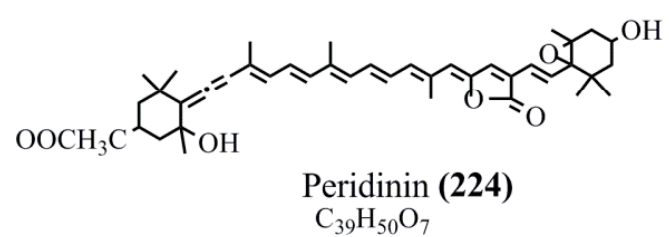

Peridinin (224)<smiles>[R20]Cc1ccccc1OC(=O)ON1c2ccccc2Nc2ccccc21</smiles>

$\underset{\mathrm{C}_{12} \mathrm{H}_{9} \mathrm{NO}}{\text { Phenoxazine (227) }}$

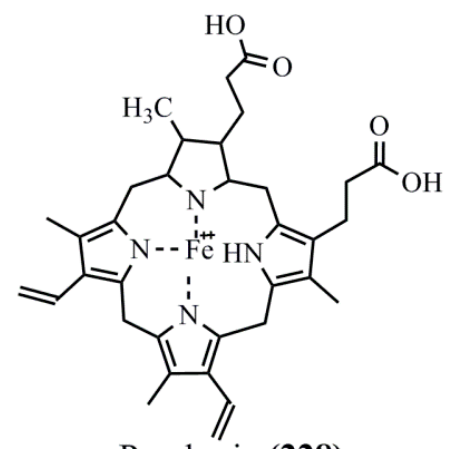

Porphyrin (228)

$\mathrm{C}_{37} \mathrm{H}_{50} \mathrm{FeN}_{4} \mathrm{O}_{4}$

Figure 1. Cont. 
<smiles>O=C1O/C(=C(/C(=O)O)c2ccccc2)C(O)=C1c1ccccc1</smiles>

Pulvinic acid (229)

$\mathrm{C}_{18} \mathrm{H}_{12} \mathrm{O}_{5}$<smiles>CC(=O)N[C@H](C(=O)O[C@H]1CCC(C)(C)[C@H]2CCC3=C(COC3=O)C12C)C(C)C</smiles>

Purpuride (230)

$\mathrm{C}_{22} \mathrm{H}_{33} \mathrm{NO}_{5}$

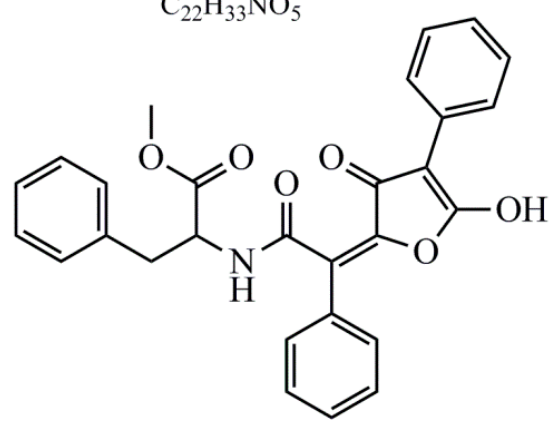

Rhizocarpic acid (233) $\mathrm{C}_{28} \mathrm{H}_{23} \mathrm{NO}_{6}$<smiles>CC1=CC(=O)C(C(=O)Cc2ccccc2)C(=O)O1</smiles>

$\underset{\mathrm{C}_{45} \mathrm{H}_{49} \mathrm{NO}_{8}}{\text { Pyrandione (231) }}$

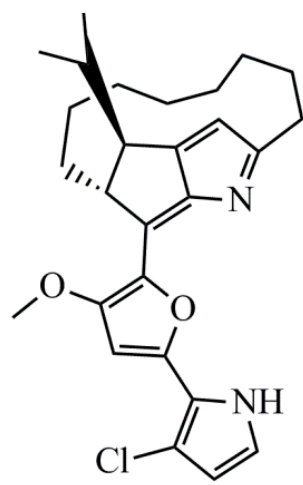

Roseophilin (234) $\mathrm{C}_{27} \mathrm{H}_{33} \mathrm{ClN}_{2} \mathrm{O}_{2}$<smiles>CCCc1nc(C)cc2c1C(=O)c1cc(=O)c3c(c(O)c1-2)O[C@H]1OC(C)C(O)C(O)C31O</smiles>

Rubrolone (235)

$\underset{\mathrm{C}_{31} \mathrm{H}_{37} \mathrm{NO}_{4}}{\text { Pyrrocidine A (232) }}$

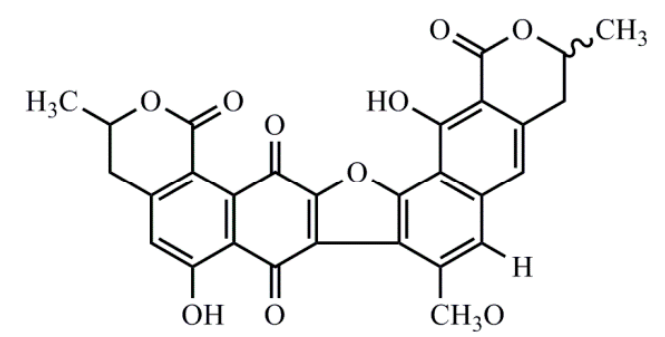

Rubrosulphin (237) $\mathrm{C}_{29} \mathrm{H}_{20} \mathrm{O}_{10}$

Rubrosporin (236)

$\mathrm{C}_{28} \mathrm{H}_{18} \mathrm{O}_{13}$

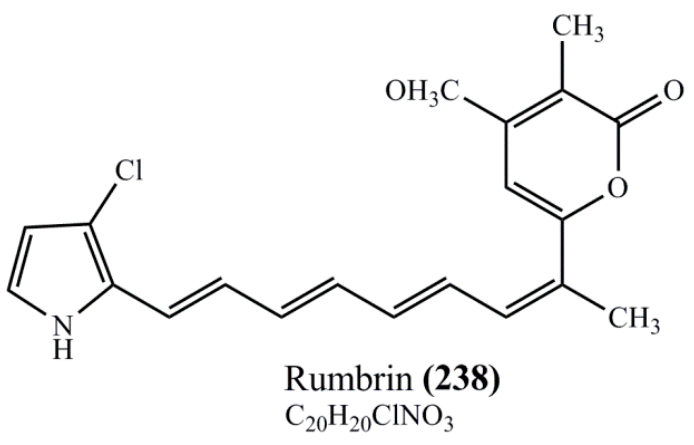

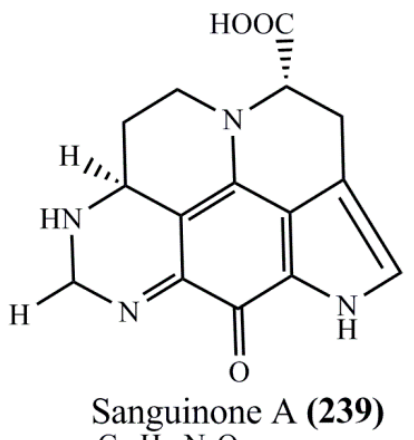

$\mathrm{C}_{15} \mathrm{H}_{14} \mathrm{~N}_{4} \mathrm{O}_{3}$

Figure 1. Cont. 

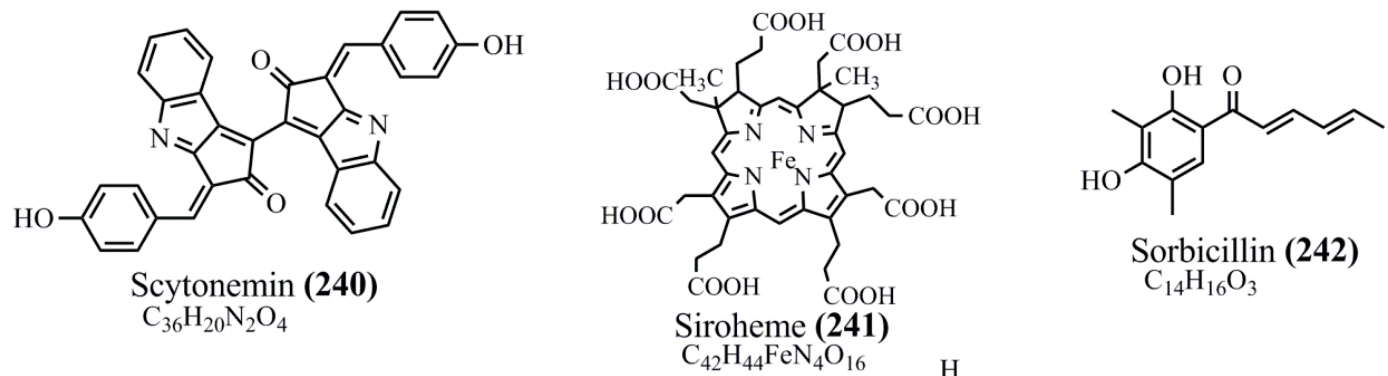<smiles></smiles>

Stearoyldeterrol (243) $\mathrm{C}_{17} \mathrm{H}_{18} \mathrm{O}_{2}$<smiles>CCCCCCCC/C=C\CC/N=C1\N=C(c2ccc[nH]2)C=C1OC</smiles>

Tambjamine (246) $\mathrm{C}_{22} \mathrm{H}_{33} \mathrm{~N}_{3} \mathrm{O}$<smiles>O=C1C=CC(c2c(O)oc3c(-c4ccc(O)c(O)c4)c(O)oc23)=CC1=O</smiles>

Variegatorubin (250) $\mathrm{C}_{18} \mathrm{H}_{10} \mathrm{O}_{8}$<smiles></smiles>

Sterigmatocystin (244) $\mathrm{C}_{18} \mathrm{H}_{12} \mathrm{O}_{6}$<smiles>O=[N+]([O-])O</smiles>

Streptochlorin (245) $\mathrm{C}_{11} \mathrm{H}_{7} \mathrm{ClN}_{2} \mathrm{O}$<smiles>Brc1[nH]c(Br)c(Br)c1Br</smiles>

Tetrabromopyrrole (247) $\mathrm{C}_{4} \mathrm{HBr}_{4} \mathrm{~N}$<smiles>COC(=O)c1cc2cc3cc4cc(CC(=O)O)c(C(=O)C=C(O)c5ccccc5O)c(OC)c4c(OC)c3c(O)c2c(=O)o1</smiles>

$\underset{\mathrm{C}_{32} \mathrm{H}_{24} \mathrm{O}_{12}}{\text { Thermorubin (248) }}$<smiles>O=C1c2ccccc2-n2c1nc1ccccc1c2=O</smiles>

Tryptanthrin (249)<smiles>COC1=C(c2cc3cc4c(c(O)c3c(O)c2OC)C(=O)OC(C)C4)C(=O)c2cc3c(c(O)c21)C(=O)OC(C)C3</smiles>

$\underset{\mathrm{C}_{30} \mathrm{H}_{24} \mathrm{O}_{11}}{\text { Viomellein (251) }}$

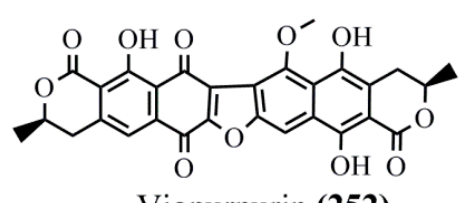

Viopurpurin (252)<smiles>CC(=O)C(=O)C1=C(O)C(c2ccccc2)C(=O)O1</smiles>

Vulpinic acid (253) $\mathrm{C}_{19} \mathrm{H}_{14} \mathrm{O}_{5}$<smiles>COC1=C(C2=C(OC)C(=O)c3cc4c(c(O)c3C2=O)C(=O)OC(C)C4)C(=O)c2cc3c(c(O)c2C1=O)C(=O)OC(C)C3</smiles>

Xanthomegnin (254) $\mathrm{C}_{30} \mathrm{H}_{22} \mathrm{O}_{12}$<smiles>O=c1[nH]c(=O)c2nccnc2[nH]1</smiles>

Lumazine (257) $\mathrm{C}_{6} \mathrm{H}_{4} \mathrm{~N}_{4} \mathrm{O}_{2}$

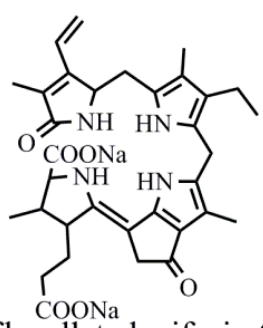

Dinoflagellate luciferin (256) $\mathrm{C}_{33} \mathrm{H}_{38} \mathrm{~N}_{4} \mathrm{Na}_{2} \mathrm{O}_{6}$<smiles>Cc1cc2c(cc1C)N(C)c1[nH]c(=O)[nH]c(=O)c1N2</smiles>

Bacterial luciferin (255) $\mathrm{C}_{17} \mathrm{H}_{21} \mathrm{~N}_{4} \mathrm{O}_{9} \mathrm{P}$<smiles>NC(=O)CN1C(=O)/C(=C/c2ccc(O)cc2)N=C1CNC=O</smiles>

YFP chromophore (258)

Figure 1. Cont. 


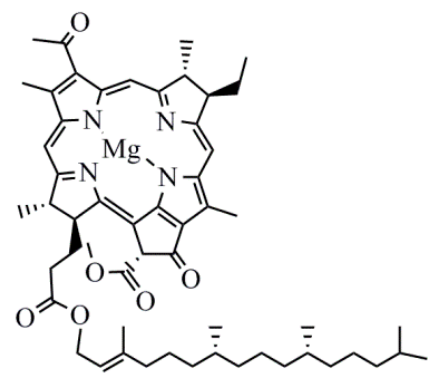

Bacteriochlorophyll (259) $\mathrm{C}_{55} \mathrm{H}_{74} \mathrm{MgN}_{4} \mathrm{O}_{6}$

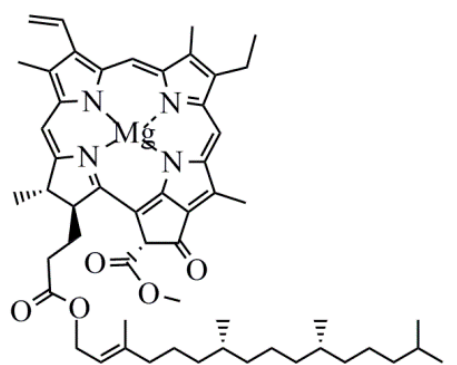

Chlorophyll (260) $\mathrm{C}_{55} \mathrm{H}_{72} \mathrm{MgN}_{4} \mathrm{O}_{5}$

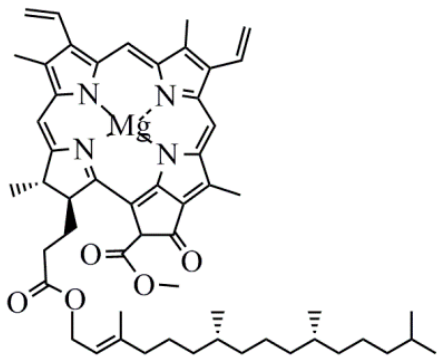

Divinyl chlorophyll (261) $\mathrm{C}_{55} \mathrm{H}_{69} \mathrm{MgN}_{4} \mathrm{O}_{5}$

Figure 1. Chemical structures of various pigments.

\section{Brief Historical Note on Microbial Pigments}

In 1879, a natural yellow pigment called "lactoflavin" was obtained from milk. In 1932, a yellow dye from aqueous yeast extracts was fractioned by Warburg and Christian. Afterwards, Karrer and Kuhn elucidated the yellow pigment called riboflavin, and both of them were endowed with the Nobel Prizes in chemistry for Karrer and Kuhn 1937 and 1938, respectively [22]. In the early 1970s, the purple pigment bacteriorhodopsin of Halobacterium was discovered [23]. Several pigmented non-photosynthetic bacteria and fungi were isolated during 1934 and 1976 by Ingraham and Baumann (both of them had conducted a systematic survey of carotenoid-producing non-photosynthetic bacteria in the 1930s) and Valadon respectively [24]. Monascus pigments are the wello known natural food colorants known around the world since 1884 [25]. In Asia, for more than 10 decades, monascus red pigments appear to be used as food colorants to red pot-roast lamb and red rice koji [21]. So far, a total of 65 different monascus pigmented compounds have been reported, and some of which possessing antimicrobial, anticancer, and anti-obesity activities were recently well reviewed [26]. In 1934, ZoBell and Feltham found that $69.4 \%$ of bacterial colonies grown on agar medium inoculated with seawater and marine sediment were chromogenic. An infallible literature summary carried out by ZoBell in 1946 shows that many of marine bacterial species which spoil fish appeared to be pigmented [27]. Zeaxanthin producing Flavobacterium was isolated during the mid-1960s by scientists at Hoffmann-La Roche [21]. In 1964, thermorubin, a red pigment, was first isolated from a mildly thermophilic soil actinomycetes Thermoactinomyces antibioticus [28].

\section{Host Pigmented Compounds Said to Be of Microbial Origin}

Dolastatin, a well-known antitumor compound isolated from different marine invertebrate species like sea hares and molluscs, has recently been found to actually have originated from their symbiotically associated marine cyanobacteria [13]. Pigments produced by some marine plants, invertebrates, and vertebrates such as seagrass, sponges, corals, molluscs, and tunicates are indeed produced by their epibiotic bacteria [29]. Some of the compounds such as Tambajamine, a yellow pigment molecule isolated from sponges and bryozoans, are believed to originate from endobiotic or epibiotic Pseudoalteromonas [30]. Tambjamines isolated from bryozoans (Bugula dentata and Sessibugula translucens), nudibranchs, and ascidians (Atapozoa sp.) have been found to be produce by Streptomyces sp., Pseudoalteromonas tunicate, and Serratia marcescens [31,32].

\section{Ecology and Habitats of Pigmented Microorganisms}

A plethora of research articles have reported the isolation of pigmented microorganisms like bacteria, fungi, and yeast from terrestrial as well as marine milieus. They are distributed in different geographical conditions, from polar regions to tropical environments and from aerial to deep-sea regions. It is believed that microorganisms from different geographical regions are known to tolerate harsh conditions by producing pigments. Some of the pigmented microbes such as bacteria (e.g., Stenotrophomonas) and yeast (e.g., Rhodotorula) from terrestrial environment are found to enter 
coastal environments through discharges from hospitals and domestic sewages, thereby adapting to marine environment. Literature survey indicates that pigmented bacteria could be divided into two categories of true marine pigmented bacteria-primarily of marine origin and adaptive pigmented bacteria-originated from terrestrial ecosystem and survive and proliferate in coastal environment (Figure 1). Irrespective of the common occurrence of PB in terrestrial environment, marine pigmented microbes are gaining more attention due to their varied bioactive pigment compounds.

Recent studies have been diverted to investigate marine microbial pigments as novel chromogenic compounds for biotechnological and industrial application. The occurrence of PB in a marine environment is found to vary according to geographical and nutritional conditions. Apparently, the diversity of pigmented heterotrophic bacteria (PHB) is less in abundance when compared to the enormous diversity of marine heterotrophic bacteria (MHB). Green and blue pigments are rare colors produced by microorganisms. The colony forming units (CFU) of PHB may vary depending on sampling site, seasonal variation, and availability of nutrients. Occurrence of high frequency of pigmented bacteria is noticed in air-water interfaces [33], glaciers [34], ice cores [35], bacterioneuston (sea surface microlayer) and underlying waters [36], salt lakes [37], deepsea hydrothermal vents [38], and abyssal hot springs (e.g., Thermus). Recently, various pigmented bacterial communities have been isolated from lava caves [39]. P. aeruginosa, a pigmented bacterium, has been reported to isolate from the wounds skin of humans and animals. These PBs are reported to be isolated from different marine niches such as seawater, marine sediment, seagrass, sponge, mussel, sea cucumber [40], algal mats, corals, freshwater, athalassohaline lagoon, marine solar saltern, microbial mats in Antarctic lakes, oil contaminated soil, nonsaline alkaline groundwater, and sea ice (e.g., Algoriphagus) [41] (Figure 2).

Several microbes are noticed exhibiting polyextremophilic characteristics according to their environments, for instance, xerophilic (Penicillium purpurogenum) [42], dimorphic (Metschnikowia laotica), pleomorphic (Arthrobacter), extreme halophilic (Salinibacter), thermophilic (Thermus), psychrophilic (Kocuria polaris), acidophilic (Acidobacterium), alkaliphilic (Microbacterium arborescens), radioresistance (Deinococcus grandis), polyextremophile (Halorubrum), barophilic or piezophilic (Halomonas salaria), and color mimic (Cellulophaga lytica). Various species of microalgae distributed in different environments are also reviewed by different authors [43-48]. Factors driving the limited dispersal of these microorganisms in their respective environment are poorly understood.

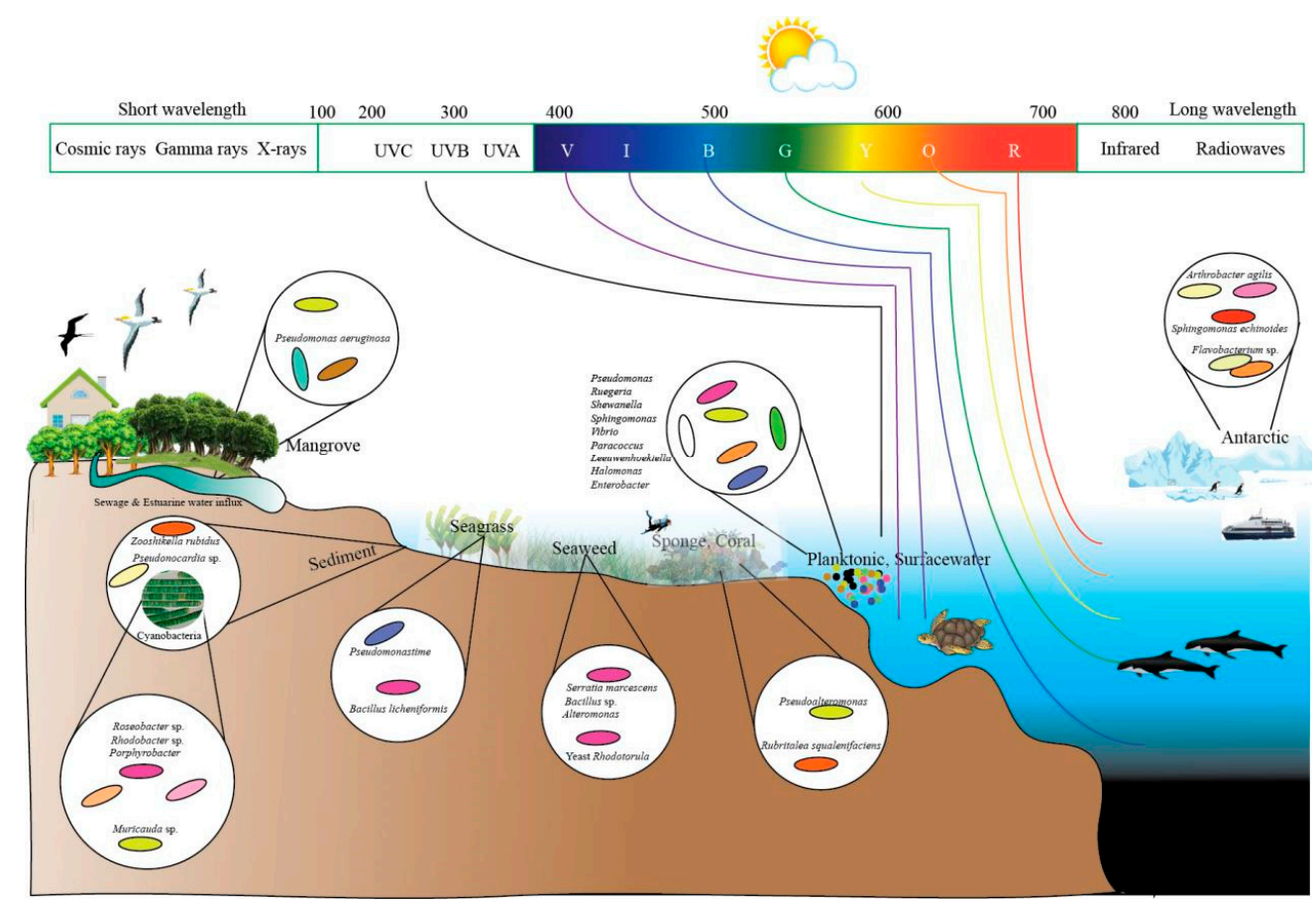

Figure 2. Distribution of marine pigmented microorganisms in different niches. 


\section{Uses of Microbial Pigments}

\subsection{Biological Significance}

Empirically, it is well-understood that most of the microbial pigments found as variety of hues are known to act as defensive systems against UV irradiation, thereby protecting and increasing their survivability [36] and adapting to the surrounding environmental conditions [49] compared to nonpigmented microbes. Carotenoid pigmentation in Antarctic heterotrophic bacteria withstand environmental stresses by adaptation to cold environments [50]. Symbiotic or epibiotic association of some bioactive pigmented bacteria with their host organisms indicates their defensive role in protecting their host from other pathogenic microorganisms and predatory fouling organisms [51]. It was investigated that C50-carotenoids produced by the extremophile microorganisms Halococcus morrhuae, Halobacterium salinarium, and Thermus filiformis are known to be important for their survival as these pigments stabilize their cell membrane and also act as antioxidant agents [52]. Toxic oxygen molecules such as reactive nitrogen species, reactive oxygen species, and other nonbiological radicals formed in the cells are efficiently reduced by carotenoid [52,53]. A violet compound, violacein produced by Chromobacterium violaceum, has been reported to protect lipid membranes such as rat liver microsomes and egg and soy-bean phosphathidylcholine liposomes against peroxidation induced by reactive hydroxyl radicals [54]. Bacterial phenazines are known to regulate cellular gene expressions that trigger the survival and biofilm formation by the bacteria [55]. It is also hypothesized that Thermus strains in natural thermal areas exposed to sunlight are protected by yellow pigmentation [56].

Unambiguously, it was evident that prodigiosin producing Vibrio strains have survived under UV exposure $\left(324 \mathrm{~J} / \mathrm{m}^{2}\right)$ around 1000-fold more successfully as compared to non-pigment-producing vibrios [57]. Bacterial melanins are known to act as cellular protectors by neutralizing diverse toxic chemical compounds like drugs and antibiotics [58] and are one of the survival fitness factors to tolerate stressful physiological conditions like hyperosmotic stress, starvation, and high temperature as observed with Vibrio cholerae [59]. Self-survival defensive mechanism in Janthinobacterium lividum and Chromobacterium violaceum has been related to violacein pigments which have caused cell death to common bacterivorous nanoflagellates Ochromonas sp., Spumella sp., and Bodo saltans when fed on them [60]. The beneficial roles of bacterial pigments are also perceived as protection from phagocytosis. Similarly, indigoidine, a blue quinine compound produced by some Roseobacter strains, annihilates other potentially out competing bacteria; therefore, Roseobacter survives in the environment [2]. Pyoverdin produced by $P$. fluorescens was presumed to have a role in facilitating iron transport as well [61].

Investigations also revealed that marine pigmented bacteria are more resistant to heavy metals and antibiotics compared to nonpigmented bacteria [62]. Melanins in Rhizobium species were found to be involved in the detoxification of polyphenolic compounds accumulated in senescing nodules [63]. Fungal melanins are known to protect fungi from UV and solar radiation (photodestructive impact) and to also inhibit cell-wall-degrading enzymes produced by other microorganisms. Anthraquinones produced by endophytic fungi are found to protect the host plant from insects or other microorganisms [64]. Tambjamines produced by diverse organisms are referred to as natural defensive compounds against predators [31]. Photosynthetic bacteria possess bacteriochlorophylls, bacteriorhodopsins, and proteorhodopsins, which are similar to chlorophylls. Bacteriorhodopsins are light harvesting membrane proteins that enable bacteria to obtain energy when a low amount of organic matter occurs, while halorhodopsin serves as an inward-directed chloride pump and proteorhodopsin serves as a proton pump [65]. In brief, microbial pigments are known to play important roles in different ways including antioxidant activities [54], photosynthesis, cell signaling communication, radiation protection [66], UV absorption [67], antibiotic activities [68], virulence [69], and membrane stabilization [70]. Pigment trait is also used as biological markers for taxonomic identification and the discrimination of different microbes [70]. Interestingly, Claviceps purpurea, an aposamatic fungus, displays a wide range of colors, i.e., yellow, orange, red, and black as warning sign to the predators [71]. 


\subsection{Industrial Significance}

Microbial pigments, especially bacterial pigments are getting more attention due to their wide application in textiles dyeing, cosmetics, food colorants, painting, pharmaceuticals, plastics, etc., and it was assumed that bacterial pigments are to dominate the pigment industries and organic market in near future. Considerably, consumer demand on food grade of important natural microbial pigments such as $\beta$-carotene, riboflavin and phycocyanin is increasing in niche markets [72]. These pigments in the foods serve as preservatives and antioxidants [73]. Synthetic colorants are also employed but are found to cause sickness, so natural pigments are highly preferred over the use of synthetic pigments. Carotenoids obtained from Haematococcus pluvialis and Phaffia rhodozyma are being utilized in pharmaceutical, food additives for animals and fish, and aquaculture industries [65]. Astaxanthins from $H$. pluvialis are also used in aquaculture feeds by aquaculture industries and appear to play a role in memory improvement and antiaging [74]. Xanthan gum, a well-known exopolysaccharide produced by Xanthomonas campestris, is being used as a food additive. Phycocyanin from cyanobacteria is rich in proteins and hence used as dietary supplement; Riboflavin from Bacillus subtilis is used in foods, vitamin enriched milk products, and energy drinks; flexirubin produced by Chryseobacterium and Flavobacterium are used in the treatment of chronic skin disease, eczemea, gastric ulcers, etc.; and bacterial pigments as an indicators of oils spill and as biosensors and markers of water, soil, and air pollution are also known [72]. Several biomedical applications of microbial pigments are detailed in the Supplementary Materials (Table S1).

Cyanobacteria possess chlorophyll "a" and also other pigments like carotenoids, the blue phycobiliproteins, phycocyanin, and allophycocyanin which are potential antioxidants. Strains of Anabaena, Nostoc, and Spirulina are consumed as human food in many countries, and Arthrospira platensisis is marketed in the form of flakes, powder, tablets, and capsules [75]. In Japan, cyanobacterial pigments such as phycocyanin and phycoerythrin are being used for coloring candy, ice cream, yogurt, dairy products, and soft drinks. Phycocyanin obtained from Spirulina is being used in preparing bio-lipsticks (e.g., red pigment from Haematococcus), bio-eyeliners, bio-eye shadows, creams, and soaps. Phycocyanin and phycoerythrins of Spirulina are also being used in fluorescent microscopy, in immunoassays, and as phycofluoures for DNA probes [76,77]. Application of carotenoids as coloring agents for cooked sausages (e.g., bologna and frankfurters), soft drinks (e.g., cola), and baked goods (e.g., Livarot cheeses) have been investigated [78]. Azaphilone pigments and Arpink red ${ }^{\mathrm{TM}}$ (Natu-ral Red $^{\mathrm{TM}}$ ) obtained from Monascus species and Penicillium oxalicum, respectively, have wide applications as red food colorants [21]. Microalgal pigments are also widely being used in aquaculture application, cosmetics, creams, jellies, etc. [79].

A survey by the Infectious Disease Society of America (IDSA) has raised alarm on the urgent threat of antibiotic resistant microbial pathogens, and the WHO has undertaken a project to develop a list of global R\&D priorities with respect to drug-resistant infectious microorganisms. Succinctly, here, the therapeutic uses of different microbial pigmented compounds are described below, which may be highly appreciable to use against various diseases including drug resistant microorganisms and cancer cells.

\subsubsection{Antibacterial Activity}

Prodiginine compounds like prodigiosin, undecylprodigiosin, cycloprodigiosin, heptylprodigiosin, nonylprodigiosin, cyclononylprodigiosin, and cyclomethyl-decylprodigiosins are well-known to exhibit various biological properties including antibacterial activities against different gram-negative and gram-positive bacterial members [80]. Tambjamines and other members of this class compounds are produced by marine bacteria like the well-known Pseudoalteromonas tunicata and possess a wide range of antibacterial activities [49]. Violacein extracted from Janthinobacterium lividum and Chromobacterium violaceum exhibited a wide range of antibacterial activity against gram-positive and gram-negative bacteria [81]. Tetrabromopyrrole, the yellow pigment extracted from Chromobacterium, a seawater isolate, was known to inhibit different human pathogens as well as marine bacteria including 
autoinhibition of the producing bacteria [82]. Several other phenazine compounds [83], quinones [63], and anthroquinones biosynthesized by different bacterial and fungal species also showed a broad range of antibacterial activities [64].

\subsubsection{Antifungal Activity}

Prodiginines such as prodigiosin, undecylprodigiosin, and cycloprodigiosin compounds have contributed as fungicidal agents against several fungi such as Coccidioides, Candida, Didymella, Aspergillus, Penicillium, Saccharomyces, Cryptococcus, Histoplasma, Trichophyton, and Verticillium [80]. Tambjamines are yellow pigments and are believed to be originated from bacterial species such as Pseudoaltermonas tunicata and to possess antifungal properties [30]. Fungicidal activity of violacein isolated from Janthinobacterium lividum has also been reported against white root rot causing phytopathogenic fungi Rosellinia necatrix [84]. Some anthraquinone compounds synthesized by Trichoderma harzianum, Curvularia lunata [64] and phenazine compounds synthesized by Pseudomonas and Streptomyces species are also demonstrated to have antifungal activities against various fungal species [83].

\subsubsection{Antiviral Activity}

Phenazine compounds synthesized by Pseudomonas and Streptomyces species have been reported to show promising antiviral activities [83]. Violacein demonstrated a significant level of antiviral activities against herpes simplex virus, poliovirus, and simian rotavirus SA II [81]. Quinone compounds such as benzoquinones, naphthoquinones, and anthraquinones are well-known to demonstrate antiviral properties $[64,85]$.

\subsubsection{Antimetastatic Activity}

In vitro and in vivo investigations on the antimetastatic activity of prodigiosin revealed the inhibition of metastatic nodules of human highly metastatic lung carcinoma 95-D cells and the highly metastatic substrain B16BL6 of mouse melanoma B16 cells. Results also showed the elevated survival rate of mice, indicating the potentiality of prodigiosin as an antimetastatic compound that is to be focused for further research [86].

\subsubsection{Immunosuppressive Activity}

Intriguingly, cycloprodigiosin hydrochloride, a red pigment obtained from Pseudoalteromonas denitrificans, is stable under several physicochemical conditions and demonstrated immunosuppressive activity by inhibiting the proliferation of T cells and PMA (Phorbol 12-myristate 13-acetate) stimulated Jurkat cells [87]. At nontoxic concentrations, prodigiosin inhibited the T-cell mediated immune functions such as concanavalin-A induced proliferation, mixed lymphocyte response, local graft vs. host reaction, and T-dependent antibody response [88]. Undecylprodigiosin demonstrated the inhibition of purified peripheral human T and B lymphocytes with an $\mathrm{IC}_{50}$ of 3 to $8 \mathrm{ng} / \mathrm{mL}$ and elicited the inhibition of retinoblastoma protein phosphorylation by inhibiting cyclin-dependent kinase- 2 and cyclin-dependent kinase-4 in human lymphocytes [89]. Tambjamine alakaloids and its various other related members are found to possess immunosuppressive activities [49].

\subsubsection{Antitumor Activity}

A well-detailed recent review by Soliev and Enomoto [13] corroborated that several pigmented compounds belong to structural classes of polyketide, pyrroloiminoquinone, indolocarbazole, butenolide, phenoxazinone, alkaloid, phycobiliprotein, terpenoid dihydroquinones, phenazine, peptides, indole, and pyrrole alkaloid known to be produced by marine Pseudoalteromonas, marine Actinomycetes, marine cyanobacterial species, and other bacterial species with potential antitumor activities. Numerous marine- and terrestrial-derived fungal species produce pigmented anthroquinone compounds, which have potential in inhibiting tumor cells [90]. 


\subsubsection{Anti-Alzhelmeric Activity}

Phycobilioproteins originating from red algae and cyanobacterial species are shown to display anti-alzhelmeric activity [77].

\subsubsection{Antiatherosclerosis Activity}

The repression of lipid peroxidation and atherosclerotic plaque by Monascus-fermented red mold dioscorea (RMD) including a higher monacolin $\mathrm{K}$ level and a dioscorea substrate have contributed to potent anti-atherosclerotic effects with $48 \mathrm{mg} / \mathrm{kg} /$ day [91].

\subsubsection{Antihypertensive Activity}

Significantly, the oral administration of monascus-fermented dioscorea at a low-dose $(150 \mathrm{mg} / \mathrm{kg})$ in spontaneously hypertensive rats (SHRs) has revealed decreased systolic and diastolic blood pressures [92]. The antihypertensive activity had earlier been reported from the microalgae Dunaliella tertiolecta [93].

\subsubsection{Anticancer Activity or Antineoplastic Activity}

Prodigiosin pigments produced by Serratia marcescens have induced apoptosis in haematopoietic cancer cell lines and human colon cancer cells activities [94]. Quinones are yellow to red compounds that demonstrated significant anticancer activities [63]. Significantly, violacein extracted from C. violaceum showed cytotoxic effects and apoptosis of different cancer cells including colorectal cancer, uveal melanoma, leukemia, and lymphoma cells in culture [69]. A yellow pigment producing Pseudoalteromonas piscicida strain NJ6-3-1 isolate obtained from sponge Hymeniacidon perleve possesses cytotoxic activity on cancer cells HeLa or BGC-823 cell lines, with $\mathrm{IC}_{50}$ values of $150 \pm 4.6$ and $192 \pm 3.5 \mu \mathrm{g} / \mathrm{mL}$, respectively [95]. Bacterial phenazine compounds also appeared to be potential anticancer agents [96]. Monascus pigments such as monascin, ankaflavin, monaphilone A and monaphilone $\mathrm{B}$, monascuspiloin, monascorubrin, rubropunctatin, and monascorubramine exhibited significant cytotoxic activities against various cancer cell lines [26]. $\beta$-carotene synthesized from microalgal species have been found to be a potential anticancer agent in human and animal model studies [79]. Phycobilioproteins produced by different cyanobacterial species and red algae are also known to be anticancer agents [77].

\subsubsection{Anti-Tuberculosis Activity}

Violacein and flexirubin pigments isolated from Antarctic bacteria Janthinobacterium sp. Ant5-2 and Flavobacterium sp. Ant342 demonstrated the growth inhibition of Mycobacterium tuberculosis with minimum inhibitory concentrations (MICs) of 34.4 and $10.8 \mu \mathrm{g} / \mathrm{mL}$ for virulent $M$. tuberculosis, respectively [97].

\subsubsection{Antifouling Activity}

Pigmented Pseudoalteromonas bacterial species isolated from marine plants and animals appeared to be effective inhibitors against common fouling organisms such as invertebrate larvae of Hydroides elegans and Balanus amphitrite, algal spores of Ulva lactuca and Polysiphonia sp., diatoms, bacteria, and fungi [98]. The same inhibitory activity was recently reported from prodigiosin producing $S$. marcescens CMST07 that inhibited marine fouling bacteria like Alteromonas sp. and Gallionella sp. with a minimum inhibitory concentration (MIC) and a minimum bactericidal concentration (MBC) of 6.75 and $12.5 \mu \mathrm{g} / \mathrm{mL}$, respectively [99]. Also, Prodigiosin was found to inhibit cyanobacterial adhesion on glass surfaces [99]. Investigations have also reported the correlation between pigmentation and antifouling activities $[51,98]$. 


\subsubsection{Anti-Algicidal Activity}

Serendipitously, purified prodigiosin extracted from Hahella chejuensis, a marine bacterium, showed complete inhibition of algicidal activity against a major red-tide dinoflagellate Cochlodinium polykrikoides at a $10^{-1} \mathrm{mg} / \mathrm{L}$ concentration [100] or at low concentrations as $\sim 1 \mathrm{ppb}$ [101]. Xylindein, a blue-green compound from a fungal species Chlorociboria aeruginosa, was found to control the Chlorella growth [102].

\subsubsection{Anti-Insecticidal Activity}

Naphthoquinone pigments such as fusarubin, javanicin, and related compounds are reported to display insecticidal activities [63,64].

\subsubsection{Anti-Herbicidal Activity}

Anthraquinones from several fungal species such as Phoma exigua var. exigua, Phoma foveata, P. glomerata, P. herbarum, P. macdonaldii, P. macrostoma, P. multirostrata, P. proboscis, P. sorghina, and P. tracheiphila possess herbicidal activities [103]. Quereshi et al. (2011) [104] isolated a pigment compound-anhydropseudophlegmacin-9,10-quinone- $3^{\prime}$-amino-8'-O-methyl ether-from Phoma herbarum FGCC\#54 that showed potential herbicidal activity against prominent weeds Hyptis suaveolens, Lantana camara, Parthenium hysterophorus, and Sida acuta.

\subsubsection{Antiparasitic Activity}

The violacein pigment compound obtained from Chromobacterium violaceum has exhibited in vitro antiparasitic activity as trypanocide activity by the growth inhibition of Trypanosoma cruzi [105]. Lopes et al. (2009) [106] reported the inhibition of chloroquine-sensitive and -resistant strains of Plasmodium falciparum by violacein with an $\mathrm{IC}_{50}$ value of $0.85 \pm 0.11 \mu \mathrm{M}$.

\subsubsection{Antiprotozoal Activity}

Violacein extracted from freshwater isolates of Janthinobacterium lividum and Chromobacterium violaceum is reported to have protozoan-killing efficiency against cultures of nanoflagellates Spumella sp. and Ochromonas sp. At higher concentrations of $>10 \mu \mathrm{M}$, it resulted in the complete reduction of the cells, indicating their defensive role in avoiding being ingested by these nanoflagellates [60]. Antiprotozoal activities by red pigment prodigiosin have also been reported from Serratia [107].

\subsubsection{Antileishmanial Activity}

Reduction in viability/growth inhibition of Leishmania amazonensis with violacein compound was observed at the concentration of $\mathrm{EC}_{50} / 24 \mathrm{~h}$ value of $4.3 \pm 1.15 \mu \mathrm{mol} / \mathrm{L}$ [108]. Prodigiosin producing S. marcescens variant SM 365 has evidently contributed to the lysis of Leishmania chagasi [109].

\subsubsection{Antiulcerogenic Activity}

Violacein, a purple violet pigment, has demonstrated increased inhibition of gastric damage (ulcer formation) in the presence of $\beta$-cyclodextrin $(\beta C D)$ inclusion complexation at 1:1 and 1:2 molar ratios compared to that of violacein [110]. Flexirubin pigments from Chryseobacterium and Flavobacterium are used in treatment for chronic skin disease, eczemea, gastric ulcers, etc. [8,72].

\subsubsection{Antilipoperoxidant Activity}

Violacein and $\beta C D$ inclusion complexation at 1:2 ratio exhibited four-fold potent antilipoperoxidant activity compared to violacein in rat liver cells by a $40 \%$ inhibition of malonaldehyde (MAD) with an $\mathrm{IC}_{50}$ of 125 and $505 \mu \mathrm{M}$ [110]. 


\subsubsection{Anti-HIV Activity}

Compounds extracted from pigmented Phoma species have demonstrated inhibition of HIV virus integrase [103]. In vitro investigations were also initiated to evaluate the effect of violacein on AIDS-related lumphoma [111].

\subsubsection{Anti-Malarial Activity}

Violacein, a violet pigment extracted from Chromobacterium violaceum is known to exhibit more effective antimalarial activity against Plasmodium falciparum strains in vitro [106]. Prodiginines such as cycloprodigiosin, prodigiosin, undecylprodigiosin, heptyl prodigiosin, and metacycloprodigiosin have demonstrated the antimalarial activity against $P$. falciparum $[80,112,113]$. Liu et al. (1993) [114] reported the mosquitocidal activity of melanin produced by Bacillus thuringiensis subsp. israelensis.

\subsubsection{Antitrypanosomal Activity}

Recently, Genes et al. [115] reported that prodigiosin extracted from S. marcescens have apparently appeared to cause cell death of Trypanosoma cruzi by disrupting the mitochondrial function and by interfering with the oxidative phosphorylation processes. In vitro experiments also demonstrated that merely prodigiosin producing S. marcescens has resulted in cell lysis of trypanosomatid protozoan parasites T. cruzi $[81,116]$.

\subsubsection{Antinematodal Activity}

Phenazine compounds extracted from Pseudomonas fluorescens are found to suppress egg hatching and to enhance the juvenile mortality of root knot nematode, Meloidogyne incognita, in vitro [117].

\subsubsection{Anti-Inflammatory Activity}

Investigations on red mold dioscorea (RMD) have demonstrated the anti-inflammatory effects in STZ-induced diabetic rats by reducing inflammatory cytokine TNF- $\alpha$ levels and enhancing IL-2 cytokine expression [118]. Monascin, a yellow metabolite of monascus, displayed anti-inflammatory activity by inhibiting inflammatory the signal pathways of PKC (protein kinase C) and JNK (c-Jun N-terminal kinase) phosphorylation in a C2C12 cell model [119].

\subsubsection{Antihypertriglyceridemia Activity}

A study corroborated that increased levels of high-density lipoprotein and decreased levels of triglycerides (TG) and glycosylated hemoglobin (HbA1c) in DM (diabetes mellitus) + 1X RMD and $\mathrm{DM}+5 \mathrm{X}$ RMD supplemented rats displayed antihypertriglyceridemia activity [118].

\subsubsection{Anti-Atherosclerotic Activity}

Ankaflavin and monascin are proven to prevent the accumulation of fatty liver and lipid plaque and enhanced high-density lipoprotein cholesterol, respectively, in heart aorta of hamsters [120]. It was concluded that ankaflavin also acts as a potential hypolipidemic agent [120]. Astaxanthin from different microbial sources has been reported to exert preventive actions against atherosclerotic cardiovascular disease by the enhancement of oxidative stress, inflammation, lipid metabolism, and glucose metabolism [121].

\subsubsection{Antioxidant Activity}

Regardless of common carotenoids like lutein, $\beta$-carotene, astaxanthin, etc., the antioxidnat activity of rare $\mathrm{C}_{50}$ carotenoids such as sarcinaxanthin, sarcinaxanthin monoglucoside, and sarcinaxanthin diglucoside with $\mathrm{IC}_{50}$ values of 57,54 , and $74 \mu \mathrm{M}$, respectively, were reported from a halophilic bacterium Micrococcus yunnanensis strain AOY-1 isolated from hard coral [122]. Violacein is a strong antioxidant compound that can protect lipid membranes from peroxidation caused by hydroxyl 
radicals [49,54]. Monascus pigments are reported to act as effective antioxidants [26]. Carotenoids with both large numbers of conjugated double bounds and of hydroxyl groups appeared to have strong antioxidant activity. Mandelli et al. [52] reported the antioxidant activity demonstrated by extremophile microorganisms Halococcus morrhuae $\left(\mathrm{IC}_{50}=0.85 \mu \mathrm{g} \cdot \mathrm{mL}^{-1}\right)$, Halobacterium salinarium $\left(\mathrm{IC}_{50}=0.84 \mu \mathrm{g} \cdot \mathrm{mL}^{-1}\right)$, and Thermus filiformis $\left(\mathrm{IC}_{50}=2.41 \mu \mathrm{g} \cdot \mathrm{mL}^{-1}\right)$. A structurally unusual phenolic carotenoid, 3,3'-dihydroxyisorenieratene isolated from the bacterium Streptomyces mediolani [123], phycobiloproteins from cyanobacterial species, and some algal species have demonstrated powerful antioxidant activity [77]. Cyanobacterial pigments such as $\beta$-carotene, lycopene, lutein C-phycocyanin, and phycobilioproteins are known to demonstrate antioxidant properties [77].

\subsubsection{Anti-Proliferation Activity}

Undecylprodigiosin also acts as an anti-proliferative agent against human $\mathrm{T}$ and $\mathrm{B}$ lymphocytes with an $\mathrm{IC}_{50}$ value of 3 to $8 \mathrm{ng} / \mathrm{mL}$ [89]. Astaxanthin from the yeast Phaffia rhodozyma demonstrated the antiproliferative activity on MCF-7 and MDA-MB231cell lines [124]. Tambjamines [49] and beta-carotene [125] are also reported to possess anti-proliferation activities.

\subsubsection{Anti-Aging Activity}

Natural astaxanthin pigments appeared to be potential anti-aging supplements [74]. Also, water-soluble phycobilioproteins biosynthesized from cyanobacteria and red algae are found to show potential anti-aging activities [77].

\subsubsection{Anti-Obesity Activity}

L-Tryptophan and L-leucine ethyl ester derivatives of the monascus pigments are GRAS (generally recognized as safe) compounds showing an anti-obesity effect on mice by inhibiting cholesterol and triglyceride contents [126]. Monascin and ankaflavin have reduced the preadipocyte proliferation of 3T3-L1 cells at a $8-\mu \mathrm{g} / \mathrm{mL}$ concentration; decreased the triglyceride accumulation; and suppressed the expression of adipocyte specific transcription factors, C/EBP $\beta, \mathrm{C} / \mathrm{EBP} \delta, \operatorname{PPAR} \gamma$, and C/EBP $\alpha[120,127]$. TEA (2-(p-toyly) ethylamine), an amine derivative of monascus pigment, reduced the total cholesterol (24\%) and LDL (low-density lipoprotein) cholesterol (38\%) content in C57BL/6 mice serum [128]. The red mold dioscorea (RMD) cultured with deep ocean water (DOW-RMD) with increased levels of monascin and ankaflavin have displayed anti-obesity effects by inhibiting PPAR $\gamma$ and C/EBP $\alpha$ expression in differentiation and lipoprotein lipase activity [129].

\subsubsection{Anti-Diabetic Activity}

Monascus fermented red mold dioscorea appeared to delay diabetes by showing antioxidant effects, protection of pancreatic $\beta$-cells, and control of hyperglycemia by decreasing blood glucose and serum-free fatty acid levels in Streptozotocin-induced diabetic rats [118]. Similarly, monascus fermented durian seed (MFDS) ethanol extracts have exhibited potentiality towards diabetes mellitus by $\alpha$-glucosidase inhibitory activity with an $\mathrm{IC}_{50}$ of $70.7 \mu \mathrm{g} / \mathrm{mL}$ [130]. Monascins are found to prevent PPAR- $\gamma$ phosphorylation by phospho c-Jun N-terminal kinase (p-JNK) to exhibit anti-diabetic activity [119].

\subsubsection{Antiadipogenic Activity}

Monascin and ankaflavin also promote delipidation of mature adipocytes by glycerol release by $113.2 \%$ and $278.3 \%$ and reduce the downregulation activity of HR-LPL (heparin-releasable lipoprotein lipase) by $45.3 \%$ and $58.1 \%$, respectively [127]. High fat diet (HFD) supplemented with the amine derivatives of monascus pigments, 4- phenylbutylamine (PBA) $(2.5 \mu \mathrm{M})$, and 2-(p-toyly) ethylamine (TEA) $(12.5 \mu \mathrm{M})$ have demonstrated an inhibitory activity against adipogenic differentiation in 3T3-L1cells [128]. Metals present in deep ocean water are found to cause synergistic effects on the production of monascin and ankaflavin, and DOW with RMD shows a significant anti-adipogenesis effect [131]. 


\subsubsection{Ichthyodeterrent Activity}

New tambjamine compounds isolated from ascidian Atapozoa sp. [132], and bryozoan Bugula dentata [31] appeared to originate from their associated symbiotic bacteria $S$. marcescens and possess Ichthyodeterrent activities.

\subsubsection{Conjugated Antibodies}

Phycoerythrins are widely used in fluorescent probes and have been commercialized as conjugated antibodies [133].

\subsubsection{Cytotoxic Activity}

A marine bacterial strain Pseudoalteromonas maricaloris $\mathrm{KMM} 636^{\mathrm{T}}$ isolated from sponge Fascaplysinopsis reticulata was found to produce two brominated yellow pigments bromoalterochromide $\mathrm{A}$ and $\mathrm{A}^{\prime}$. These compounds displayed a cytotoxic effect on developing eggs of the sea urchin Strongylocentrotus intermedius [134]. Grossart reported the cytotocic effect of deep blue pigment glaukothalin extracted from Rheinheimera strains (isolated from diatom aggregates and organic particles) against Artemia salina ( $c=0.1 \mathrm{mg} / \mathrm{mL}$, mortality $=100 \%)$ [135].

\subsubsection{Inducing Activity as Larval Metamorphosis}

Tetrabromopyrrole isolated from four Pseudoalteromonas bacterial strains have induced metamorphosis of acroporid coral Acropora millepora larvae, i.e., planulae transformation into fully developed polyps within $6 \mathrm{~h}$, indicating that the settlement of these larvae on crustose coralline algae is mediated through epibiotic microbes [136]. Similarly, the enhanced production of eggs and juveniles was observed upon the addition of lutein and zeaxanthin to the adult diet of sea urchin Lytechinus variegatus [137].

\subsubsection{Miscellaneous Activities}

Lutein and zeaxanthin are being used in nutraceutical and as dietary supplements to prevent cardiovascular diseases, cancers, cognitive function, and age-related macular degeneration (AMD) [21]. Evidently, cell free culture filtrates (with presence of 1.09 to $9.89 \mu \mathrm{g} \cdot \mathrm{mL}^{-1}$ of cytokinins) of pink pigmented Methylobacterium strains isolated from the phyllosphere of different crop plants such as sugarcane, pigeonpea, mustard, potato, and radish have enhanced the seed germination of wheat Triticum aestivum [138]. Monascin and rubropunctatin pigments from Monascus purpureus have displayed teratogenic effects on chicken embryos [139]. Phycobilioproteins extracted from cyanobacterial species are being used as fluorescence probes as protein markers for gel electrophoresis [77]. Evidently phycoerythrin-feeding appeared to increase the mean survival percentage of Caenorhabditis elegans [77].

\section{Factors Affecting Pigment Production}

Decades of research prospects on single-cell prokaryotes to multicellular eukaryotic organisms have corroborated that their life cycles depend on a broad range of physicochemical parameters which regulate or hamper the production of various metabolites. Three major routes are known for the production of any kind of microbial metabolites: (1) Naturally produced metabolites; (2) metabolites produced under unfavourable/strained environmental conditions; and (3) metabolites produced upon stimulation with various carbon, nitrogen, and additional nutrient amendments. The literature implies that the production of different pigment molecules are intra- or extracellular (or both) and dependent on light, $\mathrm{pH}$, temperature, and various media constituents [140] and shifts over time and space such as seasonal factors (alluvial, nival, pluvial), sampling sites and habitats, and different cultivation conditions in the laboratories [49].

It is often encountered that microbial pigments, especially bacterial pigmentations, appear is to be ephemeral in nature under laboratory conditions and also when the culture frequently subcultured. However, reverting respective pigmentation may be possible if the culture is supplied with various 
factors such as environmental parameters and the optimization of medium components (Table 1). Empathizing microbial feelings (e.g., nutrient amendments) in an understandable way is always important for better pigment production research (Figure 3).

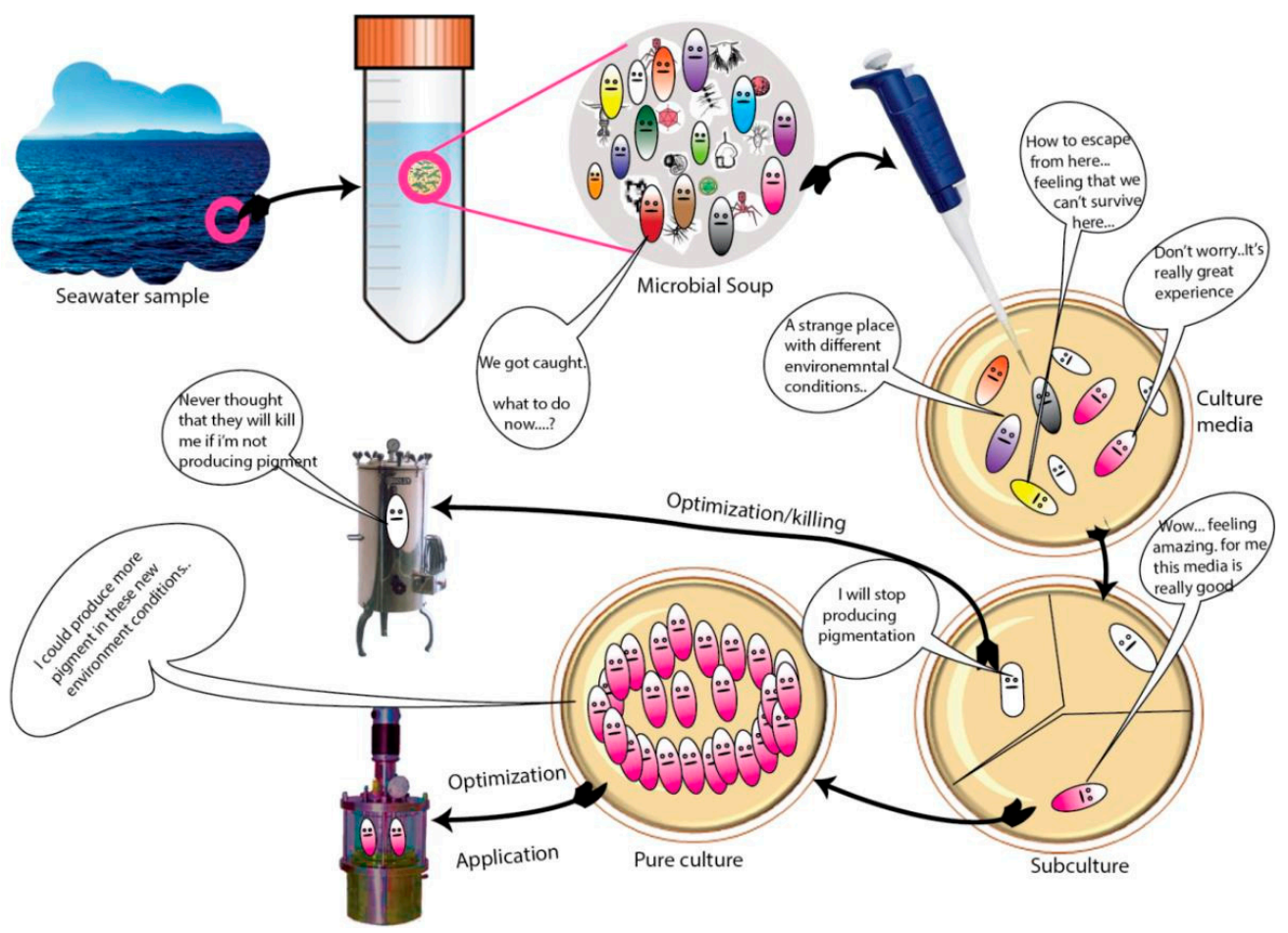

Figure 3. An illustration showing the feelings of microbes.

Nutrient conditions (richness/poorness) always exert a perceptible effect on pigment synthesis, as earlier studies observed that high phosphate content and high acidity cause diminution of fluorescent pigment and that trace amounts of sulphate can vitiate pigment synthesis [141]. Organic acids produced during Monascus ruber culture in oxygen-excess conditions appear to inhibit pigmentation [142]. The addition of several substrates such as rice and wheat meals (either integral or broken residual cereal) and light stimulation have induced high levels of carotenoid production in fungi and yeasts [143]. In 1944, ZoBell and Upham observed that pigmentation was increased when bacteria was grown in sea water enriched with beef extract, bacto-tryptone, and neopeptone at $4{ }^{\circ} \mathrm{C}$. In 1946 , ZoBell indicated that the infusions of marine animals such as fish, octopus, and mussel and other animals stimulated pigment production [27]. A study found that pink pigment production in Acinetobacter wofii was induced by methanol as sole source of carbon [144]. Mycobacterium tuberculosis was found to produce carotenoid pigments in acidic stress at $\mathrm{pH}$ 5.0-6.0 and long-term growth in anaerobic culture conditions [145], and several other Mycobacterium species appeared to produce different pigments [146].

It was studied that a large amount of water-soluble yellow-green fluorescent pigment synthesis by $P$. fluorescens depends on the addition of succinate as the sole carbon source, and no pigment production was observed upon the addition of citric and malic acids as substrates [63]. Carbon sources such as glucose and inorganic nitrogen sources like ammonium sulphate, peptone, or other amino acids also induce pigmentation. Photochromogenic (photoinduction) and scotochromogenic (pigment formation in the dark) effects on pigment synthesis have been found in nontuberculous Mycobacteria [63]. Mycobacterium marinum, Myxococcus xanthus (bacteria), Dacryopinax spathularia (fungus), and Rhodotorula glutinis (yeast) were found to produce carotenoids in the presence of light [63]. Similarly, pigment syntheses by the microalgae appeared to be greatly influence by temperature, salinity, pH, and the light color and intensity [79,147]. 
Observations deduced that many nonpigmented Thermus strains at high growth temperatures produce an unstable and irreversible yellow pigmentation product [56]. Some bacterial cultures for instance, e.g., a well-known marine $S$. marcescens produce red pigment on solid peptone-glycerol agar plates, however, failed to produce pigment in a peptone-glycerol liquid medium. Nevertheless, pigmentation was induced in a liquid medium culture when supplemented with silica gel [148]. Chen et al. revealed that elevated levels of prodigiosin production was perceived when supplemented with starch and peptone as carbon source, and significantly, prodigiosin production was increased from $7.05 \mathrm{~g} / \mathrm{L}$ to $15.6 \mathrm{~g} / \mathrm{L}$ with the addition of calcium alginate beads as a porous carrier [149]. Increase in pigment production in the cells may be seen when subjected to stress conditions such as high temperature, osmotic pressure, metabolic inhibition, and the existence of heavy metals, etc. [72]. Production of a pigment (e.g., glaukothalin) was found to be linked with the presence of acylated homoserine lactones (AHL), amino acids, and other bacterial strains [135]. Violacein pigment synthesis in C. violaceum is regulated by $\mathrm{N}$-acylhomoserine lactone (AHL)-dependent quorum-sensing system [60]. The enhancement of pigment production has been observed upon introduction of mutagens such as UV light, ethyl methane sulfonate, and 1-methyl-3-nitro-1-nitrosoguanidine in Haematococcus pluvialis and similarly microwave on Serratia marcescens [73]. Further, enhanced pigment production from interested microbe may be achieved by altering genes (gene knockout or promotion) or mutagenesis techniques [72]. To envisage microbial pigments for industrial production, evaluating several suitable substrates and physicochemical parameters for interested pigmented microbe is always an essential step to yield a high amount of pigments for various applications. A detailed description has been reviewed on the extraction of various pigmented compounds from microalgae $[9,48,150]$, fungi $[90,103,151]$, bacteria [49], and yeasts [152].

Biosynthesis and expression of pigments in different microorganisms are regulated by respective genes which impart color to the cells. Different substrates such as phenylalanine, tryptophan, and, more significantly, tyrosine were found to be good stimulators for various pigment compounds; however, efforts are to be extended to know other substrates' efficiency in promoting pigmentation (Table 1).

Table 1. Various media and supplements required for extraction of specific pigments from different microorganisms.

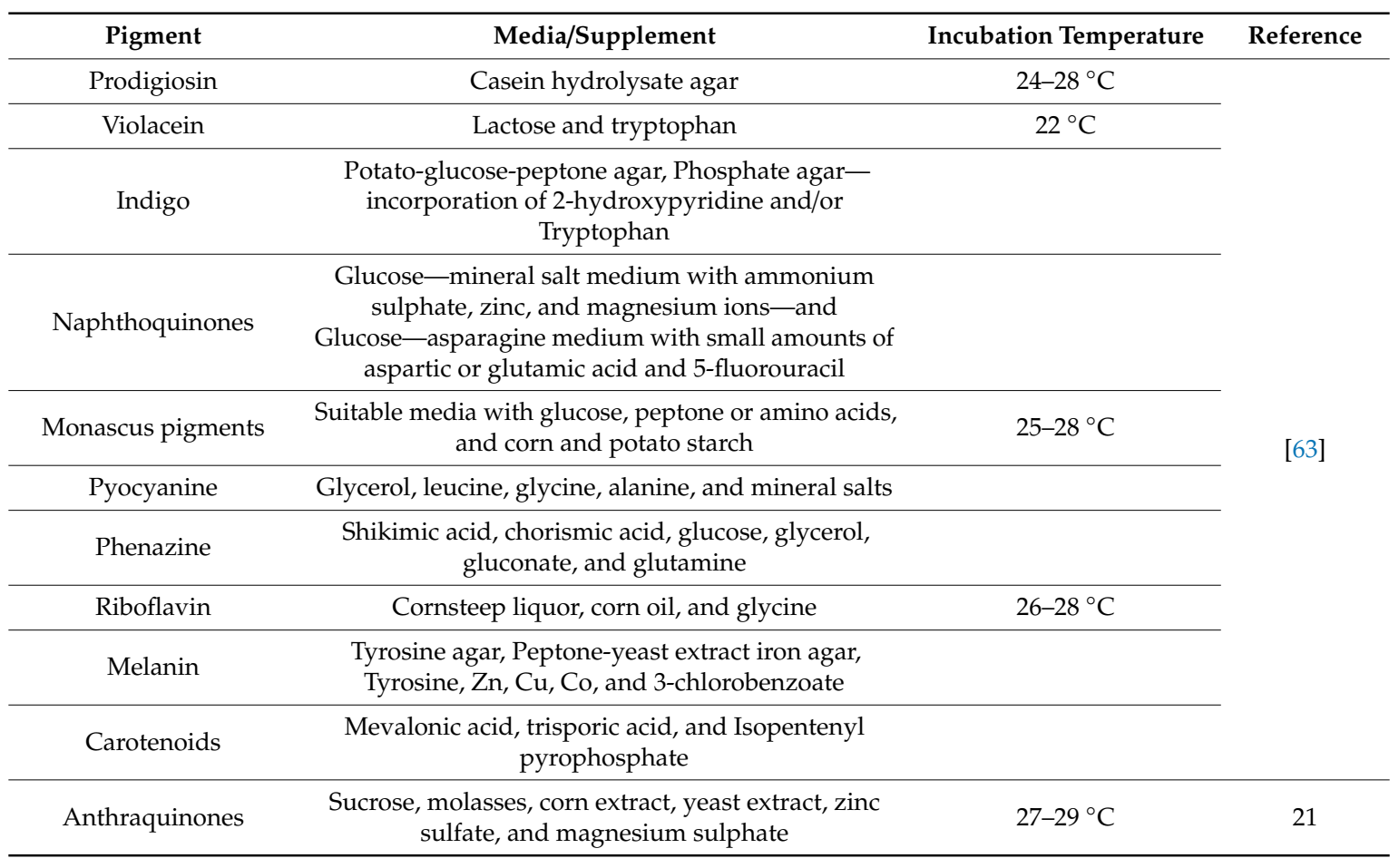




\section{Challenges in Pigment Compound Development}

Irrespective of terrestrial or marine origin, to bring out any kind of versatile therapeutic or nutraceutically important microbial pigment products into the market, a lot of investment along with experimental work (e.g., prior assessment of color stability in heat (thermolabile—various temperatures and autoclaving), light (photolysis), $\mathrm{pH}$, agitation, aeration, dissolved oxygen, etc. are most important concerns to be studied for various biotechnological applications), in addition to solubility (e.g., lipolytic, hydrosoluble, and oxidized), optimization process, extensive toxicological studies (e.g., acute oral toxicity in mice 90-day subchronical toxicological study, acute dermal irritation, acute eye irritation, antitumor activity, micronucleus test in mice, AMES test, estimation of antibiotic activity, and estimation of microbial toxins), regulatory approval (e.g., EU and USA legislations, Codex Alimentarius Commission, Food and Drug Administration, European Food Safety Authority, Pharmaceutical and Food Safety Bureau, and National Agency of Sanitary Vigilance), and penchant by the consumers are highly required $[21,153,154]$.

Other factors to be considered for desired productivity of microbial pigments in fermentation aspects are type of bioreactor and its design (e.g., traditional bioreactors, stirred-tank and air lift reactor, and trickle-bed reactor), type of fermentation (batch, feed-batch, or continuous), and physicochemical and biological conditions in fermentation process [72]. Upon successful achievement of these requirements, there would be potential demand in the biggest markets for food pigments such as Europe and United States [17]. Storage of pigments is suggested to be in the dry powder form or liquid concentrates, with the former being more preferable due to its low water activity and high stability [143].

Many other pigments are to be commercialized; however, commercialization ventures are found to be hampered by cost-effective synthetic medium, which are being alternatively substituted by the utilization of cheap agro-industrial residues as substrates (e.g., corn meal, peanutmeal, coconut residue, soybean meal, rice water, jackfruit seed, tapioca starch, grape juice, grape must, peat extract, mustard waste, liquid pineapple waste, mung bean waste, sugar beet molasses, corn syrup, starch, cheese whey, minerals, and vitamins) to maximize pigment production [72,153]. Enhancement of pigment production may be effective when culture conditions are optimized with several substrates and using via RSM (Response Surface Methodology) combined with the ANN (Artificial Neural Network) statistical approach [72]. Taskin et al. prepared chicken feather peptone (CFP) from waste chicken feathers and found that CFP induces carotenoid and biomass production about 53 and $36 \%$ at $8 \mathrm{~g} / \mathrm{L} \mathrm{CFP}$ concentration respectively [155].

\section{Pathogenicity of Pigmented Microbes}

Regardless of microbial pigments in various applications, some of the pigments produced by certain microorganisms are known to promote pathogenicity and virulence. On sheep blood agar, $P$. aeruginosa is often strongly beta haemolytic and can produce different diffusible pigments such as pyocyanin, a green coloured pigment. Vibrio campbellii has been reported to produce a brown pigment which may be due to pyomelanin [129] or proteorhodopsin [156], and this species is a significant pathogen in harveyi clade. Phenazines produced by pseudomonads are known to play a role in virulence [55]. Virulence and pathogenicity in several species of bacteria (e.g., Vibrio cholerae) and fungi (e.g., Cryptococcus neoformans and Aspergillus fumigatus) for their respective animal or plant hosts appeared to be linked with melanin production. Mycobacterium marinum is known to cause infections on skin and soft tissues [157], and some of the Bacillus species are also reported to be pathogens. Serratia marcesens is a well-known agent of nosocomial infections of the urinary tract and wounds [69]. Other pigmented compounds such as golden staphyloxanthin, porphyrin, and granadaene produced by Staphylococcus aureus, Porphyromonas gingivalis, and Streptococcus agalactiae, respectively, are also related to potential virulence functions [69]. Violacein producing Chromobacterium violaceum is an opportunistic pathogen for animals and humans and can cause fatal septicemia from skin lesions with many liver and lung abscesses [158]. Stenotrophomonas maltophiliais is also an emerging human pathogen that is 
responsible for fatal infections in humans [159], and orange pigmentation in this species has recently been reported [40]. Xanthomonas campestris is a phytopathogenic bacterium which causes diseases in cauliflower, cabbages, and rutabagas. Melanin-like compounds producing Aeromonas salmonicida appear to cause fish furunculosis of salmonids [63]. Pyocyanine, a pigmented exotoxin produced by Pseudomonas aeruginosa causes chronic lung infections, namely cystic fibrosis in patients [160].

Rosellinia necatrix a fungal species was found to infect several plants like Narcissus and mulberry and forms white root rot [158]. Melanin producing yeast Cryptococcus neoformans is evidently more virulent (a neurotropic pathogen) than albino mutants [63]. Pencillium marneffei is found to biosynthesize a mycotoxin called "citrinin" that showed nephrotoxic activity in mammals [161,162]. Talaromyces purpurogenus has been reported to produce mycotoxins such as rubratoxin A and B and luteoskyrin in addition to extrolites that may be toxic if injected intraperitoneally (spiculisporic acid) or in the veins of cats (rugulovasine A and B) [163]. Apparently, naphthoquinones from Fusarium solani have damaged the plasma membrane of plants [63]. Many other fungal pigments, mostly naphthoquinone metabolites, are found to be phytopathogenic to different plants [164].

\section{Conclusions}

Natural pigmented compounds originating from microbial sources like bacteria, fungi, and microalgae are found to be more valuable and demandable over synthetic compounds. Especially, in these days, marine environment is being focused on for the exploration of novel and known natural pigments with a broad range of biological activities due to vast marine resources which are known to harbor several known and novel pigment synthesizing microbes and microalgae. Extending the great exploration on the uncovered samples from new habitats belonging to terrestrial and particularly marine environments would certainly give promising results in finding novel compounds from interested microorganisms. Several pigment microbial species have been reported hitherto, and their biological activities are to be evaluated. Therefore, generating data (biological properties) from uninvestigated microbes as well as novel species are of great importance in understanding their biological activities of pigments to develop novel medicinal compounds for biotechnological applications.

Supplementary Materials: The following are available online at http://www.mdpi.com/2076-2607/7/7/186/s1, Table S1: Pigmented compounds of different microorganisms and their biological properties.

Author Contributions: Conceptualization, original draft preparation, image creation, writing—review and editing were by C.R. N.V.V., R.K., C.K.V., and L.D. performed the writing-review and editing.

Funding: This work was supported and funded by the Science and Engineering Research Board (SERB), New Delhi under the National Postdoctoral Fellowship, PDF/2016/000354 dated on 5th July 2016.

Acknowledgments: C.R. thank the SERB for awarding the National Postdoctoral Fellowship, PDF/2016/000354 dated on 5th July 2016. Also, C.R. thanks NIOT and ANCOST for providing the necessary facilities to carry out the project work.

Conflicts of Interest: The authors declare no conflict of interest. The funders had no role in the design of the study; in the collection, analyses, or interpretation of data; in the writing of the manuscript, or in the decision to publish the results.

\section{References}

1. Aylward, F.O.; Eppley, J.M.; Smith, J.M.; Chavez, F.P.; Scholin, C.A.; DeLong, E.F. Microbial community transcriptional networks are conserved in three domains at ocean basin scales. Proc. Natl. Acad. Sci. USA 2015, 112, 5443-5448. [CrossRef] [PubMed]

2. Soliev, A.B.; Hosokawa, K.; Enomoto, K. Bioactive pigments from marine bacteria: Applications and physiological roles. Evid.-Based Complement. Altern. Med. 2011, 2011, 670349. [CrossRef] [PubMed]

3. Venil, C.K.; Zakaria, Z.A.; Ahmad, W.A. Bacterial pigments and their applications. Process Biochem. 2013, 48, 1065-1079. [CrossRef]

4. Narsing Rao, M.P.; Xiao, M.; Li, W.-J. Fungal and Bacterial Pigments: Secondary Metabolites with WideApplications. Front. Microbiol. 2017, 8, 1113. [CrossRef] [PubMed] 
5. Numan, M.; Bashir, S.; Mumtaz, R.; Tayyab, S.; Rehman, N.U.; Khan, A.L.; Shinwari, Z.K.; Al-Harrasi, A. Therapeutic applications of bacterial pigments: A review of current status and future opportunities. 3 Biotech 2018, 8, 207. [CrossRef] [PubMed]

6. Mumtaz, R.; Bashir, S.; Numan, M.; ShinwarI, Z.K.; Ali, M. Pigments from Soil Bacteria and Their Therapeutic Properties: A Mini Review. Curr. Microbiol. 2018. [CrossRef] [PubMed]

7. Ramesh, C.H.; Vinithkumar, N.V.; Kirubagaran, R. Marine pigmented bacteria: A prospective source of antibacterial compounds. J. Nat. Sci. Biol. Med. 2019, in press.

8. Kim, S. Marine Biomaterials: Characterization, Isolation and Applications; CRC Press: New York, NY, USA, 2013; pp. 1-787.

9. Amaro, H.M.; Sousa-Pinto, I.; Malcata, F.X.; Guedes, A.C. Microalgae as a source of pigments extraction and purification methods. In Marine Microorganisms Extraction and Analysis of Bioactive Compounds; Leo, M.L.N., Ed.; CRC Press: Boca Raton, FL, USA, 2016; pp. 99-128.

10. Blunt, J.W.; Copp, B.R.; Keyzers, R.A.; Munro, M.H.G.; Prinsep, M.R. Marine natural products. Nat. Prod. Rep. 2014, 31, 160-258. [CrossRef]

11. Kirti, K.; Amita, S.; Priti, S.; Kumar, A.M.; Jyoti, S. Colorful World of Microbes: Carotenoids and Their Applications. Adv. Biol. 2014, 2014, 837891. [CrossRef]

12. Britton, G.; Liaaen-Jensen, S.; Pfander, H. Carotenoids—Handbook; Birkhäuser Verlag: Basel, Switzerland, 2004; pp. 1-625.

13. Soliev, A.B.; Enomoto, K. Antitumor Pigments from Marine Bacteria. In Marine Biomaterials: Characterization, Isolation and Applications; Kim, S., Ed.; CRC Press: London, UK, 2013; pp. 149-171.

14. Williamson, N.R.; Fineran, P.C.; Gristwood, T.; Chawrai, S.R.; Leeper, F.J.; Salmond, G.P.C. Anticancer and immunosuppressive properties of bacterial prodiginines. Future Microbiol. 2007, 2, 605-618. [CrossRef]

15. Gürses, A.; Açıkyıldız, M.; Güneş, K.; Gürses, M.S. Dyes and Pigments; Springer: Cham, Switzerland, 2016; pp. 1-83.

16. Delgado-Vargas, F.; Paredes-López, O. Natural Colorants for Food and Nutraceutical Uses; CRC Press LLC: Boca Raton, FL, USA, 2003; pp. 1-344.

17. Babitha, S. Microbial Pigments. In Biotechnology for Agro-Industrial Residues Utilisation; Nigam, P.S., Pandey, A., Eds.; Springer: Berlin/Heidelberg, Germany, 2009; pp. 147-162.

18. Caro, Y.; Anamale, L.; Fouillaud, M.; Laurent, P.; Petit, T.; Dufosse, L. Natural hydroxyanthraquinoid pigmentsas potent food grade colorants: An overview. Nat. Prod. Bioprospect. 2012, 2, 174-193. [CrossRef]

19. Misawa, N. Carotenoid $\beta$-ring hydroxylase and ketolase from marine bacteria-promiscuous enzymes for synthesizing functional xanthophylls. Mar. Drugs 2011, 9, 757-771. [CrossRef] [PubMed]

20. Heider, S.A.; Peters-Wendisch, P.; Wendisch, V.F.; Beekwilder, J.; Brautaset, T. Metabolic engineering for the microbial production of carotenoids and related products with a focus on the rare $\mathrm{C}_{50}$ carotenoids. Appl. Microbiol. Biotechnol. 2014, 98, 4355-4368. [CrossRef] [PubMed]

21. Dufossé, L. Current and Potential Natural Pigments from Microorganisms (Bacteria, Yeasts, Fungi, Microalgae). In Handbook on Natural Pigments in Food and Beverages; Carle, R., Schweiggert, R.M., Eds.; Elsevier Ltd.: Cambridge, UK, 2016; pp. 337-354.

22. Revuelta, J.L.; Ledesma-Amaro, R.; Jiménez, A. Industrial Production of Vitamin B2 by Microbial Fermentation. In Industrial Biotechnology of Vitamins, Biopigments, and Antioxidants, 1st ed.; Vandamme, E.J., Revuelta, J.L., Eds.; Wiley-VCH Verlag GmbH \& Co. KGaA: Weinheim, Germany, 2016; pp. 17-40.

23. Baxter, B.K.; Gunde-Cimerman, N.; Oren, A. Salty sisters: The women of halophiles. Front. Microbiol. 2014, 5, 192. [CrossRef] [PubMed]

24. Nelis, H.J.; de Leenheer, A.P. Microbial production of carotenoids other than $\beta$-carotene. In Biotechnology of Vitamins, Pigments and Growth Factors; Vandamme, J., Ed.; Elsevier: Essex, UK, 1989; pp. 43-80.

25. Joshi, V.K.; Attri, D.; Bala, A.; Bhushan, S. Microbial pigments. Indian J. Biotechnol. 2003, 2, 362-369.

26. Feng, Y.; Shao, Y.; Zhou, Y.; Chen, W.; Chen, F. Monascus Pigments. In Industrial Biotechnology of Vitamins, Biopigments, and Antioxidants, 1st ed.; Vandamme, E.J., Revuelta, J.L., Eds.; Wiley-VCH Verlag GmbH \& Co. KGaA: Weinheim, Germany, 2016; pp. 497-535.

27. Hodgkiss, W.; Liston, M.; Godwin, T.W.; Jamikorn, M. The isolation and description of two marine micro-organisms with special reference to their pigment production. J. Gen. Microbiol. 1954, 11, 488-4150. [CrossRef] [PubMed] 
28. Giddings, L.; Newman, D.J. Bioactive Compounds from Terrestrial Extremophiles; Springer: Cham, Switzerland, 2015; pp. 1-75.

29. Okazaki, T.; Kitahara, T.; Okami, Y. Studies on marine microorganisms. IV. A new antibiotic SS-228 Y produced by Chainia isolated from shallow sea mud. J. Antibiot. 1975, 28, 176-184. [CrossRef]

30. Franks, A.; Haywood, P.; Holmstöm, C.; Egan, S.; Kjelleberg, S.; Kumar, N. Isolation and structure elucidation of a novel yellow pigment from the marine bacterium Pseudoalteromonas tunicata. Molecules 2005, 10, 1286-1291. [CrossRef]

31. Blackman, A.; Li, C. New tambjamine alkaloids from the marine bryozoan Bugula dentata. Aust. J. Chem. 1994, 47, 1625-1629. [CrossRef]

32. Pinkerton, D.M.; Banwell, M.G.; Garson, M.J.; Kumar, N.; de Moraes, M.O.; Cavalcanti, B.C.; Barros, F.W.; Pessoa, C. Antimicrobial and cytotoxic activities of synthetically derived tambjamines C and E-J, BE-18591, and a related alkaloid from the marine bacterium Pseudoalteromonas tunicata. Chem. Biodivers. 2010, 7, 1311-1324. [CrossRef]

33. Hermansson, M.; Jones, G.W.; Kjelleberg, S. Frequency of antibiotic and heavy metal resis-tance, pigmentation, and plasmids in bacteria of the marine airwater interface. Appl. Environ. Microbiol. 1987, 53, 2338-2342. [PubMed]

34. Miteva, V.I.; Sheridan, P.P.; Brenchley, J.E. Phylogenetic and physiological diversity of micro-organisms isolated from a deep greenland glacier ice core. Appl. Environ. Microbiol. 2004, 70, 202-213. [CrossRef] [PubMed]

35. Zhang, X.F.; Yao, T.D.; Tian, L.D.; Xu, S.J.; An, L.Z. Phylogenetic and physiological diversity of bacteria isolated from puruogangri ice core. Microb. Ecol. 2008, 55, 476-488. [CrossRef]

36. Agogué, H.; Joux, F.; Obernosterer, I.; Lebaron, P. Resistance of marine bacterioneuston to solar radiation. Appl. Environ. Microbiol. 2005, 71, 5282-5289. [CrossRef] [PubMed]

37. Khanafari, A.; Khavarinejad, D.; Mashinchian, A. Solar salt lake as natural environmental source for extraction halophilic pigments. Iran. J. Microbiol. 2009, 2, 103-109.

38. Yurkov, V.V.; Krieger, S.; Stackebrandt, E.; Beatty, J.T. Citromicrobium bathyomarinum, a novel aerobic bacterium isolated from deep-sea hydrothermal vent plume waters that contains photosynthetic pigment-protein complexes. J. Bacteriol. 1999, 181, 4517-4525. [PubMed]

39. Hathaway, J.J.M.; Garcia, M.G.; Balasch, M.M.; Spilde, M.N.; Stone, F.D.; Dapkevicius, M.D.L.N.; Amorim, I.R.; Gabriel, R.; Borges, P.A.; Northup, D.E. Comparison of bacterial diversity in Azorean and Hawaiian lava cave microbial mats. Geomicrobiol. J. 2014, 31, 205-220. [CrossRef]

40. Ramesh, C.H.; Mohanraju, R.; Narayana, S.; Murthy, K.N.; Karthick, P. Molecular characterization of marine pigmented bacteria showing antibacterial activity. Indian J. Mar. Sci. 2017, 46, 2081-2087.

41. Nedashkovskaya, O.I.; Ludwig, W. Family II. Cyclobacteriaceae fam. nov. In Bergey's Manual of Systematic Bacteriology, 2nd ed.; Krieg, N.R., Ludwig, W., Whitman, W., Hedlund, B.P., Paster, B.J., Staley, J.T., Ward, N., Brown, D., Parte, A., Eds.; Springer: New York, NY, USA, 2011; Volume 4, pp. 423-444.

42. Méndez-Zavala, A.; Contreras-Esquivel, J.C.; Lara-Victoriano, F.; Rodríguez-Herrera, R.; Aguilar, C.N. Fungal production of the red pigment using a xerophilic strain Penicillium purpurogenum GH-2. Revista Mexicana de Ingeniería Química 2007, 6, 267-273.

43. Van den Hoek, C.; Mann, D.G.; Jahns, H.M. Algae: An Introduction to Phycology; Cambridge University Press: Cambridge, UK, 1995; 637p.

44. Lee, R.E. Phycology; Cambridge University Press: Cambridge, UK, 1999; pp. 1-624.

45. Graham, L.; Wilcox, L. Algae; Prentice-Hall: Englewood Cliffs, NJ, USA, 2000; 700p.

46. Likens, G.E. Plankton of Inland Waters: A Derivative of Encyclopedia of Inland Waters, 1st ed.; Academic Press/Elsevier: New York, NY, USA, 2010; 412p.

47. Takaichi, S. Carotenoids in Algae: Distributions, Biosyntheses and Functions. Mar. Drugs 2011, 9, 1101-1118. [CrossRef]

48. Roy, S.; Llewellyn, C.; Egeland, E.S.; Johnsen, G. Phytoplankton Pigments: Characterization, Chemotaxonomy and Applications in Oceanography; Cambridge University Press: Cambridge, UK, 2011; 845p.

49. Stafsnes, M.H.; Bruheim, P. Pigmented Marine Heterotrophic Bacteria. In Marine Biomaterials: Characterization, Isolation and Applications; Kim, S., Ed.; CRC Press, Taylor \& Francis Group: London, UK, 2013; pp. 117-148.

50. Dieser, M.; Greenwood, M.; Foreman, C.M. Carotenoid pigmentation in Antarctic hetero-trophic bacteria as a strategy to withstand environmental stresses. Arct. Antarct. Alp. Res. 2010, 42, 396-405. [CrossRef] 
51. Egan, S.; James, S.; Holmström, C.; Kjelleberg, S. Correlation between pigmentation and antifouling compounds produced by Pseudoalteromonas tunicata. Environ. Microbiol. 2002, 4, 433-442. [CrossRef] [PubMed]

52. Mandelli, F.; Miranda, V.; Rodrigues, E.; Mercadante, A. Identification of carotenoids with high antioxidant capacity produced by extremophile microorganisms. World J. Microbiol. Biotechnol. 2012, 28, 1781-1790. [CrossRef] [PubMed]

53. Chew, B.P.; Park, J.S. Carotenoid action on the immune response. J. Nutr. 2004, 134, 257S-261S. [CrossRef] [PubMed]

54. Konzen, M.; Marco, D.D.; Cordova, C.A.S.; Vieira, T.O.; Antônio, R.V.; Creczynski-Pasa, T.B. Antioxidant properties of violacein: Possible relation on its biological function. Bioorg. Med. Chem. 2006, 14, 8307-8313. [CrossRef] [PubMed]

55. Pierson, L.; Pierson, E. Metabolism and function of phenazines in bacteria: Impacts on the behavior of bacteria in the environment and biotechnological processes. Appl. Microbiol. Biotechnol. 2010, 86, 1659-1670. [CrossRef] [PubMed]

56. Albuquerque, L.; Costa, M.S. The Family Thermaceae. In The Prokaryotes-Other Major Lineages of Bacteria and the Archaea; Rosenberg, E., DeLong, E.F., Lory, S., Stackebrandt, E., Thompson, F., Eds.; Springer: Berlin/Heidelberg, Germany, 2014; pp. 955-987.

57. Boric, M.; Danevcic, T.; Stopar, D. Prodigiosin from Vibrio sp. DSM 14379: A new UV-protective pigment. Microb. Ecol. 2011, 62, 528-536. [CrossRef]

58. Plonka, P.M.; Grabacka, M. Melanin synthesis in microorganisms-Biotechnological and medical aspects. Acta Biochim. Pol. 2006, 53, 429-443.

59. Coyne, V.E.; Al-Harthi, L. Induction of melanin biosynthesis in Vibrio cholerae. Appl. Environ. Microbiol. 1992, 58, 2861-2865.

60. Matz, C.; Deines, P.; Boenigt, J.; Arndt, H.; Eberl, L.; Kjelleberg, S.; Jürgens, K. Impact of violacein-producing bacteria on survival and feeding of bacterivorous nanoflagellates. Appl. Environ. Microbiol. 2004, 70, 1593-1599. [CrossRef]

61. Visca, P.; Imperi, F.; Lamont, I.L. Pyoverdine siderophores: From biogenesis to biosignificance. Trends Microbiol. 2006, 15, 22-30. [CrossRef] [PubMed]

62. Nair, S.; Chandramohan, D.; Bharathi, P.A.L. Differential sensitivity of pigmented and non-pigmented marine bacteria to metals and antibiotics. Water Res. 1992, 26, 431-434. [CrossRef]

63. Margalith, P.Z. Pigment Microbiology; Chapman \& Hall: London, UK, 1992; pp. 1-156.

64. Gessler, N.N.; Egorova, A.S.; Belozerskaya, T.A. Fungal anthraquinones. Appl. Biochem. Microbiol. 2013, 49, 85-99. [CrossRef]

65. Soni, S.K. Microbes: A Source of Energy for the 21st Century; New India Publishing Agency: New Delhi, India, 2007; pp. 1-590.

66. Tong, Y.Y.; Lighthart, B. Solar radiation is shown to select for pigmented bacteria in the ambient outdoor atmosphere. Photochem. Photobiol. 1997, 65, 103-106. [CrossRef]

67. Stafsnes, M.; Josefsen, K.; Kildahl-Andersen, G.; Valla, S.; Ellingsen, T.; Bruheim, P. Isolation and characterization of marine pigmented bacteria from Norwegian coastal waters and screening for carotenoids with UVA-blue light absorbing properties. J. Microbiol. 2010, 48, 16-23. [CrossRef] [PubMed]

68. Nakamura, Y.; Asada, C.; Sawada, T. Production of antibacterial violet pigment by psychrotropic bacterium RT102 strain. Biotechnol. Bioprocess Eng. 2003, 8, 37-40. [CrossRef]

69. Liu, G.Y.; Nizet, V. Color me bad: Microbial pigments as virulence factors. Trends Microbiol. 2009, 17, 406-413. [CrossRef] [PubMed]

70. Oren, A. A short history of the symposia on halophilic microorganisms: From Rehovot 1978 to Beijing 2010. In Halophiles and Hypersaline Environments: Current Research and Future Trends; Ventosa, A., Oren, A., Ma, Y., Eds.; Springer: Berlin, Germany, 2011; pp. 373-382.

71. Lev-Yadun, S.; Halpern, M. Ergot (Claviceps purpurea)—An aposematic fungus. Symbiosis 2007, 43, 105-108.

72. Venil, C.K.; Aruldass, C.A.; Dufossé, L.; Zakaria, Z.A.; Ahmad, W.A. Current perspective on bacterial pigments: Emergingsustainable compounds with coloring and biological properties for the industry-An incisive evaluation. RSC Adv. 2014, 4, 39523-39529. [CrossRef]

73. Nigam, P.S.; Luke, J.S. Food additives: Production of microbial pigments and their antioxidant properties. Curr. Opin. Food Sci. 2016, 7, 93-100. [CrossRef] 
74. Capelli, G.C.; Cysewski, G. The Worlds' Best Kept Health Secret Natural Astaxanthin; Cyanotech Corporation: Kailua-Kona, HI, USA, 2013; pp. 1-202.

75. Ananya, A.K.; Ahmad, I.Z. Cyanobacteria "the blue green algae" and its novel applications: A brief review. Int. J. Innov. Appl. Stud. 2014, 7, 251-261.

76. Rao, M. Microbes and Non-Flowering Plants: Impact and Applications; Ane Books Pvt Ltd.: New Delhi, India, 2009; 565p.

77. Sonani, R.R.; Rastogi, R.P.; Patel, R.; Madamwar, D. Recent advances in production, purification and applications of phycobiliproteins. World J. Biol. Chem. 2016, 26, 100-109. [CrossRef] [PubMed]

78. Konuray, G.; Erginkaya, Z. Antimicrobial and antioxidant properties of pigments synthesized from microorganisms. In The Battle against Microbial Pathogens: Basic Science, Technological Advances and Educational Programs; Méndez-Vilas, A., Ed.; Formatex Research Center: Badajoz, Spain, 2015; pp. 27-33.

79. Begum, H.; Yusoff, F.M.; Banerjee, S.; Khatoon, H.; Shariff, M. Availability and utilization of pigments from microalgae. Crit. Rev. Food Sci. Nutr. 2015, 56, 2209-2222. [CrossRef] [PubMed]

80. Stankovic, N.; Senerovic, L.; Ilic-Tomic, T.; Vasiljevic, B.; Nikodinovic-Runic, J. Properties and applications of undecylprodigiosin and other bacterial prodigiosins. Appl. Microbiol. Biotechnol. 2014, 98, 3841-3858. [CrossRef] [PubMed]

81. Durán, N.; Justo, G.Z.; Ferreira, C.V.; Melo, P.S.; Cordi, L.; Martins, D. Violacein: Properties and biological activities. Biotechnol. Appl. Biochem. 2007, 48, 127-133. [PubMed]

82. Andersen, R.J.; Wolfe, M.S.; Faulkner, D.J. Autotoxic antibiotic production by a marine Chromobacterium. Mar. Biol. 1974, 27, 281-285. [CrossRef]

83. Schneemann, I.; Wiese, J.; Kunz, A.L.; Imhoff, J.F. Genetic approach for the fast discovery of phenazine producing bacteria. Mar. Drugs 2011, 9, 772-789. [CrossRef]

84. Shirata, A.; Tsukamoto, T.; Yasui, H.; Kato, H.; Hayasaka, S.; Kojima, A. Production of bluish-purple pigments by Janthinobacterium lividum isolated from the raw silk and dyeing with them. Nippon Sanshigaku Zasshi 1997, $66,377-385$.

85. Koyama, J. Anti-infective quinone derivatives of recent patents. Recent Pat. Anti-Infect. Drug Discov. 2006, 1, 113-125. [CrossRef]

86. Zhang, J.; Shen, Y.; Liu, J.; Wei, D. Antimetastatic effect of prodigiosin through inhibition of tumor invasion. Biochem. Pharmacol. 2005, 69, 407-414. [CrossRef]

87. Kawauchi, K.; Shibutani, K.; Yagisawa, H.; Kamata, H.; Nakatsuji, S.I.; Anzai, H.; Yokoyama, Y.; Ikegami, Y.; Moriyama, Y.; Hirata, H. A possible immunosuppressant, cycloprodigiosin hydrochloride, obtained from Pseudoalteromonas denitrificans. Biochem. Biophys. Res. Commun. 1997, 237, 543-547. [CrossRef] [PubMed]

88. Han, S.B.; Kim, H.M.; Kim, Y.H.; Lee, C.W.; Jang, E.S.; Son, K.H.; Sung, U.K.; Kim, Y.K. T-cell specific immunosuppression by prodigiosin isolated from Serratia marcescens. Int. J. Immunopharmacol. 1998, 20, 1-13. [CrossRef]

89. Songia, S.; Mortellaro, A.; Taverna, S.; Fornasiero, C.; Scheiber, E.A.; Erba, E.; Colotta, F.; Mantovani, A.; Isetta, A.M.; Golay, J. Characterization of the new immunosuppressive drug undecylprodigiosin in human lymphocytes: Retinoblastoma protein, cyclin-dependent kinase-2, and cyclin-dependent kinase-4 as molecular targets. J. Immunol. 1997, 158, 3987-3995. [PubMed]

90. Fouillaud, M.; Venkatachalam, M.; Girard-Valenciennes, E.; Caro, Y.; Dufossé, L. Anthraquinones and derivatives from marine-derived fungi: Structural diversity and selected biological activities. Mar. Drugs 2016, 14, 64. [CrossRef] [PubMed]

91. Lee, C.L.; Hung, H.K.; Wang, J.J.; Pan, T.M. Red mold dioscorea has greater hypolipidemic and antiatherosclerotic effect than tradi-tional redmold rice andunfermented dioscorea in hamsters. J. Agric. Food Chem. 2007, 55, 7162-7169. [CrossRef] [PubMed]

92. Wu, C.L.; Lee, C.L.; Pan, T.M. Red mold dioscorea has a greater antihypertensive effect than traditional red mold rice in spontaneously hypertensive rats. J. Agric. Food Chem. 2009, 57, 5035-5041. [CrossRef]

93. Fox, R.D. Spirulina, the alga that can end malnutrition. Futurist 1985, 19, 30-35.

94. Montaner, B.; Pérez-Tomás, R. Prodigiosin-induced apoptosis in human colon cancer cells. Life Sci. 2001, 68, 2025-2036. [CrossRef]

95. Zheng, L.; Yan, X.; Han, X.; Chen, H.; Lin, W.; Lee, F.S.; Wang, X. Identification of norharman as the cytotoxic compound pro-duced by the sponge (Hymeniacidon perleve) associated marine bacterium Pseudoalteromonas piscicida and its apoptotic effect on cancer cells. Biotechnol. Appl. Biochem. 2006, 44, 135-142. 
96. Chincholkar, S.; Thomashow, L. Microbial Phenazines: Biosynthesis, Agriculture and Health; Springer: Berlin/Heidelberg, Germany, 2013; pp. 1-243.

97. Mojib, N.; Philpott, R.; Huang, J.P.; Niederweis, M.; Bej, A.K. Antimycobacterial activity of in vitro of pigments isolated from Antartic bacteria. Antonie van Leeuwenhoek 2010, 98, 531-540. [CrossRef]

98. Holmström, C.; Egan, S.; Franks, A.; McCloy, S.; Kjelleberg, S. Antifouling activities expressed by marine surface associated Pseudoalteromonas species. FEMS Microbiol. Ecol. 2002, 41, 47-58. [CrossRef]

99. Priya, K.A.; Satheesh, S.; Ashokkumar, B.; Varalakshmi, P.; Selvakumar, G.; Sivakumar, N. Antifouling activity of prodigiosin from estuarine isolate of Serratia marcescens CMST 07. In Microbiological Research in Agroecosystem Management; Velu, R.K., Ed.; Springer: New Delhi, India, 2013; Volume XVI, pp. 11-21.

100. Jeong, H.; Yim, J.H.; Lee, C.; Choi, S.H.; Park, Y.K.; Yoon, S.H.; Hur, C.G.; Kang, H.Y.; Kim, D.; Lee, H.H.; et al. Genomic blueprint of Hahella chejuensis, a marine microbe producing an algicidal agent. Nucleic Acids Res. 2005, 33, 7066-7073. [CrossRef] [PubMed]

101. Kim, D.; Kim, J.; Yim, J.H.; Kwon, S.K.; Lee, C.H.; Lee, H.K. Red to red-The marine bacterium Hahella chejuensis and its product prodigiosin for mitigation of harmful algal blooms. J. Microbiol. Biotechnol. 2008, 18, 1621-1629. [PubMed]

102. Sakaki, T.; Shibata, M.; Mukai, K.; Sakai, M.; Wakamatsu, K.; Miyauchi, S. Chlorociboria aeruginosa pigment as algicide. Japanese Kokai Tokkyo Koho 2002, 2002, 2002291493.

103. Rai, M. Advances in Fungal Biotechnology; I. K. International Pvt Ltd.: New Delhi, India, 2009; p. 545.

104. Quereshi, S.; Khan, A.A.; Pandey; Khim, A.K. Anthraquinone pigment with herbicidal potential from Phoma herbarum FGCC\#54. Chem. Nat. Compd. 2011, 47, 521.

105. Haun, M.; Pereira, M.F.; Hoffman, M.E.; Joyas, A.; Campos, V.; Filardi, L.D.; De Castro, S.L.; Duran, N. Bacterial chemistry. VI. Biological activities and cytotoxicity of 1,3-dihydro-2H-indol-2-one derivatives. Biol. Res. 1992, 25, 21-25. [PubMed]

106. Lopes, S.C.P.; Blanco, Y.C.; Justo, G.Z.; Nogueira, P.A.; Rodrigues, F.L.S.; Goelnitz, U.; Wunderlich, G.; Facchini, G.; Brocchi, M.; Duran, N.; et al. Violacein extracted from Chromobacterium violaceum inhibits Plasmodium growth in vitro and in vivo. Antimicrob. Agents Chemother. 2009, 53, 2149-2152. [CrossRef]

107. Williams, R.P.; Quadri, S.M. The pigments of Serratia. In The genus Serratia; Graevenitz, A.V., Rubin, S.J., Eds.; CRC Press Inc.: Boca Raton, FL, USA, 1980; pp. 31-75.

108. Leon, L.L.; Miranda, C.C.; De Souza, A.O.; Duran, N. Antileishmanial activity of the violacein extracted from Chromobacterium violaceum. J. Antimicrob. Chemother. 2001, 3, 449-450. [CrossRef]

109. Moraes, C.S.; Seabra, S.H.; Castro, D.P.; Brazil, R.P.; de Souza, W.; Garcia, E.S.; Azambuja, P. Leishmania chagasi interactions with Serratia marcescens: Ultrastructural studies, lysis and carbohydrate effects. Exp. Parasitol. 2008, 118, 561-568. [CrossRef]

110. Durản, N.; Justo, G.Z.; Melo, P.S.; DeAzevedo, M.B.M.; Brito, A.R.M.S.; Almeida, A.B.; Haun, M. Evaluation of the antiulcerogenic activity of violacein and its modulation by the inclusion complexation with beta-cyclodextrin. Can. J. Physiol. Pharmacol. 2003, 81, 387-396. [CrossRef]

111. Duran, N.; Melo, P.S.; Haun, M. In Vitro evaluation of violacein on AIDS-related lumphoma and human tumor cell lines. In Proceedings of the 25th Annual Meetings of the Brazilian Society of Biochemistry and Molecular Biology, Sociedade Brasi-leira de Bioquimica e Biologia Molecular (SBBq), Caxambu, Brazil, 1996; Available online: https://scholar.google.co.in/scholar?hl=en\&as_sdt=0\%2C5\&q=In+Vitro+evaluation+of+ violacein+on+AIDS-related+lumphoma+and+human+tumor+cell+lines\&btnG= (accessed on 31 December 2016).

112. Kim, H.S.; Hayashi, M.; Shibata, Y.; Wataya, Y.; Mitamura, T.; Horii, T.; Kawauchi, K.; Hirata, H.; Tsuboi, S.; Moriyama, Y. Cycloprodigiosin hydrochloride obtained from Pseudoalteromonas denitrificansis a potent antimalarial agent. Biol. Pharm. Bull. 1999, 22, 532-534. [CrossRef] [PubMed]

113. Lazaro, J.E.; Nitcheu, J.; Predicala, R.Z.; Mangalindan, G.C.; Nesslany, F.; Marzin, D.; Concepcion, G.P.; Diquet, B. Heptyl prodigiosin, a bacterial metabolite, is anti-malarial in vivo and nonmutagenic in vitro. J. Nat. Toxins 2002, 11, 367-377. [PubMed]

114. Liu, Y.T.; Sui, M.J.; Ji, D.D.; Wu, I.H.; Chou, C.; Chen, C.C. Protection from UV irradiation by melanin of mosquitocidal activity of Bt.var. israeliensis. J. Invertebr. Pathol. 1993, 62, 131-136. [CrossRef] [PubMed]

115. Genes, C.; Baquero, E.; Echeverri, F.; Maya, J.D.; Triana, O. Mitochondrial dysfunction in Trypanosoma cruzi: The role of Serratia marcescens prodigiosin in the alternative treatment of Chagas disease. Parasites Vectors 2011, 4, 66. [CrossRef] [PubMed] 
116. Azambuja, P.; Feder, D.; Garcia, E.S. Isolation of Serratia marcescens in the midgut of Rhodnius prolixus: Impact on the establishment of the parasite Trypanosoma cruzi in the vector. Exp. Parasitol. 2004, 107, 89-96. [CrossRef] [PubMed]

117. Sankari, M.K.; Jonathan, E.I.; Ardhanareeswaran, N. Isolation of phenazine and its activity against root-knot nematode, Meloidogyne incognita. Indian J. Biotehnol. 2014, 43, 180-183.

118. Shi, Y.C.; Liao, J.W.; Pan, T.M. Antihypertriglyceridemia and anti-inflammatory activities of Monascus-fermented dioscorea in streptozotocin-induced diabetic rats. Exp. Diabetes Res. 2011, 2011, 710635. [CrossRef] [PubMed]

119. Lee, B.H.; Hsu, W.H.; Liao, T.H.; Pan, T.M. The Monascus metabolite monascin against TNF- $\alpha$-induced insulin resistance via suppressing PPAR- $\gamma$ phos-phorylation in C2C12 myotubes. Food Chem. Toxicol. 2011, 49, 2609-2617. [CrossRef]

120. Lee, C.; Hung, Y.; Hsu, Y.; Pan, T. Monascin and Ankaflavin have more anti-atherosclerosis effect and less side effect involving increasing creatinine phosphokinase activity than Monacolin $\mathrm{K}$ under the same dosages. J. Agric. Food Chem. 2013, 61, 143-150. [CrossRef]

121. Kishimoto, Y.; Yoshida, H.; Kondo, K. Potential Anti-Atherosclerotic Properties of Astaxanthin. Mar. Drugs 2016, 14, 35. [CrossRef]

122. Osawa, A.; Ishii, Y.; Sasamura, N.; Morita, M.; Kasai, H.; Maoka, T.; Shindo, K. Characterization and antioxidative activities of rare $\mathrm{C}_{50}$ carotenoids-sarcinaxanthin, sarcinaxanthin monoglucoside, and sarcinaxanthin diglucoside obtained from Micrococcus yunnanensis. J. Oleo Sci. 2010, 59, 653-659. [CrossRef] [PubMed]

123. Martin, H.; Kock, S.; Scherrers, R.; Lutter, K.; Wagener, T.; Hundsdörfer, C.; Frixel, S.; Schaper, K.; Ernst, H.; Schrader, W.; et al. 3,3'-Dihydroxyisorenieratene, a Natural Carotenoid with Superior Antioxidant and Photoprotective Properties. Angew. Chem. 2009, 48, 400-403. [CrossRef] [PubMed]

124. Teo, I.T.; Chui, C.H.; Tang, J.C.O.; Lau, F.Y.; Cheng, G.Y.M.; Wong, R. Antiproliferation and induction of cell death of Phaffia rhodozyma (Xanthophyllomyces dendrorhous) extract fermented by brewer malt waste on breast cancer cells. Int. J. Mol. Med. 2005, 16, 931-936. [CrossRef] [PubMed]

125. Lee, H.; Kim, Y.; Kim, Y. The effect of beta-carotene on neuroblastoma stemness. FASEB J. 2012, 26. [CrossRef]

126. Kim, J.H.; Kim, Y.O.; Jeun, J.; Choi, D.Y.; Shin, C.S. L-Trp and L-Leu-OEt derivatives of the Monascus pigment exert high anti-obesity effects on mice. Biosci. Biotechnol. Biochem. 2010, 74, 304-308. [CrossRef] [PubMed]

127. Jou, P.C.; Ho, B.Y.; Hsu, Y.W.; Pan, T.M. The effect of Monascus secondary polyketide metabolites, monascin and ankaflavin, on adipogenesis and lipolysis activity in 3T3-L1. J. Agric. Food Chem. 2010, 58, 12703-12709. [CrossRef]

128. Choe, D.; Lee, J.; Woo, S.; Shin, C.S. Evaluation of the amine derivatives of Monascus pigment with anti-obesity activities. Food Chem. 2012, 134, 315-323. [CrossRef]

129. Wang, Z.; Lin, B.; Mostaghim, A.; Rubin, R.A.; Glaser, E.R.; Mittraparp-arthorn, P.; Thompson, J.R.; Vuddhakul, V.; Vora, G.J. Vibrio campbellii hmgA-mediated pyomelanization impairs quorum sensing, virulence, and cellular fitness. Front. Microbiol. 2013, 4, 379. [CrossRef]

130. Srianta, I.; Kusumawati, N.; Nugerahani, I.; Artanti, N.; Xu, G.R. In vitro $\alpha$-glucosidase inhibitory activity of Monascus-fermented durian seed extracts. Int. Food Res. J. 2013, 20, 533-536.

131. Lung, T.; Liao, L.; Wang, J.; Wei, B.; Huang, P.; Lee, C. Metals of deep ocean water increase the anti-adipogenesis effect of monascus-fermented product via modulating the monascin and ankaflavin production. Mar. Drugs 2016, 14, 106. [CrossRef]

132. Lindquist, N.; Fenical, W. New tambjamine class alkaloids from the marine ascidian Atapozoa sp. and its nudibranch predators-Origins of the tambjamines in atapozoa. Experientia 1991, 47, 504-506. [CrossRef]

133. Chakdar, H.; Pabbi, S. Extraction and purification of Phycoerythrin from Anabaena variabilis (CCC421). Phykos 2012, 42, 25-31.

134. Speitling, M.; Smetanina, O.F.; Kuznetsova, O.F.; Laatsch, H. Bromoalterochromides A and A', unprecedented chromopeptides from a marine Pseudoalteromonas maricaloris strain KMM 636T. J. Antibiot. 2007, 60, 36-42. [CrossRef] [PubMed]

135. Grossart, H.; Thorwest, M.; Plitzko, I.; Brinkhoff, T.; Simon, M.; Zeeck, A. Production of a blue pigment (Glaukothalin) by marine Rheinheimera spp. Int. J. Microbiol. 2009, 2009, 701735. [CrossRef] [PubMed] 
136. Tebben, J.; Tapiolas, D.M.; Motti, C.A.; Abrego, D.; Negri, A.P.; Blackall, L.L.; Steinberg, P.D.; Harder, T. Induction of larval metamorphosis of the coral Acropora millepora by tetrabromopyrrole isolated from a Pseudoalteromonas bacterium. PLoS ONE 2011, 6, e19082. [CrossRef]

137. George, S.B.; Lawrence, J.M.; Lawrence, A.L.; Smiley, J.; Plank, L. Carotenoids in the adult diet enhance egg and juvenile production in the sea urchin Lytechinus variegatus. Aquaculture 2001, 199, 353-369. [CrossRef]

138. Meena, K.K.; Kumar, M.; Kalyuzhnaya, M.G.; Yandigen, M.S.; Singh, D.P.; Saxena, A.K.; Arora, D. Epiphytic pink-pigmentedmethylotrophic bacteria enhance germination and seedling growth of wheat (Triticum aestivum) by producing phytohormone. Antonie Van Leeuwenhoek 2012, 101, 777-786. [CrossRef]

139. Martínkovả, L. Biological activities of oligoketide pigments of Monascus purpureus. Food Addit. Contam. 1999, 16, 15-24. [CrossRef]

140. Buck, J.D. Effects of medium composition on the recovery of bacteria from sea water. J. Exp. Mar. Biol. Ecol. 1974, 15, 25-34. [CrossRef]

141. Reichenbach, H.; Kleinig, H.; Achenbach, H. The pigments of Flexibacter elegans: Novel and chemosystematically useful compounds. Arch. Microbiol. 1974, 101, 131-144. [CrossRef]

142. Hajjaj, H.; Blanc, P.; Groussac, E.; Uribelarrea, J.L.; Goma, G.; Loubiere, P. Kinetic analysis of red pigment and citrinin by Monascus rubber as a function of organic acid accumulation. Enzym. Microb. Technol. 2000, 27, 619-625. [CrossRef]

143. De Carvalho, J.C. Microbial Pigments. In Biotransformation of Waste Biomass into High Value Biochemicals; Brar, S.K., Dhillon, G.S., Soccol, C.R., Eds.; Springer: New York, NY, USA, 2014; pp. 73-98.

144. Ghosh, A.; Goyal, A.; Jain, R.K. Study of methanol-induced phenotypic changes in a novel strain of Acinetobacter lwoffii. Arch. Microbiol. 2007, 188, 533-539. [CrossRef] [PubMed]

145. Saviola, B. Pigments and Pathogenesis. J. Mycobact. Dis. 2014, 4. [CrossRef]

146. Robledo, J.A.; Murillo, A.M.; Rouzaud, F. Physiological Role and Potential Clinical Interest of Mycobacterial Pigments. IUBMB Life 2011, 63, 71-78. [CrossRef] [PubMed]

147. Mulders, K.J.M. Phototrophic pigment production with microalgae: Biological constraints and opportunities. J. Phycol. 2014, 50, 229-242. [CrossRef] [PubMed]

148. Yamashita, M.; Nakagawa, Y.; Li, H.; Matsuyama, T. Silica gel-dependent production of prodigiosin and serrawettins by Serratia marcescens in a liquid culture. Microbes Environ. 2001, 16, 250-254. [CrossRef]

149. Chen, W.C.; Yu, W.J.; Chang, C.C.; Chang, J.S.; Huang, S.H.; Chang, C.H.; Chen, S.Y.; Chien, C.C.; Yao, C.L.; Chen, W.M.; et al. Enhancing production of prodigiosin from Serratia marcescens $\mathrm{C} 3$ by statistical experimental design and porous carrier addition strategy. Biochem. Eng. J. 2013, 78, 93-100. [CrossRef]

150. Guedes, A.C.; Amaro, H.M.; Malcata, F.X. Microalgae as Sources of Carotenoids. Mar. Drugs 2011, 9, 625-644. [CrossRef]

151. Velmurugan, P.; Kamala-Kannan, S.; Balachandar, V.; Lakshmanaperumalsamy, P.; Chae, J.; Oh, B. Natural pigment extraction from five filamentous fungi for industrial applications and dyeing of leather. Carbohydr. Polym. 2010, 79, 262-268. [CrossRef]

152. Yurkova, A.M.; Vustin, M.M.; Tyaglov, B.V.; Maksimova, I.A.; Sineokiy, S.P. Pigmented basidiomycetous yeasts are a promising source of carotenoids and ubiquinone Q10. Microbiology 2008, 77, 1-6. [CrossRef]

153. Ahmad, W.A.; Venil, C.K.; Aruldass, C.A. Production of Violacein by Chromobacterium violaceum Grown in Liquid Pineapple Waste: Current Scenario. In Beneficial Microorganisms in Agriculture, Aquaculture and Other Areas, Microbiology Monographs; Liong, M.T., Ed.; Springer: Berlin/Heidelberg, Germany, 2015; Volume 29, pp. 45-58.

154. Carvalho, J.C.; Bicas, J.L.; Fernández, D.E.R.; Woiciechowski, A.L.; Medeiros, A.B.P.; Soccol, C.R. Natural Colorants from Microorganisms. In Biotechnological Production of Natural Ingredients for Food Industry, 1st ed.; Bicas, J.L., Maróstica Jr, M.R., Pastore, G.M., Eds.; Bentham Science Publishers: Sharjah, UAE, 2016; pp. 288-321.

155. Taskin, M.; Sisman, T.; Erdal, S.; Kurbanoglu, E.B. Use of waste chicken feathers as peptone for production of carotenoids in submerged culture of Rhodotorula glutinis MT-5. Eur. Food Res. Technol. 2011, 233, 657-665. [CrossRef]

156. Wang, Y.; Nakajima, A.; Hosokawa, K.; Soliev, A.B.; Osaka, I.; Arakawa, R.; Enomoto, K. Cytotoxic prodigiosin family pigments from Pseudoalteromonas sp.1020R isolated from the Pacific coast of Japan. Biosci. Biotechnol. Biochem. 2012, 76, 1229-1232. [CrossRef] [PubMed] 
157. Piersimoni, C.; Scarparo, C. Extrapulmonary infections associated with nontuberculous Mycobacteria in immunocompetent persons. Emerg. Infect. Dis. 2009, 15, 1351-1548. [CrossRef] [PubMed]

158. Duran, N.; Menck, C.F. Chromobacterium violaceum: A review of pharmacological and industiral perspectives. Crit. Rev. Microbiol. 2001, 27, 201-222. [CrossRef] [PubMed]

159. Ryan, R.P.; Monchy, S.; Cardinale, M.; Taghavi, S.; Crossman, L.; Avison, M.B.; Berg, G.; Van Der Lelie, D.; Dow, J.M. The versatility and adaptation of bacteria from the genus Stenotrophomonas. Nat. Rev. Microbiol. 2009, 7, 514-525. [CrossRef] [PubMed]

160. Jeffries, J.L.; Jia, J.; Choi, W.; Choe, S.; Miao, J.; Xu, Y.; Powell, R.; Lin, J.; Kuang, Z.; Gaskins, H.R.; et al. Pseudomonas aeruginosa pyocyanin modulates mucin glycosylation with sialyl-Lewis ${ }^{\mathrm{x}}$ to increase binding to airway epithelial cells. Mucosal Immunol. 2015, 9, 1039-1050. [CrossRef] [PubMed]

161. Kumar, M.; Dwivedi, P.; Sharma, A.K.; Sankar, M.; Patil, R.D.; Singh, N.D. Apoptosis and lipid peroxidation in ochratoxin A- and citrinin-induced nephrotoxicity in rabbits. Toxicol. Ind. Health 2014, 30, 90-98. [CrossRef]

162. Mapari, S.A.S.; Thrane, U.; Meyer, A.S. Fungal polyketide azaphilone pigments as future natural food colorants? Trends Biotechnol. 2014, 28, 300-307. [CrossRef]

163. Frisvad, J.C.; Yilmaz, N.; Thrane, U.; Rasmussen, K.B.; Houbraken, J.; Samson, R.A. Talaromyces atroroseus, a new species efficiently producing industrially relevant red pigments. PLoS ONE 2013, 8, e84102. [CrossRef]

164. Medentsev, A.G.; Akimenko, V.K. Naphthoquinone metabolites of the fungi. Phytochemistry 1998, 47, $935-959$. [CrossRef]

(C) 2019 by the authors. Licensee MDPI, Basel, Switzerland. This article is an open access article distributed under the terms and conditions of the Creative Commons Attribution (CC BY) license (http://creativecommons.org/licenses/by/4.0/). 